Supporting information

\title{
Enecarbamates as Imine Surrogates: Nucleophilic Addition of 1,3-Dicarbonyl Compounds to Enecarbamates
}

Shū Kobayashi,* Tomas Gustafsson, Yusuke Shimizu, Hiroshi Kiyohara, Ryosuke Matsubara

Graduate School of Pharmaceutical Sciences, The University of Tokyo, The HFRE Division, ERATO, Japan Science and Technology Agency (JST), Hongo, Bunkyo-ku, Tokyo, 113-0033, Japan

skobayas@mol.f.u-tokyo.ac.jp

Contents:

S2-S3: $\quad$ General methods and materials

S3-S19: Experimental and physical details for compounds $1 \mathbf{a}-\mathbf{b}$, $2 \mathrm{e}^{-\mathrm{g}}$ and $3 \mathrm{a}^{-\mathrm{z}}$ 
Experimental details and physical data of the products.

General. ${ }^{1} \mathrm{H}$ and ${ }^{13} \mathrm{C}$ NMR spectra were recorded on a JEOL JNM-DE400 or JNM-DE600 spectrometer in $\mathrm{CDCl}_{3}$ unless otherwise noted. Tetramethylsilane (TMS) or residual $\mathrm{CHCl}_{3}$ served as internal standards ( $\delta=0$ and $7.26 \mathrm{ppm}$, respectively) for ${ }^{1} \mathrm{H}$ NMR, and $\mathrm{CDCl}_{3}$ was used as internal standard $(\delta=77.0)$ for ${ }^{13} \mathrm{C}$ NMR. When possible, one of the two diastereomers from compounds $\mathbf{3}$ were partially enriched using normal phase HPLC (Hexane:EtOAc) for assignment of ${ }^{1} \mathrm{H}$ and ${ }^{13} \mathrm{C}$ NMR-signals to the diastereomers. IR spectra were measured with a JASCO FT/IR-610 spectrometer, IR spectra were not measured for mixtures of diastereomers. High-performance liquid chromatography was carried out using following apparatuses; SHIMADZU LC-10AT (liquid chromatograph), SHIMADZU SPD-10A (UV detector), and SHIMADZU C-R6A Chromatopac. Mass spectrometry analysis was carried out using a JEOL The MStation, JMS-700. Column chromatography was conducted on Silica gel 60 (Merck) and preparative thin-layer chromatography was carried out using Wakogel B-5F.

All reactions were carried out under an argon atmosphere in dried glassware. All solvents were dried and distilled using standard procedures. Metal triflates were dried under vacuum prior to use; $\mathrm{Cu}(\mathrm{OTf})_{2}$ at $100{ }^{\circ} \mathrm{C} 6 \mathrm{~h}, \mathrm{Sc}(\mathrm{OTf})_{3}$ at $200{ }^{\circ} \mathrm{C}$ for $72 \mathrm{~h}$ then at $200{ }^{\circ} \mathrm{C}$ for $2 \mathrm{~h}$ just before use, $\mathrm{Yb}(\mathrm{OTf})_{3}$ and $\mathrm{Zn}(\mathrm{OTf})_{2}$ at $200{ }^{\circ} \mathrm{C}$ for $6 \mathrm{~h}$. $\mathrm{Bi}(\mathrm{OTf})_{3}$, $\mathrm{Sn}(\mathrm{OTf})_{2}$ and CuOTf $\cdot 0.5$ toluene were used as commercial grade. (Z)-Encarbamates 1a-c, ${ }^{1}(E)$-enecarbamates $\mathbf{1 d - \mathbf { e } ^ { 2 }}$ and $\beta$-keto esters $\mathbf{2 c},{ }^{3} \mathbf{2 e - g},{ }^{4} \mathbf{2 j}^{5}$ and $\mathbf{2} \mathbf{k}^{6}$ were

\footnotetext{
${ }^{1}$ Mecozzi, T.; Petrini, M. Synlett 2000, 73.Kamatani, A.

2 Kamatani, A.; Overman, L. E. J. Org. Chem. 1999, 64, 8743.

${ }^{3}$ Christoffers, J.; Werner, T.; Unger, S.; Frey, W. Eur. J. Org. Chem. 2003, 425.

4 van Emelen, K.; de Wit, T.; Hoornaert, G.J.; Compernolle, F. Tetrahedron 2002, 58, 4225.

${ }^{5}$ Christoffers, J.; Werner, T.; Frey, W.; Baro, W. Chem. Eur. J. 2004, 10, 1042.
} 
prepared in accordance with literature methods. As enecarbamate 1b, $\beta$-ketoesters $\mathbf{2 e - g}$ and compounds 3a-z are new compounds, they were fully characterized in this report.

$\overbrace{}^{\mathrm{NHCbz}}$

(Z)-(3-Phenyl-propenyl)-carbamic acid benzyl ester (1a): ${ }^{1}{ }^{1} \mathrm{H}$ NMR $\left(\mathrm{CDCl}_{3}, 400\right.$ MHz): $\delta=3.33$ (d, 2H, $J=7.8 \mathrm{~Hz}$ ), 4.88 (dd, 1H, $J=8.2,7.8 \mathrm{~Hz}$ ), 5.18 (s, 2H), 6.50 (d, $1 \mathrm{H}, J=9.6 \mathrm{~Hz}$ ), 6.67 (dd, 1H, $J=9.6,8.2 \mathrm{~Hz}$ ), 7.17-7.26 (m, 4H), 7.27-7.41 (m, 6H); ${ }^{13} \mathrm{C}$ NMR $\left(\mathrm{CDCl}_{3}, 100 \mathrm{MHz}\right): \delta=31.5,67.2,107.3,123.1,126.1,126.2,128.1,128.3$, 128.4, 128.6, 136.8, 129.5, 153.5; HR-MS (ESI) calcd for $\mathrm{C}_{17} \mathrm{H}_{17} \mathrm{NNaO}_{2}{ }^{+} 290.1151$ $[M+\mathrm{Na}]^{+}$, found 290.1160 .<smiles>CC(C)(C)OC(=O)N/C=C\Cc1ccccc1</smiles>

(Z)-(3-Phenyl-propenyl)-carbamic acid tert-butyl ester (1b): ${ }^{1}{ }^{1} \mathrm{NMR}\left(\mathrm{CDCl}_{3}, 400\right.$ MHz, rotamers): $\delta=1.45$ and $1.48(2 \mathrm{~s}, 9 \mathrm{H}), 3.32$ (d, $2 \mathrm{H}, J=7.8 \mathrm{~Hz}), 4.80$ (q, 0.8H, $J=$ $7.8 \mathrm{~Hz}$ ), 5.14 (bs, 0.2H), 6.26 (d, $0.8 \mathrm{H}, J=10.0 \mathrm{~Hz}$ ), 6.42 (bs, 0.2H), 6.57 (t, 1H, J = $10.0 \mathrm{~Hz}), 7.16-7.24(\mathrm{~m}, 3 \mathrm{H}), 7.26-7.33(\mathrm{~m}, 2 \mathrm{H}) ;{ }^{13} \mathrm{C} \mathrm{NMR}\left(\mathrm{CDCl}_{3}, 100 \mathrm{MHz}\right.$, rotamers): $\delta=28.2,31.1,31.5,80.5,106.0,123.3,126.0,126.2,128.1,128.3,128.4$, 128.5, 128.5, 139.9, 152.8; IR (neat) 3351, 3062, 3027, 2978, 2931, 1701, 1674, 1495, 1455, 1366, 1244, 1162, 1057, 861, 741, $699 \mathrm{~cm}^{-1}$; HR-MS (ESI) calcd for $\mathrm{C}_{14} \mathrm{H}_{19} \mathrm{NNaO}_{2}{ }^{+} 256.1308[\mathrm{M}+\mathrm{Na}]^{+}$, found 256.1313 .

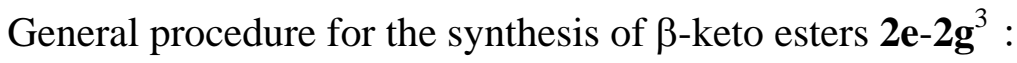<smiles>COC(=O)C1Cc2cc(OC)ccc2C1=O</smiles>

Methyl 5-methoxy-1-indanone-2-carboxylate (2e): 5-Methoxy-1-indanone 1.62 g, 10

\footnotetext{
${ }^{6}$ Dowd, P.; Choi, S-C. Tetrahedron, 1991, 47 (27), 4847.
} 
mmol) in dimethylcarbonate $(10 \mathrm{~mL})$ was added to a suspension of $\mathrm{NaH}$ (880 $\mathrm{mg}, 22$ mmol, $60 \%$ in paraffin) in dimethylcarbonate $(5 \mathrm{~mL})$ and stirred at $90{ }^{\circ} \mathrm{C}$ for $30 \mathrm{~min}$. The resulting solid was dissolved in $\mathrm{H}_{2} \mathrm{O}(20 \mathrm{~mL})$ and $\mathrm{HCl}(2 \mathrm{M}, 5 \mathrm{~mL})$, extracted with $\mathrm{CH}_{2} \mathrm{Cl}_{2} \times 3$ and dried over $\mathrm{Na}_{2} \mathrm{SO}_{4}$. Purification using column chromatography (Hex:EtOAc 3:1) gave a solid that was recrystallized from $i \operatorname{Pr}_{2} \mathrm{O}$ to give $1.68 \mathrm{~g}$ of $\beta$-keto ester 2e (76\%). ${ }^{1} \mathrm{H}$ NMR $\left(\mathrm{CDCl}_{3}, 400 \mathrm{MHz}\right): \delta=3.30$ (dd, $1 \mathrm{H}, J=17.4,8.2 \mathrm{~Hz}$ ), 3.51 (dd, 1H, $J=17.4,4.1 \mathrm{~Hz}$ ), 3.72 (dd, $1 \mathrm{H}, J=8.2,4.1 \mathrm{~Hz}$ ), 3.78 (s, 3H), 3.89 (s, 3H), $6.91(\mathrm{~s}, 1 \mathrm{H}), 6.93$ (d, $1 \mathrm{H}, J=9.1 \mathrm{~Hz}), 7.7$ (d, $1 \mathrm{H}, J=9.1 \mathrm{~Hz}) ;{ }^{13} \mathrm{C} \mathrm{NMR}\left(\mathrm{CDCl}_{3}, 100\right.$ MHz): $\delta=30.2$, 52.7, 53.3, 55.7, 109.5, 115.9, 126.3, 128.3, 156.7, 165.9, 169.8, 197.4; IR (neat) 3394, 2952, 2843, 1740, 1703, 1599, 1436, 1259, 1090, 1025, $649 \mathrm{~cm}^{-1}$; HR-MS (ESI) calcd for $\mathrm{C}_{12} \mathrm{H}_{12} \mathrm{NaO}_{4}{ }^{+} 243.0628[\mathrm{M}+\mathrm{Na}]^{+}$, found 243.0633 .<smiles>COC(=O)C1Cc2cc(Cl)ccc2C1=O</smiles>

Methyl 5-chloro-1-indanone-2-carboxylate (2f): The procedure above gave $1.31 \mathrm{~g}$ $\beta$-keto ester $2 \mathbf{f}$ (58\%) after recrystalization from $i \mathrm{PrOH} .{ }^{1} \mathrm{H} \mathrm{NMR}\left(\mathrm{CDCl}_{3}, 400 \mathrm{MHz}\right): \delta$ $=3.33$ (dd, $1 \mathrm{H}, J=17.9,8.7 \mathrm{~Hz}$ ), 3.51 (dd, $1 \mathrm{H}, 17.9,3.7 \mathrm{~Hz}$ ), 3.73 (dd, $1 \mathrm{H}, J=8.7,3.7$ $\mathrm{Hz}$ ), 3.77 (s, 3H), 7.36 (d, 1H, $J=9.2 \mathrm{~Hz}$ ), 7.48 (s, 1H), 7.66 (d, $1 \mathrm{H}, J=9.2 \mathrm{~Hz}$ ); ${ }^{13} \mathrm{C}$ NMR $\left(\mathrm{CDCl}_{3}, 100 \mathrm{MHz}\right): \delta=29.9,52.9,53.2,125.8,126.7,128.7,133.6,142.1,155.0$, 169.1, 197.8; IR (neat) 3324, 2958, 1715, 1677, 1599, 1509, 1457, 1338, 1266, 1218, 1044, 739, 698, $419 \mathrm{~cm}^{-1}$; HR-MS (ESI) calcd for $\mathrm{C}_{11} \mathrm{H}_{9} \mathrm{ClNaO}_{3}{ }^{+} 247.0132[\mathrm{M}+\mathrm{Na}]^{+}$, found 247.0130 .

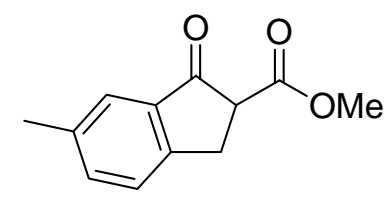

Methyl 6-methyl-1-indanone-2-carboxylate (2g): The procedure above gave $1.20 \mathrm{~g}$ $\beta$-keto ester $2 \mathbf{g}$ (59\%) after recrystalization from iPrOH. ${ }^{1} \mathrm{H}$ NMR $\left(\mathrm{CDCl}_{3}, 400 \mathrm{MHz}\right): \delta$ $=2.41$ (s, 3H), 3.32 (dd, $1 \mathrm{H}, J=16.9,7.8 \mathrm{~Hz}), 3.49$ (dd, $1 \mathrm{H}, J=16.9,4.1 \mathrm{~Hz}$ ), 3.72 (dd, $1 \mathrm{H}, J=7.8,4.1 \mathrm{~Hz}$ ), 3.73 (s, 3H), 7.38 (d, 1H, $J=7.8 \mathrm{~Hz}$ ), 7.50 (dd, $1 \mathrm{H}, J=7.8 \mathrm{~Hz}$ ), 7.57 (s, $1 \mathrm{H}) ;{ }^{13} \mathrm{C} \mathrm{NMR}\left(\mathrm{CDCl}_{3}, 100 \mathrm{MHz}\right): \delta=21.1,30.0,52.8,53.6,121.2,124.7$, 
126.3, 136.8, 138.0, 151.1, 169.8, 199.6; IR (neat) 3290, 3004, 2954, 2921, 2898, 1685, 1626, 1589, 1573, 1441, 138, 1178, 1092, 775, 552, $418 \mathrm{~cm}^{-1}$; HR-MS (ESI) calcd for $\mathrm{C}_{12} \mathrm{H}_{12} \mathrm{NaO}_{3}{ }^{+} 227.0679[M+\mathrm{Na}]^{+}$, found 227.0681 .

General procedure for the synthesis of compounds 3a-z:<smiles>CCOC(=O)C1(C(CBr)NC(=O)OCc2ccccc2)CCCC1=O</smiles>

Benzyl 1-(1-(ethoxycarbonyl)-2-oxocyclopentyl)-3-phenylpropylcarbamate (3a): To a suspension of $\beta$-keto ester 2a (47 mg, $0.3 \mathrm{mmol}$ ) and $\mathrm{Cu}(\mathrm{OTf})_{2}(7.2 \mathrm{mg}, 0.02 \mathrm{mmol})$ in DCE $(1 \mathrm{~mL})$ was added a solution of $1 \mathrm{a}(1 \mathrm{~mL}, 0.2 \mathrm{M}, 0.2 \mathrm{mmol})$ in DCE during 12 h. After $10 \mathrm{~min}$ of additional stirring, the reaction was quenched by the addition of $\mathrm{NaHCO}_{3}$ (aq, sat.), extracted with $\mathrm{CH}_{2} \mathrm{Cl}_{2} \times 2$ and dried over $\mathrm{Na}_{2} \mathrm{SO}_{4}$. Purification by preparative TLC (Hex:EtOAc 4:1) gave $76 \mathrm{mg}$ of product 3a (89\%), isolated as a 1:1.2 mixture of diastereomers. ${ }^{1} \mathrm{H}$ NMR $\left(\mathrm{CDCl}_{3}, 400 \mathrm{MHz}\right)$; diastereomer $1: \delta=1.21(\mathrm{t}, 3 \mathrm{H}$, $J=6.9 \mathrm{~Hz}), 1.68-1.77$ (m, 1H), 1.84-1.95 (m, 3H), 1.95-2.06 (m, 2H), 2.31-2.40 (m, $1 \mathrm{H}), 2.43-2.51(\mathrm{~m}, 1 \mathrm{H}), 2.52-2.61(\mathrm{~m}, 1 \mathrm{H}), 2.72-2.82(\mathrm{~m}, 1 \mathrm{H}), 4.02$ (dt, $1 \mathrm{H}, J=9.6,2.3$ Hz), 4.13 (q, 2H, $J=6.9 \mathrm{~Hz}$ ), 5.06 (d, 1H, $J=11.9 \mathrm{~Hz}$ ), 5.13 (d, 1H, $J=11.9 \mathrm{~Hz}$ ), 5.35 (d, $1 \mathrm{H}, J=11.0 \mathrm{~Hz}), 7.12-7.43(\mathrm{~m}, 10 \mathrm{H})$; diastereomer 2: $\delta=1.18$ (t, $3 \mathrm{H}, J=6.9 \mathrm{~Hz}$ ), 1.59-1.68 (m, 1H), 1.81-1.91 (m, 1H), 1.91-2.02 (m, 2H), 2.05-2.18 (m, 2H), 2.19-2.25 (m, 1H), 2.28-2.36 (m, 1H), 2.39-2.49 (m, 1H), 2.54-2.63 (m, 1H), 2.66-2.76 (m, 1H), 4.04-4.16 (m, 3H), 5.11 (s, 1H), 5.68 (d, 1H, $J=10.5$ Hz), 7.12-7.18 (m, 2H), 7.22-7.28 (m, 2H), 7.28-7.38 (m, 6H); ${ }^{13} \mathrm{C} \mathrm{NMR}\left(\mathrm{CDCl}_{3}, 150 \mathrm{MHz}\right)$, diastereomer $1: \delta=14.0$, 19.0, 32.0, 32.9, 33.7, 37.7, 52.8, 61.6, 64.2, 66.9, 126.0, 128.0, 128.1, 128.4, 128.5, 136.5, 141.3, 156.8, 170.1, 212.1; diastereomer 2: $\delta=14.0$, 19.2, 32.7, 33.1, 33.2, 38.7, 53.1, 61.6, 63.6, 66.8, 125.9, 128.0, 128.1, 128.3, 128.4, 136.5 141.4, 156.5, 171.3, 214.1; HR-MS (ESI) calcd for $\mathrm{C}_{25} \mathrm{H}_{29} \mathrm{NNaO}_{5}{ }^{+} 446.1938[M+\mathrm{Na}]^{+}$, found 446.1934. 
<smiles>CCOC1(C(CBr)CBr)CCCC1=O</smiles>

tert-Butyl 1-(1-(ethoxycarbonyl)-2-oxocyclopentyl)-3-phenylpropylcarbamate (3b): $\beta$-keto ester 2a, $\mathrm{Cu}(\mathrm{OTf})_{2}$ and enecarbamate $\mathbf{1 b}$ were mixed according to the general procedure to give $76 \mathrm{mg}$ of product $\mathbf{3 b}(97 \%)$, isolated as a 1:2 mixture of diastereomers. ${ }^{1} \mathrm{H}$ NMR $\left(\mathrm{CDCl}_{3}, 400 \mathrm{MHz}\right)$; diastereomer 1: $\delta=1.16-1.30(\mathrm{~m}, 5 \mathrm{H}), 1.44(\mathrm{~s}, 9 \mathrm{H})$, 1.54-1.75 (m, 1H), 1.79-2.02 (m, 2H), 2.03-2.16 (m, 1H), 2.22-2.38 (m, 1H), 2.39-2.50 (m, 1H), 2.50-2.62 (m, 1H), 2.67-2.83 (m, 1H), 3.91-4.03 (m, 1H), 4.14 (q, 2H, $J=6.9$ Hz), 5.10 (d, $1 \mathrm{H}, J=10.0 \mathrm{~Hz}$ ), 7.10-7.20 (m, 2H), 7.20-7.31 (m, 3H); diastereomer 2: $\delta$ $=1.18-1.30(\mathrm{~m}, 5 \mathrm{H}), 1.44(\mathrm{~s}, 9 \mathrm{H}), 1.55-1.67(\mathrm{~m}, 1 \mathrm{H}), 1.80-1.91(\mathrm{~m}, 1 \mathrm{H}), 1.91-2.00(\mathrm{~m}$, 1H), 2.04-2.16 (m, 1H), 2.23-2.34 (m, 1H), 2.49-2.62 (m, 1H), 2.65-2.76 (m, 1H), 3.14 (t, $1 \mathrm{H}, J=9.2 \mathrm{~Hz}), 4.00$ (dt, 1H, $J=10.0,3.2 \mathrm{~Hz}), 4.07-4.21(\mathrm{~m}, 2 \mathrm{H}), 5.43$ (d, $1 \mathrm{H}, J=$

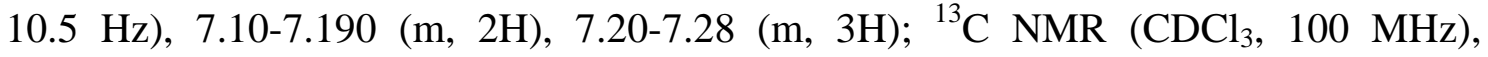
diastereomer 1 (rotamers): $\delta=14.0,14.1,19.2,20.9,27.4,28.3$, 32.8, 33.2, 38.1, 52.3, 54.8, 61.4, 61.5, 63.8, 79.3, 125.8, 128.3, 128.5, 141.7, 155.9 171.5, 214.3; diastereomer 2 (rotamers): $\delta=14.0,14.1,18.9,20.9,28.3,31.9,33.0,33.7,37.7,52.1$, 61.4, 64.4, 79.5, 125.9, 128.4, 128.5, 141.4, 156.2 170.0, 212.1; HR-MS (ESI) calcd for $\mathrm{C}_{22} \mathrm{H}_{32} \mathrm{NO}_{5}{ }^{+} 390.2275[M+\mathrm{H}]^{+}$, found 390.2273 .<smiles>CCOC1(C(N)CC(C)C)CCCC1=O</smiles>

Benzyl 1-(1-(ethoxycarbonyl)-2-oxocyclopentyl)-3-methylbutylcarbamate (3c): $\beta$-keto ester 2a, $\mathrm{Cu}(\mathrm{OTf})_{2}$ and enecarbamate 1c were mixed according to the general procedure to give $52 \mathrm{mg}$ of product 3c (69\%), isolated as a 1:1 mixture of diastereomers. ${ }^{1} \mathrm{H}$ NMR $\left(\mathrm{CDCl}_{3}, 600 \mathrm{MHz}\right)$; both isomers: $\delta=0.88-0.94(\mathrm{~m}, 6 \mathrm{H}), 1.19-1.26(\mathrm{~m}, 3 \mathrm{H})$, 1.63-1.73 (m, 2H), 1.94-2.05 (m, 3H), 2.35-2.40 (m, 2H), 2.51-2.54 (m, 1H), 2.68-2.78 (m, 1H), 4.06-4.19 (m, 3H), 5.04-5.11 (m, 2H), 5.21 and 5.50 (d, 1H, $J=10.3 \mathrm{~Hz}$ ), 7.30-7.35 (m, 5H); ${ }^{13} \mathrm{C} \mathrm{NMR}\left(\mathrm{CDCl}_{3}, 150 \mathrm{MHz}\right) ; \delta=14.1,14.2,19.1,19.3,21.3,21.4$, 23.7, 23.8, 24.9, 25.1, 32.1, 33.1, 37.9, 38.1, 38.8, 40.3, 40.8, 51.5, 61.5, 61.6, 63.9, 64.4, 66.8, 67.1, 127.9, 128.0, 128.1, 128.2, 128.5, 136.6, 136.6, 156.3, 156.7, 170.2, 171.4, 212.3, 214.1; HR-MS (ESI) calcd for $\mathrm{C}_{21} \mathrm{H}_{29} \mathrm{NNaO}_{5}{ }^{+} 398.1938[\mathrm{M}+\mathrm{Na}]^{+}$, found 
398.1921.<smiles>CC(=O)OC(=O)CC(NC(=O)Br)C1(C(C)=O)CCOC1=O</smiles>

Benzyl 1-(3-acetyl-tetrahydro-2-oxofuran-3-yl)-3-phenylpropylcarbamate (3d): $\beta$-keto ester $\mathbf{2 b}, \mathrm{Cu}(\mathrm{OTf})_{2}$ and enecarbamate 1a were mixed according to the general procedure to give $62 \mathrm{mg}$ of product $\mathbf{3 d}$ (78\%), isolated as a single diastereomer. ${ }^{1} \mathrm{H}$ NMR (CDCl $3,400 \mathrm{MHz}): \delta=1.65-1.78(\mathrm{~m}, 1 \mathrm{H}), 1.86-1.98(\mathrm{~m}, 1 \mathrm{H}), 2.19-2.35(\mathrm{~m}, 1 \mathrm{H})$, 2.21 (s, 3H), 2.41-2.64 (m, 1H), 2.69-2.82 (m, 1H), 3.68 (dd, 1H, J = 9.2, $6.9 \mathrm{~Hz}$ ), 4.09-4.19 (m, 1H), 4.27-4.40 (m, 2H), 5.07-5.17 (m, 2H), 5.64 (d, 1H, $J=10.5 \mathrm{~Hz}$ ), 7.11-7.44 (m, 10H); ${ }^{13} \mathrm{C} \mathrm{NMR}\left(\mathrm{CDCl}_{3}, 100 \mathrm{MHz}\right): \delta=26.4,29.0,32.3,33.2,52.4,53.0$, 64.8, 65.6, 67.2, 126.2, 128.0, 128.3, 128.4, 128.5 128.6, 136.1, 140.7, 156.3, 174.7, 203.4; IR (neat) 3404, 2924, 2859, 1763, 1710, 1509, 1455, 1227, 1171, 1105, 1028, 738, $699 \mathrm{~cm}^{-1}$; HR-MS (ESI) calcd for $\mathrm{C}_{23} \mathrm{H}_{25} \mathrm{NNaO}_{5}{ }^{+} 418.1625[\mathrm{M}+\mathrm{Na}]^{+}$, found 418.1616.<smiles>COC(=O)CC(NC(=O)OCc1ccccc1)C1(C(C)=O)CCN(Br)C1=O</smiles>

Benzyl 1-(3-acetyl-1-benzyl-2-oxopyrrolidin-3-yl)-3-phenylpropylcarbamate (3e): $\beta$-keto ester $\mathbf{2 c}, \mathrm{Sc}(\mathrm{OTf})_{3}$ and enecarbamate 1a were mixed in toluene according to the general procedure to give $70 \mathrm{mg}$ of product 3e (72\%), isolated as a single diastereomer. ${ }^{1} \mathrm{H}$ NMR $\left(\mathrm{CDCl}_{3}, 400 \mathrm{MHz}\right): \delta=1.66-1.77$ (m, 1H), 1.88-1.95 (m, 1H), 1.96-2.04 (m, 1H), 2.18 (s, 3H), 2.56-2.66 (m, 1H), 2.71-2.80 (m, 1H), 3.10-3.17 (m, 1H), 4.28 (dt, $1 \mathrm{H}, J=10.9,1.8 \mathrm{~Hz}$ ), 4.39 (d, 1H, $J=14.2 \mathrm{~Hz}$ ), 4.48 (d, 1H, $J=14.2 \mathrm{~Hz}$ ), 5.09 (s, 2H), 5.12 (s, 2H), 6.16 (d, 1H, $J=10.9 \mathrm{~Hz}), 7.13-7.23(\mathrm{~m}, 4 \mathrm{H}), 7.24-7.38$ (m, 9H); ${ }^{13} \mathrm{C} \mathrm{NMR}$ $\left(\mathrm{CDCl}_{3}, 100 \mathrm{MHz}\right): \delta=25.6,26.5,32.4,33.3,43.6,46.9,53.4,65.1,66.8,125.9,127.8$, 127.8, 128.0, 128.1, 128.1, 128.2, 128.4, 128.4, 128.5, 128.8, 135.6, 136.2, 136.5, 141.3, 156.4, 156.6, 172.1, 205.9; IR (neat) 3395, 3029, 2925, 1709, 1677, 1497, 1454, 1336, 1242, 1094, 1045, 1029, 911, 738, $699 \mathrm{~cm}^{-1}$; HR-MS (ESI) calcd for $\mathrm{C}_{30} \mathrm{H}_{32} \mathrm{~N}_{2} \mathrm{NaO}_{4}{ }^{+}$ $507.2254[M+\mathrm{Na}]^{+}$, found 507.2244. 
<smiles>COC(=O)CC(NC(=O)OC(C)(C)C)C1(C(=O)Br)CCN(Br)C1=O</smiles>

tert-Butyl 1-(3-acetyl-1-benzyl-2-oxopyrrolidin-3-yl)-3-phenylpropylcarbamate (3f): $\beta$-keto ester $2 \mathbf{c}, \mathrm{Cu}(\mathrm{OTf})_{2}$ and enecarbamate $\mathbf{1 b}$ were mixed according to the general procedure to give $82 \mathrm{mg}$ of product $\mathbf{3 f}(91 \%)$, isolated as a 1:1 mixture of diastereomers. ${ }^{1} \mathrm{H}$ NMR $\left(\mathrm{CDCl}_{3}, 400 \mathrm{MHz}\right)$, both isomers: $\delta=1.42$ and $1.44(2 \mathrm{~s}, 18 \mathrm{H})$, 1.64-1.80 (m, 2H), 1.84-1.95 (m, 2H), 1.96-2.00 (m, 1H), 2.09-2.17 (m, 1H), 2.22 (s, 3H), 2.36 (s, 3H), 2.51-2.66 (m, 2H), 2.66-2.83 (m, 3H), 3.05 (dt, 1H, $J=9.6,2.7 \mathrm{~Hz}$ ), 3.08-3.18 (m, 4H), 4.21 (t, 1H, $J=10.9$ Hz), 4.36 (t, 1H, $J=14.7 \mathrm{~Hz}), 4.40-4.53$ (m, 4H), 4.57-4.66 (m, 1H), $5.94(\mathrm{~d}, 1 \mathrm{H}, J=10.1 \mathrm{~Hz}), 7.11-7.34(\mathrm{~m}, 20 \mathrm{H}) ;{ }^{13} \mathrm{C}$ NMR $\left(\mathrm{CDCl}_{3}, 100 \mathrm{MHz}\right): \delta=22.1,25.6,26.2,26.3,28.2,28.2,29.6,32.7,33.4,34.2,43.5$, 43.7, 46.8, 46.9, 52.6, 53.2, 65.2, 67.5, 79.3, 79.7, 125.9, 125.9, 127.7, 127.9, 128.0, 128.3, 128.4, 128.7, 135.6, 135.7, 141.3, 141.5, 155.6, 155.8, 170.4, 172.1, 204.6, 206.1; HR-MS (ESI) calcd for $\mathrm{C}_{27} \mathrm{H}_{34} \mathrm{~N}_{2} \mathrm{NaO}_{4}{ }^{+} 473.2411[M+\mathrm{Na}]^{+}$, found 473.2400 .<smiles>COC(=O)NC(CC(C)C)C1(C(=O)OCc2ccccc2)CCN(Br)C1=O</smiles>

Benzyl 1-(3-acetyl-1-benzyl-2-oxopyrrolidin-3-yl)-3-methylbutylcarbamate (3g): $\beta$-keto ester 2c, $\mathrm{Cu}(\mathrm{OTf})_{2}$ and enecarbamate 1c were mixed according to the general procedure with $4 \mathrm{~h}$ of additional stirring to give $54 \mathrm{mg}$ of product $3 \mathrm{~g}(61 \%)$, isolated as a 4:1 mixture of diastereomers. ${ }^{1} \mathrm{H}$ NMR $\left(\mathrm{CDCl}_{3}, 600 \mathrm{MHz}\right)$, diastereomer $1: \delta=0.89$ (d, 3H, $J=6.2 \mathrm{~Hz}$ ), 0.96 (d, 3H, $J=6.2 \mathrm{~Hz}$ ), 1.27-1.43 (m, 2H), 1.67 (br, 1H), 1.99-2.04 (m, 1H), 2.17-2.22 (m, 1H), 2.23 (s, 3H), 3.14-3.22 (m, 2H), 4.28 (td, 1H, $J=2.8,10.3$ $\mathrm{Hz}$ ), 4.45 (d, 1H, $J=13.7 \mathrm{~Hz}$ ), 4.51 (d, 1H, $J=13.7 \mathrm{~Hz}$ ), 5.07 (d, 1H, $J=11.7 \mathrm{~Hz}$ ), 5.11 (d, $1 \mathrm{H}, J=11.7 \mathrm{~Hz}), 5.35$ (d, $1 \mathrm{H}, J=10.3 \mathrm{~Hz}), 7.24-7.36(\mathrm{~m}, 10 \mathrm{H})$; diastereomer 2: $\delta=$ 0.89 (d, 3H, $J=6.2 \mathrm{~Hz}$ ), 1.00 (d, 3H, $J=6.2 \mathrm{~Hz}$ ), 1.12-1.17 (m, 1H), 1.48-1.53 (m, 1H), 1.60-1.65 (m, 1H), 1.89-1.94 (m, 1H), 2.40 (s, 3H), 2.52-2.56 (m, 1H), 3.06-3.15 (m, 2H), 4.37 (d, 1H, $J=14.5 \mathrm{~Hz}$ ), 4.48 (d, 1H, $J=14.5 \mathrm{~Hz}$ ), 4.51 (d, 1H, $J=10.3 \mathrm{~Hz}$ ), 4.65 (td, $1 \mathrm{H}, J=1.4,10.3 \mathrm{~Hz}), 5.04$ (d, $1 \mathrm{H}, J=12.4 \mathrm{~Hz}$ ), 5.07 (d, $1 \mathrm{H}, J=12.4 \mathrm{~Hz}$ ), 7.18-7.36 (m, 10H); ${ }^{13} \mathrm{C} \mathrm{NMR}\left(\mathrm{CDCl}_{3}, 150 \mathrm{MHz}\right)$, diastereomer 1: $\delta=21.5,23.9,24.6$, 25.7, 26.8, 40.4, 43.7, 47.0, 51.9, 65.4, 66.7, 127.9, 128.0, 128.2, 128.5, 128.8, 135.8, 
136.6, 156.3, 172.2, 206.2; diastereomer 2: $\delta=21.3$, 22.5, 23.8, 25.0, 26.7, 41.1, 43.7, 47.1, 52.2, 67.0, 67.6, 127.8, 128.0, 128.1, 128.2, 128.5, 128.8, 135.9, 136.3, 165.2, 170.6, 204.8; HR-MS (ESI) calcd for $\mathrm{C}_{26} \mathrm{H}_{32} \mathrm{~N}_{2} \mathrm{NaO}_{4}{ }^{+} 459.2254[M+\mathrm{Na}]^{+}$, found 459.2249 .

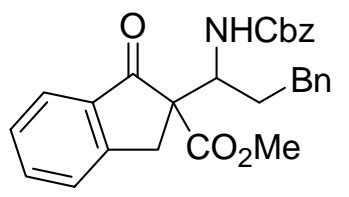

\section{Benzyl 1-(2-(methoxycarbonyl)-2,3-dihydro-1-oxo-1H-inden-2-yl)-3-phenylpropyl-} carbamate (3h); $\beta$-keto ester $\mathbf{2 d}, \mathrm{Sc}(\mathrm{OTf})_{3}$ and enecarbamate 1a were mixed in toluene according to the general procedure to give $72 \mathrm{mg}$ of product $\mathbf{3 h} \mathbf{\mathbf { h }}(79 \%)$, isolated as a 1:1.2 mixture of diastereomers. ${ }^{1} \mathrm{H}$ NMR $\left(\mathrm{CDCl}_{3}, 400 \mathrm{MHz}\right)$; diastereomer $1: \delta=$ 1.64-1.76 (m, 1H), 1.93-2.04 (m, 1H), 2.59-2.65 (m, 1H), 2.68-2.78 (m, 1H), 3.24 (d, $1 \mathrm{H}, J=19.2 \mathrm{~Hz}$ ), 3.50 (d, 1H, $J=19.2 \mathrm{~Hz}$ ), 3.59 (s, 3H), 4.31 (dt, $1 \mathrm{H}, J=10.5,1.8 \mathrm{~Hz}$ ), 5.10-5.20 (m, 2H), 5.86 (d, 1H, $J=10.5$ Hz), 7.10-7.18 (m, 2H), 7.20-2.27 (m, 2H), 7.29-7.46 (m, 8H), 7.61 (dd, 1H, $J=7.8,7.3 \mathrm{~Hz}$ ), 7.75 (d, 1H, $J=7.8 \mathrm{~Hz}$ ); diastereomer 2: $\delta=1.77-1.90(\mathrm{~m}, 1 \mathrm{H}), 1.96-2.09(\mathrm{~m}, 1 \mathrm{H}), 2.56-2.66(\mathrm{~m}, 1 \mathrm{H}), 2.73-2.81(\mathrm{~m}, 1 \mathrm{H}), 3.15$ (d, $1 \mathrm{H}, J=16.5 \mathrm{~Hz}$ ), 3.64-3.76 (m, 1H), 3.66 (s, 3H), 4.21 (dt, $1 \mathrm{H}, J=10.5,2.2 \mathrm{~Hz}$ ), 5.02-5.15 (m, 2H), 5.33 (d, 1H, $J=10.5 \mathrm{~Hz}$ ), 7.11-7.19 (m, 2H), 7.22-2.43 (m, 10H), 7.59 (dd, $1 \mathrm{H}, J=7.3,6.4 \mathrm{~Hz}$ ), 7.73 (d, $1 \mathrm{H}, J=7.3 \mathrm{~Hz}) ;{ }^{13} \mathrm{C} \mathrm{NMR}\left(\mathrm{CDCl}_{3}, 100 \mathrm{MHz}\right)$, diastereomer $1: \delta=32.7,32.9,36.6,52.6,54.6,63.0$, 66.8, 124.7, 125.9, 126.4, 128.0, 128.3, 128.4, 128.5, 135.7, 136.0, 136.5, 141.3, 151.9, 156.4, 171.3, 201.8; diastereomer $2: \delta=32.6$, 34.2, 35.7, 52.8, 55.2, 64.3, 64.3, 124.9, 125.9, 126.2, 127.9, 128.3, 128.4, 135.1, 135.4, 135.6, 135.9, 141.2, 152.2, 156.5, 170.2, 199.4; HR-MS (ESI) calcd for $\mathrm{C}_{28} \mathrm{H}_{27} \mathrm{NNaO}_{5}{ }^{+} 480.1781[\mathrm{M}+\mathrm{Na}]^{+}$, found 480.1776 .<smiles>COC(=O)CC1(C(Cc2ccccc2)NC(=O)OCc2ccccc2)Cc2ccccc2C1=O</smiles>

\section{tert-Butyl 1-(2-(methoxycarbonyl)-2,3-dihydro-1-oxo-1H-inden-2-yl)-3-phenyl-}

propylcarbamate (3i); $\beta$-keto ester $\mathbf{2 d}, \mathrm{Cu}(\mathrm{OTf})_{2}$ and enecarbamate $\mathbf{1 b}$ were mixed according to the general procedure to give $61 \mathrm{mg}$ of product $\mathbf{3 i}(72 \%)$, isolated as a 1:1.5 mixture of diastereomers. ${ }^{1} \mathrm{H}$ NMR $\left(\mathrm{CDCl}_{3}, 400 \mathrm{MHz}\right)$; diastereomer $1: \delta=1.42$ 
(s, 9H), 1.73-1.84 (m, 1H), 1.91-2.02 (m, 1H), 2.54-2.64 (m, 1H), 2.72-2.83 (m, 1H), 3.14 (d, 1H, $J=17.9 \mathrm{~Hz}$ ), 3.69 (d, 1H, $J=17.9 \mathrm{~Hz}$ ), 3.68 (s, 3H), 4.08-4.18 (m, 1H), $5.00(\mathrm{~d}, 1 \mathrm{H}, J=11.0 \mathrm{~Hz}), 7.11-7.18(\mathrm{~m}, 3 \mathrm{H}), 7.20-7.28(\mathrm{~m}, 2 \mathrm{H}), 7.33-7.42(\mathrm{~m}, 2 \mathrm{H})$, $7.58(\mathrm{t}, 1 \mathrm{H}, J=6.9 \mathrm{~Hz}), 7.72(\mathrm{~d}, 1 \mathrm{H}, J=6.9 \mathrm{~Hz})$; diastereomer 2: $\delta=1.45$ (s, 9H), 1.59-1.74 (m, 1H), 1.80-2.00 (m, 1H), 2.52-2.63 (m, 1H), 2.68-2.81 (m, 1H), 3.25 (d, $1 \mathrm{H}, J=17.1 \mathrm{~Hz}$ ), 3.51 (d, 1H, $J=17.1 \mathrm{~Hz}$ ), 3.67 (s, 3H), 4.21 (dt, $1 \mathrm{H}, J=12.4,2.3 \mathrm{~Hz}$ ), 5.61 (d, $1 \mathrm{H}, J=10.5 \mathrm{~Hz}$ ), 7.10-7.31 (m, 5H), 7.35-7.48 (m, 2H), 7.60 (t, $1 \mathrm{H}, J=7.3 \mathrm{~Hz}$ ),

7.75 (d, $1 \mathrm{H}, J=7.3 \mathrm{~Hz}) ;{ }^{13} \mathrm{C} \mathrm{NMR}\left(\mathrm{CDCl}_{3}, 100 \mathrm{MHz}\right)$, diastereomer $1: \delta=28.2,31.5$, 34.3, 35.7, 52.7, 54.8, 64.7, 79.5, 124.7, 125.8, 126.1, 127.9, 128.4, 135.3, 136.0, 141.4 151.1, 155.9, 168.7, 199.6; diastereomer 2: $\delta=28.3$, 32.7, 33.0, 36.7, 52.6, 54.0, 63.1, 79.4, 124.6, 125.9, 126.4, 128.0, 128.3, 128.5, 135.6, 141.5 151.9, 155.8, 171.4, 202.0; HR-MS (ESI) calcd for $\mathrm{C}_{25} \mathrm{H}_{29} \mathrm{NNaO}_{5}{ }^{+} 446.1938[\mathrm{M}+\mathrm{Na}]^{+}$, found 446.1939.<smiles>COC(=O)C1(C(CC(C)C)NC(C)C)Cc2ccccc2C1=O</smiles>

\section{Benzyl 1-(2-(methoxycarbonyl)-2,3-dihydro-1-oxo-1H-inden-2-yl)-3-methylbutyl-} carbamate (3j): $\beta$-keto ester $\mathbf{2 d}, \mathrm{Sc}(\mathrm{OTf})_{3}$ and enecarbamate 1c were mixed according to the general procedure to give $56 \mathrm{mg}$ of product $\mathbf{3} \mathbf{j}(68 \%)$, isolated as a $1: 1$ mixture of diastereomers. ${ }^{1} \mathrm{H}$ NMR $\left(\mathrm{CDCl}_{3}, 400 \mathrm{MHz}\right)$; both isomers: $\delta=0.84$ and $0.88(\mathrm{~d}, 3 \mathrm{H}, J=$ 6.0 and $6.9 \mathrm{~Hz}), 0.92(\mathrm{~d}, 3 \mathrm{H}, J=6.9 \mathrm{H}), 1.21-1.73(\mathrm{~m}, 3 \mathrm{H}), 3.20-3.28(\mathrm{~m}, 1 \mathrm{H})$, 3.52-3.75 (m, 4H), 4.30 and $4.38(\mathrm{t}, 1 \mathrm{H}, J=10.1 \mathrm{~Hz}), 5.04-5.14(\mathrm{~m}, 2 \mathrm{H}), 5.24$ and 5.69 (d, $1 \mathrm{H}, J=6.4$ and 10.6 Hz), 7.31-7.77 (m, 9H); ${ }^{13} \mathrm{C} \mathrm{NMR}\left(\mathrm{CDCl}_{3}, 100 \mathrm{MHz}\right) ; \delta=21.3$, 23.6, 23.7, 24.8, 25.0, 35.8, 36.5, 40.0, 41.2, 52.6, 52.8, 53.0, 53.7, 63.3, 64.5, 66.7, 124.6, 124.8, 126.2, 126.4, 127.8, 127.9, 128.0, 128.4, 135.3, 135.6, 136.1, 136.6, 151.9, 152.2, 156.2, 156.4, 170.4, 171.3, 199.6, 201.9; HR-MS (ESI) calcd for $\mathrm{C}_{24} \mathrm{H}_{27} \mathrm{NNaO}_{5}{ }^{+}$ $432.1781[\mathrm{M}+\mathrm{Na}]^{+}$, found 432.1796 .<smiles>COC(=O)C1(C(NC(=O)c2ccccc2)C(Cc2ccccc2)C(=O)OCc2ccccc2)Cc2ccccc2C1=O</smiles>

Benzyl 1-(2-(methoxycarbonyl)-2,3-dihydro-1-oxo-1H-inden-2-yl)-2-phenylethylcarbamate (3k); $\beta$-keto ester $\mathbf{2 d}, \mathrm{Cu}(\mathrm{OTf})_{2}$ and enecarbamate $\mathbf{1 d}$ were mixed according 
to the general procedure to give $74 \mathrm{mg}$ of product $3 \mathbf{k}(71 \%)$, isolated as a 1:1 mixture of diastereomers. ${ }^{1} \mathrm{H}$ NMR $\left(\mathrm{CDCl}_{3}, 600 \mathrm{MHz}\right.$ ); both diastereomers: $\delta=2.74$ (d, $1 \mathrm{H}, J=$ $11.0 \mathrm{~Hz}$ ), 2.77 (d, 1H, $J=11.0 \mathrm{~Hz}$ ), 2.98-3.04 (m, 1H), 3.19 (dd, $1 \mathrm{H} J=13.7,2.7 \mathrm{~Hz}$ ), 3.37 (d, 1H, $J=17.9 \mathrm{~Hz}$ ), 3.37 (d, 1H, $J=17.1 \mathrm{~Hz}$ ), 3.56 (d, 1H, $J=17.9 \mathrm{~Hz}$ ), 3.63 (s, 3H), 3.68-3.73 (m, 1H), 3.72 (s, 3H), 3.73 (d, $1 \mathrm{H}, J=17.1 \mathrm{~Hz}), 4.48$ (dt, $1 \mathrm{H}, J=10.3$, $4.1 \mathrm{~Hz}$ ), 4.55 (dt, 1H, $J=10.3,4.1 \mathrm{~Hz}$ ), 4.80 (d, 1H, $J=13.1 \mathrm{~Hz}), 4.92$ (d, $1 \mathrm{H}, J=10.3$ $\mathrm{Hz}$ ), 4.94 (d, 1H, $J=11.7 \mathrm{~Hz}$ ), 5.05 (d, 1H, $J=10.3 \mathrm{~Hz}), 5.67$ (d, $1 \mathrm{H}, J=10.3 \mathrm{~Hz}$ ), 7.09-7.32 (m, 18H), 7.37-7.47 (m, 6H), 7.61 (d, 1H, $J=8.3 \mathrm{~Hz}), 7.64$ (d, 1H, $J=8.3$ $\mathrm{Hz}), 7.76$ (d, 1H, 7.6 Hz), 7.81 (d, 1H, J = 7.6 Hz); ${ }^{13} \mathrm{C} \mathrm{NMR}\left(\mathrm{CDCl}_{3}, 150 \mathrm{MHz}\right)$; both diastereomers: $\delta=33.6$, 36.7, 37.3, 37.7, 43.4, 52.7, 52.9, 56.0, 56.7, 63.4, 64.3, 66.5, 124.8, 126.3, 126.5, 126.6, 127.7, 127.9, 128.1, 128.3, 128.4, 129.1, 129.3, 135.5, 135.6, 135.7, 135.8, 136.5, 136.5, 137.6, 137.7, 152. 1, 152.5, 155.9, 156.1, 170.5, 171.3, 200.2, 201.5; HR-MS (ESI) calcd for $\mathrm{C}_{27} \mathrm{H}_{26} \mathrm{NO}_{5}{ }^{+} 444.1806[M+\mathrm{Na}]^{+}$, found 444.1805.<smiles>COC(=O)C1(C(Cc2ccccc2)NC(=O)OCc2ccccc2)Cc2cc(OC)ccc2C1=O</smiles>

\section{Benzyl 1-(2-(methoxycarbonyl)-2,3-dihydro-5-methoxy-1-oxo-1H-inden-2-yl)-3-}

phenylpropylcarbamate (3l); $\beta$-keto ester $\mathbf{2 e}, \mathrm{Cu}(\mathrm{OTf})_{2}$ and enecarbamate $\mathbf{1 a}$ were mixed according to the general procedure to give $74 \mathrm{mg}$ of product $3 \mathbf{l}(76 \%)$, isolated as a 1:1.2 mixture of diastereomers. ${ }^{1} \mathrm{H}$ NMR $\left(\mathrm{CDCl}_{3}, 400 \mathrm{MHz}\right)$; diastereomer $1: \delta=$ 1.75-1.86 (m, 1H), 1.94-2.07 (m, 1H), 2.55-2.65 (m, 1H), 2.71-2.80 (m, 1H), 3.11 (d, $1 \mathrm{H}, J=17.9 \mathrm{~Hz}$ ), 3.63 (d, 1H, $J=17.9 \mathrm{~Hz}$ ), 3.66 (s, 3H), 3.87 (s, 3H), 4.23 (dt, 1H, $J=$ 10.1, $1.8 \mathrm{~Hz}$ ), 5.09 (s, 2H), 5.26 (d, 1H, $J=9.6 \mathrm{~Hz}$ ), 6.80 (s, 1H), 6.89 (d, 1H, $J=8.2$ $\mathrm{Hz}), 7.11-7.38(\mathrm{~m}, 12 \mathrm{H}), 7.65(\mathrm{~d}, 1 \mathrm{H}, J=8.2 \mathrm{~Hz})$; diastereomer $2: \delta=1.62-1.744(\mathrm{~m}$, 1H), 1.88-1.99 (m, 1H), 2.53-2.63 (m, 1H), 2.68-2.77 (m, 1H), 3.16 (d, $1 \mathrm{H}, J=18.3 \mathrm{~Hz}$ ), 3.42 (d, 1H, $J=18.3 \mathrm{~Hz}$ ), 3.59 (s, 3H), 3.88 (s, 3H), 4.28 (dt, 1H, $J=11.0,2.3 \mathrm{~Hz}$ ), 5.09-5.17 (m, 2H), 5.89 (d, 1H, $J=10.1 \mathrm{~Hz}$ ), 6.82 (s, 1H), 6.91 (dd, $1 \mathrm{H}, J=8.2,2.3 \mathrm{~Hz}$ ), 7.10-7.39 (m, 12H), $7.68(\mathrm{~d}, 1 \mathrm{H}, J=8.2 \mathrm{~Hz}) ;{ }^{13} \mathrm{C} \mathrm{NMR}\left(\mathrm{CDCl}_{3}, 150 \mathrm{MHz}\right)$, diastereomer 1: $\delta=32.9$, 34.1, 35.6, 52.8, 55.3, 63.1, 64.6, 109.3, 116.1, 125.9, 126.6, $127.9,128.3,128.4,129.2,141.3,155.3,156.5$, 165.9, 170.5, 197.5; diastereomer $2: \delta=$ 32.9, 36.6, 52.6, 54.8, 55.7, 63.1, 66.8, 109.3, 116.3, 125.9, 126.4, 128.0, 128.3, 128.5, 
136.6, 141.4, 155.0, 156.4, 166.1, 171.5, 199.8; HR-MS (ESI) calcd for $\mathrm{C}_{29} \mathrm{H}_{29} \mathrm{NNaO}_{6}{ }^{+}$ $510.1887[\mathrm{M}+\mathrm{Na}]^{+}$, found 510.1893 .<smiles>COCC1(C(CC(=O)OC)NC(=O)Cc2ccccc2)Cc2cc(OC)ccc2C1=O</smiles>

tert-Butyl 1-(2-(methoxycarbonyl)-2,3-dihydro-1-oxo-1H-inden-2-yl)-3-phenylpropylcarbamate (3m); $\beta$-keto ester $\mathbf{2 e}, \mathrm{Cu}(\mathrm{OTf})_{2}$ and enecarbamate $\mathbf{1 b}$ were mixed according to the general procedure to give $55 \mathrm{mg}$ of product $3 \mathrm{~m}(61 \%)$, isolated as a 1:1 mixture of diastereomers. ${ }^{1} \mathrm{H}$ NMR $\left(\mathrm{CDCl}_{3}, 600 \mathrm{MHz}\right)$; both diastereomers: $\delta=1.43$ and $1.46(\mathrm{~s}, 9 \mathrm{H}), 1.64-1.71$ and 1.75-1.80 (m, 1H), 1.90-1.99 (m, 1H), 2.55-2.63 (m, 1H), 2.71-2.79 (m, 1H), 3.12 and 3.19 (d, 1H, $J=17.2$ and $17.9 \mathrm{~Hz}), 3.44$ and 3.64 (d, $1 \mathrm{H}, J=17.2$ and $17.9 \mathrm{~Hz}$ ), 3.67 and 3.68 (s, 3H), 3.86 (s, 3H), 4.20 (m, $1 \mathrm{H}, J=9.7 \mathrm{~Hz}$ ), 5.01 and 5.67(d, $1 \mathrm{H}, J=11.0$ and $9.7 \mathrm{~Hz}), 6.83-6.91(\mathrm{~m}, 2 \mathrm{H}), 7.12-7.17(\mathrm{~m}, 3 \mathrm{H})$, 7.22-7.25 (m, 2H), 7.63-7.68 (m, 1H); ${ }^{13} \mathrm{C}$ NMR $\left(\mathrm{CDCl}_{3}, 150 \mathrm{MHz}\right)$; both diastereomers: $\delta=28.2$, 28.3, 32.7, 32.9, 34.1, 35.4, 36.6, 52.5, 52.6, 54.0, 54.8, 55.6, 63.2, 64.9, 79.2, 79.4, 109.2, 109.3, 116.0, 116.2, 125.7, 125.8, 126.3, 126.4, 128.2, 128.3, 129.2, 141.4, 141.5, 155.0, 155.2, 155.8, 155.9, 165.7, 165.9, 170.6, 171.6, 197.6, 199.9; HR-MS (ESI) calcd for $\mathrm{C}_{26} \mathrm{H}_{31} \mathrm{NNaO}_{6}{ }^{+} 476.2044[M+\mathrm{Na}]^{+}$, found 476.2034.<smiles>COC(=O)C1(C(CC(C)C)NC(=O)c2ccccc2)Cc2cc(OC)ccc2C1=O</smiles>

\section{Benzyl 1-(2-(methoxycarbonyl)-2,3-dihydro-1-oxo-1H-inden-2-yl)-3-methylbutyl-}

carbamate (3n); $\beta$-keto ester $\mathbf{2 e}, \mathrm{Sc}(\mathrm{OTf})_{3}$ and enecarbamate $\mathbf{1 c}$ were mixed according to the general procedure to give $59 \mathrm{mg}$ of product $\mathbf{3 n}$ (67\%), isolated as a 1:1 mixture of diastereomers. ${ }^{1} \mathrm{H}$ NMR $\left(\mathrm{CDCl}_{3}, 400 \mathrm{MHz}\right.$ ); both diastereomers: 0.85 and 0.88 (d, 3H, $J$ $=6.8$ and $6.4 \mathrm{H}), 0.93$ and $0.94(\mathrm{~d}, 3 \mathrm{H}, J=6.8$ and $6.4 \mathrm{H}), 1.17-1.72(\mathrm{~m}, 3 \mathrm{H}), 3.15-3.21$ (m, 1H), 3.45-3.69 (m, 4H), 3.88 (s, 3H), 4.27-4.37 (m, 1H), 5.04-5.16 (m, 2H), 5.73 (d, $1 \mathrm{H}, J=11.0 \mathrm{~Hz}), 6.87-6.93(\mathrm{~m}, 2 \mathrm{H}), 7.31-7.33(\mathrm{~m}, 5 \mathrm{H}), 7.65-7.71(\mathrm{~m}, 1 \mathrm{H}) ;{ }^{13} \mathrm{C} \mathrm{NMR}$ 
$\left(\mathrm{CDCl}_{3}, 100 \mathrm{MHz}\right) ; \delta=21.3,21.4,23.7,23.8,24.8,25.0,35.3,35.6,40.0,41.0,52.5$, 52.7, 53.1, 53.7, 55.7, 63.4, 64.7, 66.6, 109.3, 109.4, 116.1, 116.2, 126.4, 126.5, 127.8, 127.9, 128.0, 128.4, 128.6, 129.4, 136.7, 139.4, 155.1, 155.4, 156.3, 156.4, 165.9, 166.0, 170.7, 171.5, 197.7, 199.9; HR-MS (ESI) calcd for $\mathrm{C}_{25} \mathrm{H}_{29} \mathrm{NNaO}_{6}{ }^{+} 462.1887[\mathrm{M}+\mathrm{Na}]^{+}$, found 462.1873 .<smiles>COC(=O)C1(C(CBr)NC(=O)OCc2ccccc2)Cc2cc(Cl)ccc2C1=O</smiles>

\section{Benzyl 1-(2-(methoxycarbonyl)-2,3-dihydro-5-chloro-1-oxo-1H-inden-2-yl)-3-}

phenylpropylcarbamate (3o); $\beta$-keto ester $\mathbf{2 f}, \mathrm{Cu}(\mathrm{OTf})_{2}$ and enecarbamate 1a were mixed according to the general procedure to give $94 \mathrm{mg}$ of product $3 \mathbf{0}$ (76\%), isolated as a 1:1 mixture of diastereomers. ${ }^{1} \mathrm{H}$ NMR $\left(\mathrm{CDCl}_{3}, 400 \mathrm{MHz}\right)$; both diastereomers: $\delta=$ 1.65-1.87 (m, 2H), 1.94-2.09 (m, 2H), 2.53-2.65 (m, 2H), 2.69-2.82 (m, 2H), 3.13 (d, $1 \mathrm{H}, J=17.4 \mathrm{~Hz}), 3.24$ (d, $1 \mathrm{H}, J=17.9 \mathrm{~Hz}), 3.47$ (d, $1 \mathrm{H}, J=17.9 \mathrm{~Hz}), 3.59-3.70(\mathrm{~m}$, 7H), 4.21 (dt, $1 \mathrm{H}, J=10.1,1.8 \mathrm{~Hz}), 4.31$ (dt, $1 \mathrm{H}, J=11.0,2.3 \mathrm{~Hz}), 5.06-5.17$ (m, 4H), 5.35 (d, 1H, $J=10.1 \mathrm{~Hz}$ ), 5.79 (d, 1H, $J=10.5 \mathrm{~Hz}$ ), 7.05-7.43 (m, 24H), 7.64 (d, 1H, $J$ $=8.7 \mathrm{~Hz}), 7.67(\mathrm{~d}, 1 \mathrm{H}, J=8.7 \mathrm{~Hz}) ;{ }^{13} \mathrm{C} \mathrm{NMR}\left(\mathrm{CDCl}_{3}, 100 \mathrm{MHz}\right)$; both diastereomers: $\delta$ = 32.6, 32.8, 34.0, 35.4, 36.0, 52.6, 52.8, 54.4, 55.1, 63.4, 64.6, 66.8, 125.7, 125.9, 125.9, 126.0, 126.4, 126.5, 127.9, 128.0, 128.1, 128.3, 128.4, 128.4, 128.7, 128.8, 133.6, 134.3, 136.4, 141.0, 141.1, 142.0, 142.2, 153.3, 153.6, 156.3, 146.5, 169.9, 170.8, 198.1, 200.0; HR-MS (ESI) calcd for $\mathrm{C}_{28} \mathrm{H}_{26} \mathrm{ClNNaO}_{5}{ }^{+} 514.1392[\mathrm{M}+\mathrm{Na}]^{+}$, found 514.1396.<smiles>COC(=O)C1(C(Cc2ccccc2)NC(=O)OCc2ccccc2)C(=O)c2ccc(Cl)cc21</smiles>

tert-Butyl 1-(2-(methoxycarbonyl)-2,3-dihydro-5-chloro-1-oxo-1H-inden-2-yl)-3phenylpropylcarbamate (3p); $\beta$-keto ester $2 \mathbf{f}, \mathrm{Cu}(\mathrm{OTf})_{2}$ and enecarbamate $\mathbf{1 b}$ were mixed according to the general procedure to give $94 \mathrm{mg}$ of product 3p (77\%), isolated as a 1:1.4 mixture of diastereomers. ${ }^{1} \mathrm{H}$ NMR $\left(\mathrm{CDCl}_{3}, 400 \mathrm{MHz}\right)$; both diastereomers: $\delta$ $=1.43(\mathrm{~s}, 9 \mathrm{H}), 1.44$ (s, 9H), 1.59-1.81 (m, 2H), 1.90-2.03 (m, 2H), 2.51-2.64 (m, 2H), 
2.67-2.84 (m, 2H), 3.10 (d, 1H, $J=17.9 \mathrm{~Hz}$ ), 3.23 (d, 1H, $J=17.9 \mathrm{~Hz}$ ), 3.47 (d, $1 \mathrm{H}, J=$ $17.9 \mathrm{~Hz}$ ), 3.62-3.75 (m, 7H), 4.10 (t, 1H, $J=10.1 \mathrm{~Hz}), 4.17$ (t, 1H, $J=10.5 \mathrm{~Hz}), 4.99$ (d, $1 \mathrm{H}, J=11.0 \mathrm{~Hz}$ ), 5.52 (d, 1H, $J=10.1 \mathrm{~Hz}), 7.10-7.29$ (m, 8H), 7.31-7.37 (m, 4H), 7.40 (d, $2 \mathrm{H}, J=13.7 \mathrm{~Hz}), 7.63$ (d, $1 \mathrm{H}, J=7.6 \mathrm{~Hz}), 7.67$ (d, $1 \mathrm{H}, J=7.8 \mathrm{~Hz}) ;{ }^{13} \mathrm{C} \mathrm{NMR}$ $\left(\mathrm{CDCl}_{3}, 100 \mathrm{MHz}\right)$; both diastereomers: $\delta=28.2,28.3,32.7,32.9,34.2,35.4,36.3,52.7$, 53.9, 54.8, 63.5, 64.9, 79.5, 79.7, 125.7, 125.8, 125.9, 126.0, 126.4, 126.6, 128.3, 128.4, 128.5, 128.8, 128.8, 133.7, 141.3, 141.9, 142.2, 153.4, 153.5, 155.9, 170.0, 171.1, 198.1, 200.3; HR-MS (ESI) calcd for $\mathrm{C}_{25} \mathrm{H}_{28} \mathrm{ClNNaO}_{5}{ }^{+} 480.1548[\mathrm{M}+\mathrm{Na}]^{+}$, found 480.1553 .<smiles>COC(=O)C1(C(NC(=O)OCc2ccccc2)C(=O)OCc2ccccc2)Cc2ccc(C)cc2C1=O</smiles>

\section{Benzyl 1-(2-(methoxycarbonyl)-2,3-dihydro-6-methyl-1-oxo-1H-inden-2-yl)-3-}

phenylpropylcarbamate (3q); $\beta$-keto ester $\mathbf{2 g}, \mathrm{Cu}(\mathrm{OTf})_{2}$ and enecarbamate $\mathbf{1 a}$ were mixed according to the general procedure to give $92 \mathrm{mg}$ of product $\mathbf{3 q}$ (98\%), isolated as a 1:1.5 mixture of diastereomers. ${ }^{1} \mathrm{H} \mathrm{NMR}\left(\mathrm{CDCl}_{3}, 400 \mathrm{MHz}\right)$; both diastereomers: $\delta$ $=1.63-1.74(\mathrm{~m}, 1 \mathrm{H}), 1.77-1.89(\mathrm{~m}, 1 \mathrm{H}), 1.89-1.99(\mathrm{~m}, 1 \mathrm{H}), 1.97-2.08(\mathrm{~m}, 1 \mathrm{H}), 2.38(\mathrm{~s}$, $6 \mathrm{H}), 2.53-2.60$ (m, 2H), 2.69-2.82 (m, 2H), 3.11 (d, $1 \mathrm{H}, J=17.4 \mathrm{~Hz}), 3.18$ (d, $1 \mathrm{H}, J=$ $17.9 \mathrm{~Hz}$ ), 3.45 (d, 1H, $J=17.9 \mathrm{~Hz}$ ), 3.59 (s, 3H), 3.61 (d, 1H, $J=17.4 \mathrm{~Hz}$ ), 3.66 (s, 3H), 4.22 (dt, 1H, $J=11.0,2.1 \mathrm{~Hz}$ ), 4.34 (dt, 1H, $J=11.0,2.3 \mathrm{~Hz}$ ), 5.05-5.19 (m, 4H), 5.36 (d, 1H, $J=10.5 \mathrm{~Hz}$ ), 5.90 (d, 1H, $J=10.5 \mathrm{~Hz}$ ), 7.08-7.44 (m, 25H), 7.54 (d, 1H, $J=9.6$ $\mathrm{Hz}) ;{ }^{13} \mathrm{C} \mathrm{NMR}\left(\mathrm{CDCl}_{3}, 100 \mathrm{MHz}\right)$; both diastereomers: $\delta=21.0,21.0,32.6,32.9,34.2$, 35.3, 36.2, 52.5, 52.7, 54.6, 55.2, 63.2, 64.6, 66.7, 124.4, 124.7, 125.8, 126.0, 127.9, 128.0, 128.2, 128.3, 128.4, 135.2, 136.1, 136.5, 136.7, 136.9, 137.7, 138.1, 141.2, 141.3, 149.3, 149.6, 156.4, 156.5, 170.3, 171.3, 199.5, 201.9; HR-MS (ESI) calcd for $\mathrm{C}_{29} \mathrm{H}_{30} \mathrm{NO}_{5}{ }^{+} 472.2119[M+\mathrm{Na}]^{+}$, found 472.2115 .<smiles>COC(=O)C1(C(Cc2ccccc2)NC(C)(C)C)Cc2ccc(C)cc2C1=O</smiles>

tert-Butyl 1-(2-(methoxycarbonyl)-2,3-dihydro-6-methyl-1-oxo-1H-inden-2-yl)-3phenylpropylcarbamate (3r): $\beta$-keto ester $\mathbf{2 g}$, Cu(OTf) $)_{2}$ and enecarbamate $\mathbf{1 b}$ were mixed according to the general procedure to give $86 \mathrm{mg}$ of product $3 \mathbf{r}$ (86\%), isolated 
as a 1:1.6 mixture of diastereomers. ${ }^{1} \mathrm{H}$ NMR $\left(\mathrm{CDCl}_{3}, 400 \mathrm{MHz}\right)$; diastereomer $1: \delta=$ 1.43 (s, 9H), 1.76-1.83 (m, 1H), 1.90-2.04 (m, 1H), 2.37 (s, 3H), 2.32-2.64 (m, 1H), 2.71-2.82 (m, 1H), 3.07 (d, 1H, $J=16.9 \mathrm{~Hz}$ ), 3.64 (d, 1H, $J=16.9 \mathrm{~Hz}$ ), 3.87 (s, 3H), 4.12 (dt, 1H, $J=11.0,1.4 \mathrm{~Hz}$ ), 5.02 (d, 1H, $J=10.1 \mathrm{~Hz}$ ), 7.11-7.19 (m, 2H), 7.21-7.31 $(\mathrm{m}, 4 \mathrm{H}), 7.39$ (d, 1H, $J=7.8 \mathrm{~Hz}), 7.50(\mathrm{~s}, 1 \mathrm{H})$; diastereomer 2: $\delta=1.45(\mathrm{~s}, 9 \mathrm{H})$, 1.59-1.67 (m, 1H), 1.84-1.96 (m, 1H), 2.38 (s, 3H), 2.51-2.61 (m, 1H), 2.67-2.78 (m, 1H), 3.18 (d, 1H, $J=17.9 \mathrm{~Hz}$ ), 3.44 (d, $1 \mathrm{H}, J=17.9 \mathrm{~Hz}$ ), 3.69 (s, 3H), 4.21 (dt, $1 \mathrm{H}, J=$ 11.0, $2.7 \mathrm{~Hz}$ ), 5.64 (d, 1H, $J=10.5 \mathrm{~Hz}$ ), 7.10-7.17 (m, 2H), 7.19-7.27 (m, 3H), 7.30 (d, $1 \mathrm{H}, J=8.2 \mathrm{~Hz}), 7.41$ (d, $1 \mathrm{H}, J=8.2 \mathrm{~Hz}), 7.54$ (s, $1 \mathrm{H}) ;{ }^{13} \mathrm{C} \mathrm{NMR}\left(\mathrm{CDCl}_{3}, 100 \mathrm{MHz}\right)$, diastereomer $1: \delta=28.2$, 32.7, 33.0, 34.3, 35.3, 52.7, 54.8, 64.9, 79.5, 124.6, 125.9, 128.3, 128.4, 135.4, 136.3, 136.6, 137.9, 141.4, 149.5, 155.9, 170.5, 199.6; diastereomer 2: $\delta=21.0,28.3$, 32.7, 33.0, 36.4, 52.6, 54.0, 63.4, 79.3, 124.4, 125.8, 126.0, 128.3, 128.5, 136.3, 136.9, 138.0, 141.6, 149.3, 155.8, 171.6, 202.2; HR-MS (ESI) calcd for $\mathrm{C}_{26} \mathrm{H}_{31} \mathrm{NNaO}_{5}{ }^{+} 460.2094[\mathrm{M}+\mathrm{Na}]^{+}$, found 460.2094 .

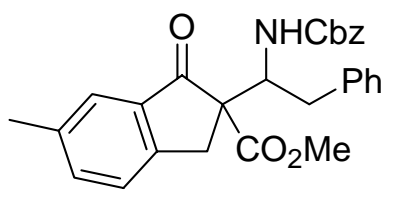

\section{Benzyl 1-(2-(methoxycarbonyl)-2,3-dihydro-6-methyl-1-oxo-1H-inden-2-yl)-2-}

phenylethylcarbamate (3s): $\beta$-keto ester $\mathbf{2 g}, \mathrm{Cu}(\mathrm{OTf})_{2}$ and enecarbamate $\mathbf{1 d}$ were mixed according to the general procedure to give $75 \mathrm{mg}$ of product $3 \mathrm{~s}$ (80\%), isolated as a 1:1.5 mixture of diastereomers. ${ }^{1} \mathrm{H} \mathrm{NMR}\left(\mathrm{CDCl}_{3}, 400 \mathrm{MHz}\right)$; diastereomer $1: \delta=2.92$ (s, 3H), 2.94-3.04 (m, 2H), 3.35 (d, 1H, $J=17.4 \mathrm{~Hz}$ ), 3.67 (d, 1H, $J=17.4 \mathrm{~Hz}$ ), 3.71 (s, 3H), 4.49-4.59 (m, 1H), 4.85 (d, 1H, $J=12.8 \mathrm{~Hz}$ ), 4.93 (d, 1H, $J=12.8 \mathrm{~Hz}$ ), 5.03 (d, $1 \mathrm{H}, J=10.5 \mathrm{~Hz}$ ), 7.08-7.31 (m, 10H), 7.34 (d, 1H, $J=7.8 \mathrm{~Hz}$ ), 7.44 (d, 1H, $J=7.8 \mathrm{~Hz}$ ), 7.55 (s, 1H); diastereomer 2: $\delta=2.42$ (s, 3H), 2.73 (dd, 1H, $J=14.7,11.0 \mathrm{~Hz}$ ), 3.17 (d, $1 \mathrm{H}, J=14.7 \mathrm{~Hz}$ ), 3.31 (d, 1H, $J=17.9 \mathrm{~Hz}$ ), 3.50 (d, 1H, $J=17.9 \mathrm{~Hz}$ ), 3.63 (s, 3H), 4.47 (dt, 1H, $J=11.0,2.4 \mathrm{~Hz}$ ), 4.86 (d, 1H, $J=12.8 \mathrm{~Hz}$ ), 4.93 (d, 1H, $J=12.8 \mathrm{~Hz}$ ), 5.68 (d, $1 \mathrm{H}, J=11.0 \mathrm{~Hz}$ ), 7.11-7.23 (m, 6H), 7.25-7.32 (m, 4H), 7.32 (d, 1H, $J=7.3 \mathrm{~Hz}$ ), 7.45 $(\mathrm{d}, 1 \mathrm{H}, J=7.3 \mathrm{~Hz}), 7.60(\mathrm{~s}, 1 \mathrm{H}) ;{ }^{13} \mathrm{C} \mathrm{NMR}\left(\mathrm{CDCl}_{3}, 100 \mathrm{MHz}\right)$, both diastereomers: $\delta=$ 21.1, 21.1, 35.7, 36.4, 37.3, 37.7, 52.7, 52.8, 56.0, 63.6, 64.6, 66.4, 124.7, 125.9, 126.1, 126.4, 126.5, 127.6, 127.8, 128.3, 129.1, 129.3, 135.6, 136.0, 136.5, 136.9, 137.0, 137.7, 137.7, 138.1, 149.5, 149.9, 155.8, 156.2, 170.7, 171.4, 201.4, 201.5; HR-MS (ESI) 
calcd for $\mathrm{C}_{28} \mathrm{H}_{27} \mathrm{NNaO}_{5}{ }^{+} 480.1781[M+\mathrm{Na}]^{+}$, found 480.1781 .<smiles>CCOC(=O)CC(NC(Br)Br)C1(CBr)CCCCC1=O</smiles>

Benzyl 1-(1-(ethoxycarbonyl)-2-oxocyclohexyl)-3-phenylpropylcarbamate (3t): $\beta$-keto ester $\mathbf{2 h}, \mathrm{Sc}(\mathrm{OTf})_{3}$ and enecarbamate 1a were mixed in toluene according to the general procedure to give $52 \mathrm{mg}$ of product 3t (61\%), isolated as a 1:9 mixture of diastereomers. ${ }^{1} \mathrm{H}$ NMR $\left(\mathrm{CDCl}_{3}, 400 \mathrm{MHz}\right)$; both diastereomers: $\delta=1.21$ (t, 3H, $J=7.1$ Hz), 1.43-1.56 (m, 1H), 1.60-1.75 (m, 2H), 1.76-1.98 (m, 2H), 1.98-2.11 (m, 1H), 2.32-2.40 (m, 1H), 2.43-2.61 (m, 3H), 2.68-2.78 (m, 1H), 4.07-4.20 (m, 3H), 5.05-5.16 (m, 3H), 5.47 (d, 1H, J = 11.0 Hz), 7.12-7.39 (m, 10H); ${ }^{13} \mathrm{C} \mathrm{NMR}\left(\mathrm{CDCl}_{3}, 100 \mathrm{MHz}\right)$; both diastereomers: $\delta=14.0,21.6,22.2,26.6,27.3,32.8,33.0,34.0,34.1,34.3,40.8$, 41.2, 54.2, 61.5, 61.6, 64.8, 66.0, 66.7, 66.8, 125.9, 126.7, 127.9, 128.1, 128.3, 128.5, 128.7, 129.0, 136.5, 141.4, 141.6, 156.3, 156.7, 171.3, 171.5, 208.3, 208.9; HR-MS (ESI) calcd for $\mathrm{C}_{26} \mathrm{H}_{31} \mathrm{NNaO}_{5}{ }^{+} 460.2094[\mathrm{M}+\mathrm{Na}]^{+}$, found 460.2097 .<smiles>COC(=O)CC1(C(Br)Br)CCc2ccccc2C1=O</smiles>

Benzyl 1-(2-(methoxycarbonyl)-1,2,3,4-tetrahydro-1-oxonaphthalen-2-yl)-3-

phenylpropylcarbamate (3u): $\beta$-keto ester $\mathbf{2 i}$, $\mathrm{Cu}(\mathrm{OTf})_{2}$ and enecarbamate $\mathbf{1 a}$ were mixed according to the general procedure to give $67 \mathrm{mg}$ of product $3 \mathbf{u}(71 \%)$, isolated as a 1:1.1 mixture of diastereomers. ${ }^{1} \mathrm{H}$ NMR $\left(\mathrm{CDCl}_{3}, 400 \mathrm{MHz}\right)$; diastereomer $1: \delta=$ 1.71-1.80 (m, 1H), 2.24-2.39 (m, 2H), 2.51-2.65 (m, 2H), 2.72-2.96 (m, 3H), 3.61 (s, 3H), 4.24 (dt, 1H, $J=11.0,2.3 \mathrm{~Hz}$ ), 5.03 (d, 1H, $J=12.0 \mathrm{~Hz}$ ), 5.08 (d, 1H, $J=12.0 \mathrm{~Hz}$ ), 5.29 (d, 1H, $J=10.5 \mathrm{~Hz}$ ), 7.13-7.20 (m, 4H), 7.22-7.35 (m, 8H), 7.45 (dd, 1H, $J=8.2$, $7.8 \mathrm{~Hz}$ ), 7.95 (d, 1H, $J=8.2 \mathrm{~Hz}$ ); diastereomer 2: $\delta=1.83-1.93(\mathrm{~m}, 2 \mathrm{H}), 2.18-2.29(\mathrm{~m}$, $1 \mathrm{H}), 2.41-2.49(\mathrm{~m}, 1 \mathrm{H}), 2.56-2.65(\mathrm{~m}, 1 \mathrm{H}), 2.73-2.83(\mathrm{~m}, 1 \mathrm{H}), 2.83-2.95(\mathrm{~m}, 1 \mathrm{H})$, 2.95-3.04 (m, 1H), 3.55 (s, 3H), 4.18-4.25 (m, 1H), 5.09 (d, 1H, $J=12.4$ Hz), 5.17 (d, $1 \mathrm{H}, J=12.4 \mathrm{~Hz}), 6.09$ (d, 1H, $J=10.5 \mathrm{~Hz}), 7.10-7.41(\mathrm{~m}, 12 \mathrm{H}), 7.46$ (t, $1 \mathrm{H}, J=6.9 \mathrm{~Hz})$, 8.02 (d, $1 \mathrm{H}, J=8.2 \mathrm{~Hz}) ;{ }^{13} \mathrm{C} \mathrm{NMR}\left(\mathrm{CDCl}_{3}, 100 \mathrm{MHz}\right)$, diastereomer $1: \delta=25.5,30.7$, 33.1, 34.7, 52.4, 54.8, 62.6, 66.8, 125.9, 126.8, 127.8, 128.0, 128.3, 128.4, 128.4, 128.5, 
128.6, 128.7, 132.5, 133.6, 136.4, 141.4, 142.3, 156.5, 171.2, 195.2; diastereomer 2: $\delta=$ 26.4, 31.0, 32.7, 33.4, 52.5, 55.3, 62.0, 66.6, 125.8, 126.9, 127.9, 128.0, 128.2, 128.3, 128.4, 128.4, 128.7, 132.2, 133.9, 136.7, 141.5, 142.8, 156.2, 171.5, 195.7; HR-MS (ESI) calcd for $\mathrm{C}_{29} \mathrm{H}_{29} \mathrm{NNaO}_{5}{ }^{+} 494.1938[\mathrm{M}+\mathrm{Na}]^{+}$, found 494.1936 .<smiles>COC(=O)CC(NC(=O)Br)C1(C(C)=O)CC(OC)=CC1=O</smiles>

\section{Benzyl 1-(1-(methoxycarbonyl)-4-methoxy-2-oxocyclopent-3-enyl)-3-phenyl-}

propylcarbamate (3v): $\beta$-keto ester $\mathbf{2 j}$, $\mathrm{Cu}(\mathrm{OTf})_{2}$ and enecarbamate $\mathbf{1 a}$ were mixed according to the general procedure to give $56 \mathrm{mg}$ of product $3 \mathbf{v}$ (64\%), isolated as a 1:1 mixture of diastereomers. ${ }^{1} \mathrm{H}$ NMR $\left(\mathrm{CDCl}_{3}, 400 \mathrm{MHz}\right)$; diastereomer $1: \delta=1.67-1.77$ (m, 1H), 1.87-1.99 (m, 1H), 2.52-2.64 (m, 1H), 2.54 (d, 1H, J =17.9 Hz), 2.70-2.80 (m, 1H), 3.18 (d, 1H, $J=17.9 \mathrm{~Hz}$ ), 3.69 (s, 3H), 3.83 (s, 3H), 4.13 (dt, $1 \mathrm{H}, J=10.1,2.3 \mathrm{~Hz}$ ), 5.09 (d, 1H, $J=12.4 \mathrm{~Hz}$ ), 5.13 (d, 1H, $J=12.4 \mathrm{~Hz}$ ), 5.23 (s, 1H), 5.30 (d, 1H, $J=10.1$ $\mathrm{Hz}), 7.11-7.19(\mathrm{~m}, 4 \mathrm{H}), 7.22-7.40(\mathrm{~m}, 6 \mathrm{H})$; diastereomer $2: \delta=1.60-1.78(\mathrm{~m}, 1 \mathrm{H})$, 1.88-1.99 (m, 1H), 2.49-2.65 (m, 1H), 2.67 (d, 1H, $J=18.3$ Hz), 2.67-2.78 (m, 1H), 2.91 (d, 1H, $J=18.3 \mathrm{~Hz}$ ), 3.61 (s, 3H), 3.83 (s, 3H), 4.20 (dt, 1H, $J=11.0,2.3 \mathrm{~Hz}$ ), 5.10 (d, $1 \mathrm{H}, J=12.8 \mathrm{~Hz}$ ), 5.13 (d, 1H, $J=12.8 \mathrm{~Hz}$ ), 5.28 (s, 1H), 5.81 (d, $1 \mathrm{H}, J=10.5 \mathrm{~Hz}$ ), 7.12-7.20 (m, 4H), 7.22-7.36 (m, 6H); ${ }^{13} \mathrm{C} \mathrm{NMR}\left(\mathrm{CDCl}_{3}, 100 \mathrm{MHz}\right)$, diastereomer 1: $\delta$ = 32.9, 34.0, 37.0, 52.8, 55.1, 59.1, 62.9, 66.8, 102.5, 126.0, 128.0, 128.4, 128.5, 136.6, 141.3, 156.6, 170.0, 189.3, 197.9; diastereomer 2: $\delta=32.8$, 33.9, 37.8, 52.6, 54.3, 59.0, 61.5, 62.9, 104.0, 125.9, 128.1, 128.3, 128.5, 136.6, 141.4, 156.4, 171.0, 189.1, 200.3; HR-MS (ESI) calcd for $\mathrm{C}_{25} \mathrm{H}_{27} \mathrm{NNaO}_{6}{ }^{+} 460.1731[\mathrm{M}+\mathrm{Na}]^{+}$, found 460.1726 .<smiles>COC(=O)CC(NC(=O)OCc1ccccc1)C1(C(=O)OCc2ccccc2)C=CC(=O)C1</smiles>

\section{tert-Butyl 1-(1-(methoxycarbonyl)-4-methoxy-2-oxocyclopent-3-enyl)-3-phenyl-}

propylcarbamate (3w): $\beta$-keto ester $2 \mathbf{j}$, $\mathrm{Cu}(\mathrm{OTf})_{2}$ and enecarbamate $\mathbf{1 b}$ were mixed according to the general procedure to give $60 \mathrm{mg}$ of product $3 \mathbf{w}(74 \%)$, isolated as a 1:1.2 mixture of diastereomers. ${ }^{1} \mathrm{H}$ NMR $\left(\mathrm{CDCl}_{3}, 400 \mathrm{MHz}\right)$; diastereomer $1: \delta=1.44$ (s, 9H),1.78-1.96 (m, 2H), 2.51-2.64 (m, 1H), 2.55 (d, 1H, J = 18.3 Hz), 2.65-2.74 (m, 
1H), 3.16 (d, 1H, $J=18.3 \mathrm{~Hz}$ ), 3.69 (s, 3H), 3.82 (s, 3H), 4.08 (t, 1H, $J=10.5 \mathrm{~Hz}$ ), 5.22, (s, 1H), 5.57 (d, 1H, $J=9.6 \mathrm{~Hz}$ ), 7.11-7.19 (m, 3H), 7.19-7.27 (m, 2H); diastereomer 2: $\delta=1.45$ (s, 9H), 1.81-2.00 (m, 2H), 2.49-2.62 (m, 1H), 2.70 (d, $1 \mathrm{H}, J=18.3 \mathrm{~Hz})$, 2.70-2.80 (m, 1H), 2.91 (d, 1H, $J=18.3 \mathrm{~Hz}$ ), 3.70 (s, 3H), 3.80-3.85 (m, 1H), 3.82 (s, 3H), 5.01 (d, $1 \mathrm{H}, J=12.8 \mathrm{~Hz}$ ), 5.28 (s, 1H), 7.11-7.19 (m, 3H), 7.21-7.29 (m, 2H); ${ }^{13} \mathrm{C}$ NMR $\left(\mathrm{CDCl}_{3}, 100 \mathrm{MHz}\right)$, diastereomer 1: $\delta=28.3$, 32.7, 32.9, 34.0, 37.9, 52.7, 53.7, 59.0, 63.0, 79.5, 104.0, 125.8, 128.3, 141.4, 155.8, 170.2, 189.1, 198.0; diastereomer 2: $\delta=28.3$, 32.8, 32.9, 34.0, 37.0, 52.6, 54.5, 59.0, 61.7, 79.3, 102.5, 125.9, 128.4, 141.6, 155.9, 171.2, 189.1, 200.5; HR-MS (ESI) calcd for $\mathrm{C}_{22} \mathrm{H}_{29} \mathrm{NNaO}_{6}{ }^{+} 426.1887[\mathrm{M}+\mathrm{Na}]^{+}$, found 426.1892 .<smiles>COC(=O)C(CBr)NC(CBr)C1(C(=O)OCc2ccccc2)COCC1=O</smiles>

\section{Benzyl 1-(3-(methoxycarbonyl)-tetrahydro-4-oxofuran-3-yl)-3-phenylpropyl-}

carbamate (3x): $\beta$-keto ester $\mathbf{2 k}, \mathrm{Cu}(\mathrm{OTf})_{2}$ and enecarbamate $\mathbf{1 a}$ were mixed according to the general procedure to give $50 \mathrm{mg}$ of product $3 \mathbf{x}(61 \%)$, isolated as a 1:1.2 mixture of diastereomers. ${ }^{1} \mathrm{H}$ NMR $\left(\mathrm{CDCl}_{3}, 400 \mathrm{MHz}\right)$; diastereomer $1: \delta=1.70-1.80(\mathrm{~m}, 1 \mathrm{H})$, 1.92-2.04 (m, 1H), 2.54-2.65 (m, 1H), 2.75-2.83 (m, 1H), 3.72 (s, 3H), 3.99 (d, 1H, $J=$ $17.4 \mathrm{~Hz}$ ), 4.14 (d, 1H, $J=17.4 \mathrm{~Hz}$ ), 4.13 (d, 1H, $J=9.6 \mathrm{~Hz}$ ), 4.21 (dt, $1 \mathrm{H}, J=11.5,2.4$ Hz), 4.54 (d, 1H, $J=9.6 \mathrm{~Hz}$ ), 5.06 (d, 1H, $J=12.4 \mathrm{~Hz}$ ), 5.12 (d, 1H $J=12.4 \mathrm{~Hz}$ ), 5.37 $(\mathrm{d}, 1 \mathrm{H}, J=10.5 \mathrm{~Hz}), 7.12-7.41(\mathrm{~m}, 10 \mathrm{H})$; diastereomer $2: \delta=2.17-2.29(\mathrm{~m}, 1 \mathrm{H})$, 2.32-2.39 (m, 1H), 2.58-2.66 (m, 1H), 2.66-2.75 (m, 1H), 3.69 (s, 3H), 3.91 (d, 1H, $J=$ $16.9 \mathrm{~Hz}$ ), 4.05-4.16 (m, 2H), 4.19 (dt, 1H, $J=10.5,2.3 \mathrm{~Hz}$ ), 4.44 (d, 1H, $J=10.0 \mathrm{~Hz}$ ), 5.09 (d, 1H, $J=12.0 \mathrm{~Hz}$ ), 5.13 (d, $1 \mathrm{H} J=12.0 \mathrm{~Hz}$ ), 5.52 (d, $1 \mathrm{H}, J=10.5 \mathrm{~Hz}$ ), 7.12-7.35 (m, 10H); ${ }^{13} \mathrm{C} \mathrm{NMR}\left(\mathrm{CDCl}_{3}, 150 \mathrm{MHz}\right)$, diastereomer 1: $\delta=33.0,33.6,52.0,53.1,63.7$, 67.1, 71.2, 73.2, 126.3, 128.1, 128.3, 128.5, 128.6, 136.3, 140.9, 156.7, 168.3, 207.5; diastereomer 2: $\delta=29.3$, 32.8, 33.5, 52.0, 63.6, 67.0, 71.0, 73.0, 126.3, 128.0, 128.1, 128.1, 128.4, 136.2, 140.8, 156.6, 168.1, 201.5; HR-MS (ESI) calcd for $\mathrm{C}_{23} \mathrm{H}_{25} \mathrm{NNaO}_{6}{ }^{+}$ $434.1574[\mathrm{M}+\mathrm{Na}]^{+}$, found 434.1568 . 
<smiles>CC(=O)C(CBr)C(CBr)NC(=O)OCc1ccccc1</smiles>

Benzyl 4-acetyl-5-oxo-1-phenylhexan-3-ylcarbamate (3y): $\beta$-keto ketone 2l, $\mathrm{Cu}(\mathrm{OTf})_{2}$ and enecarbamate 1a were mixed according to the general procedure to give $40 \mathrm{mg}$ of product 3y (55\%). ${ }^{1} \mathrm{H}$ NMR $\left(\mathrm{CDCl}_{3}, 600 \mathrm{MHz}\right): \delta=1.70-1.76(\mathrm{~m}, 1 \mathrm{H})$, 1.94-2.00 (m, 1H), 2.09 (s, 3H), 2.23 (s, 3H), 2.58-2.63 (m, 1H), 2.70-2.85 (m, 1H), 3.88 (d, 1H, $J=4.8 \mathrm{~Hz}$ ), 4.36 (m, 1H d, 1H), 5.05 (d, 1H, $J=12.4 \mathrm{~Hz}$ ), 7.15-7.37 (m, $10 \mathrm{H}) ;{ }^{13} \mathrm{C} \mathrm{NMR}\left(\mathrm{CDCl}_{3}, 150 \mathrm{MHz}\right) ; \delta=29.9,30.9,32.7,35.6,50.6,66.9,69.4,126.2$, 127.9, 128.1, 128.4, 128.6, 136.4, 140.9, 156.2, 203.5, 204.9; HR-MS (ESI) calcd for $\mathrm{C}_{22} \mathrm{H}_{25} \mathrm{NNaO}_{4}{ }^{+} 390.1676[\mathrm{M}+\mathrm{Na}]^{+}$, found 390.1698 .<smiles>CC(=O)C(CC(=O)OC(C)(Br)Br)C(Cc1ccccc1)C(=O)c1ccccc1</smiles>

Benzyl 2-acetyl-1-oxo-1,5-diphenylpentan-3-ylcarbamate (3z): $\beta$-keto ketone 2m, $\mathrm{Cu}(\mathrm{OTf})_{2}$ and enecarbamate 1a were mixed according to the general procedure with $7 \mathrm{~h}$ of additional stirring to give $37 \mathrm{mg}$ of product $\mathbf{3 z}(43 \%)$, isolated as a 1:1 mixture of diastereomers. ${ }^{1} \mathrm{H}$ NMR $\left(\mathrm{CDCl}_{3}, 600 \mathrm{MHz}\right)$; both isomers: $\delta=1.73-1.78$ and 1.87-1.96 $(\mathrm{m}, 1 \mathrm{H}), 1.83-1.88$ and 2.07-2.14 $(\mathrm{m}, 1 \mathrm{H}), 2.10$ and $2.23(\mathrm{~s}, 3 \mathrm{H}), 2.55-2.60$ and 2.62-2.67 (m, 1H), 2.70- $2.75(\mathrm{~m}, 1 \mathrm{H}), 4.41-4.46$ and 4.47-4.52 (m, $1 \mathrm{H}), 4.77$ and 4.79 (d, $1 \mathrm{H}, J=6.9$ and $4.1 \mathrm{~Hz}$ ), 5.05-5.13 (m, 2H), 5.29 and 5.91 (d, $1 \mathrm{H}, J=9.6$ and 10.3 $\mathrm{Hz})$, 7.09-7.88 (m, 15H); ${ }^{13} \mathrm{C} \mathrm{NMR}\left(\mathrm{CDCl}_{3}, 150 \mathrm{MHz}\right) ; \delta=29.1$, 29.7, 32.6, 32.8, 34.9, 35.3, 51.4, 63.7, 65.7, 66.8, 126.1, 126.1, 127.9, 127.9, 128.1, 128.3, 128.4, 128.5, 128.6, 129.0, 134.0, 136.4, 136.5, 140.9, 140.9, 156.0, 156.3, 194.9, 196.9, 203.2, 203.6; HR-MS (ESI) calcd for $\mathrm{C}_{27} \mathrm{H}_{27} \mathrm{NNaO}_{4}{ }^{+} 452.1832[M+\mathrm{Na}]^{+}$, found 452.1832 . 


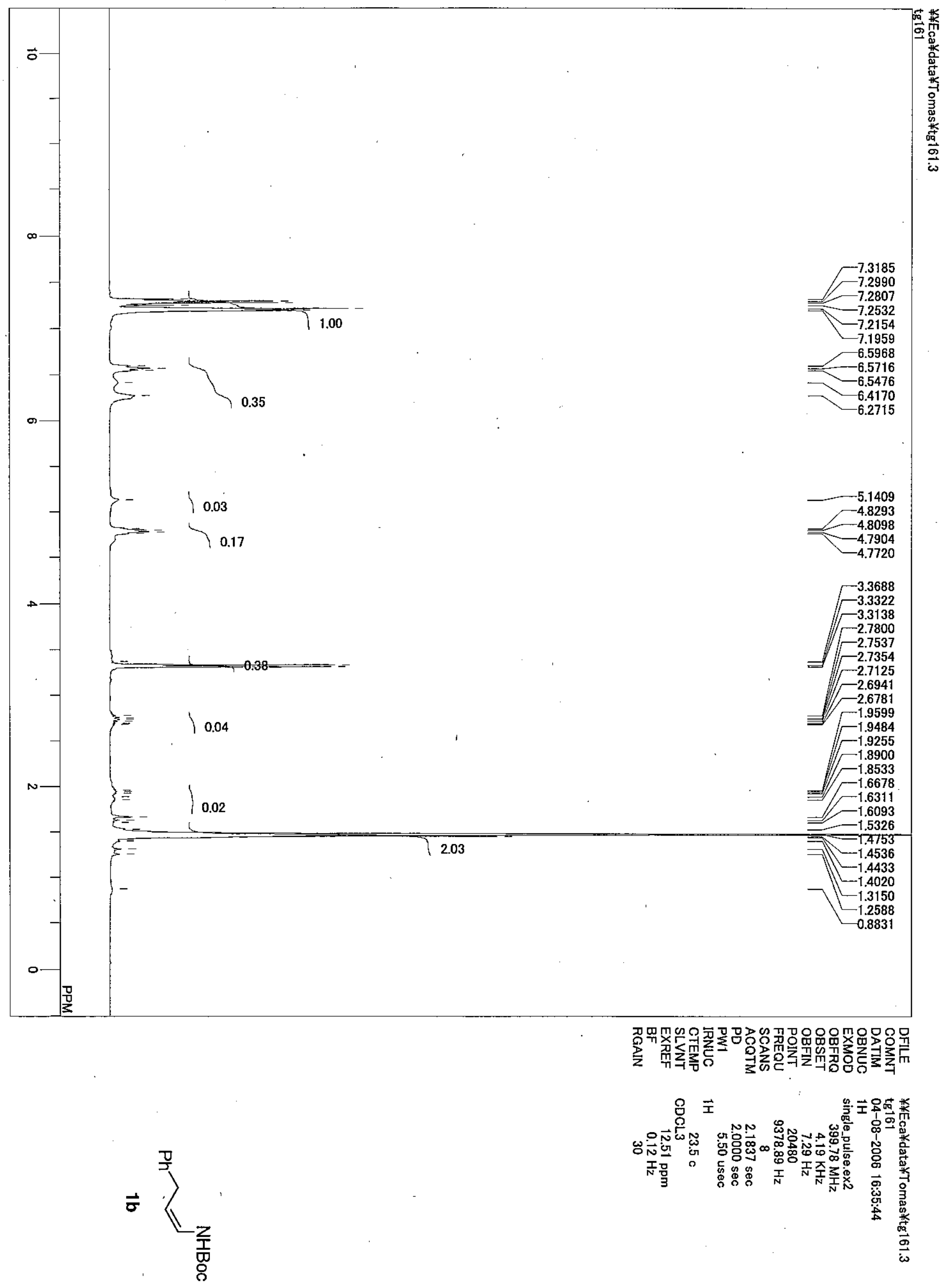




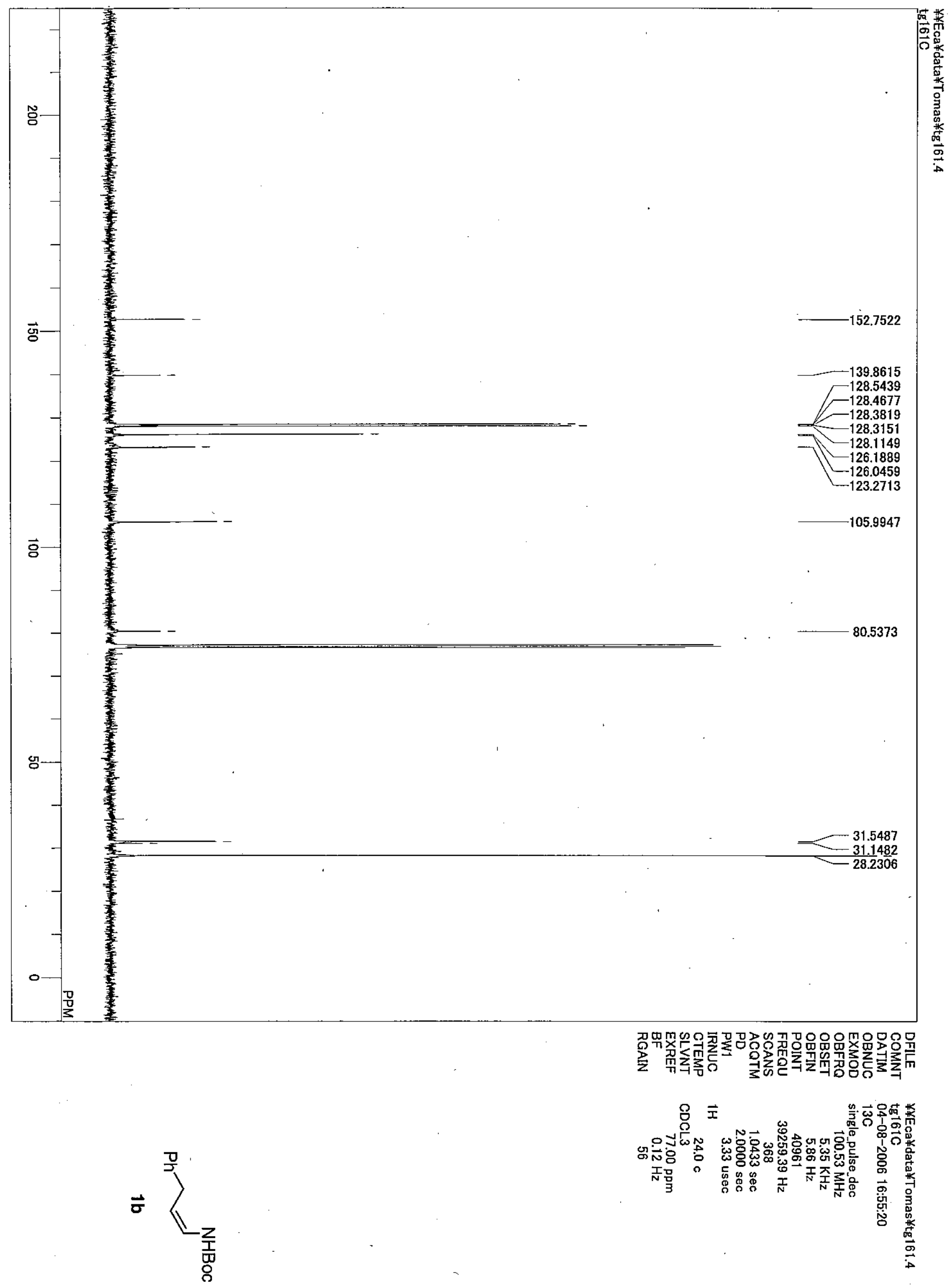




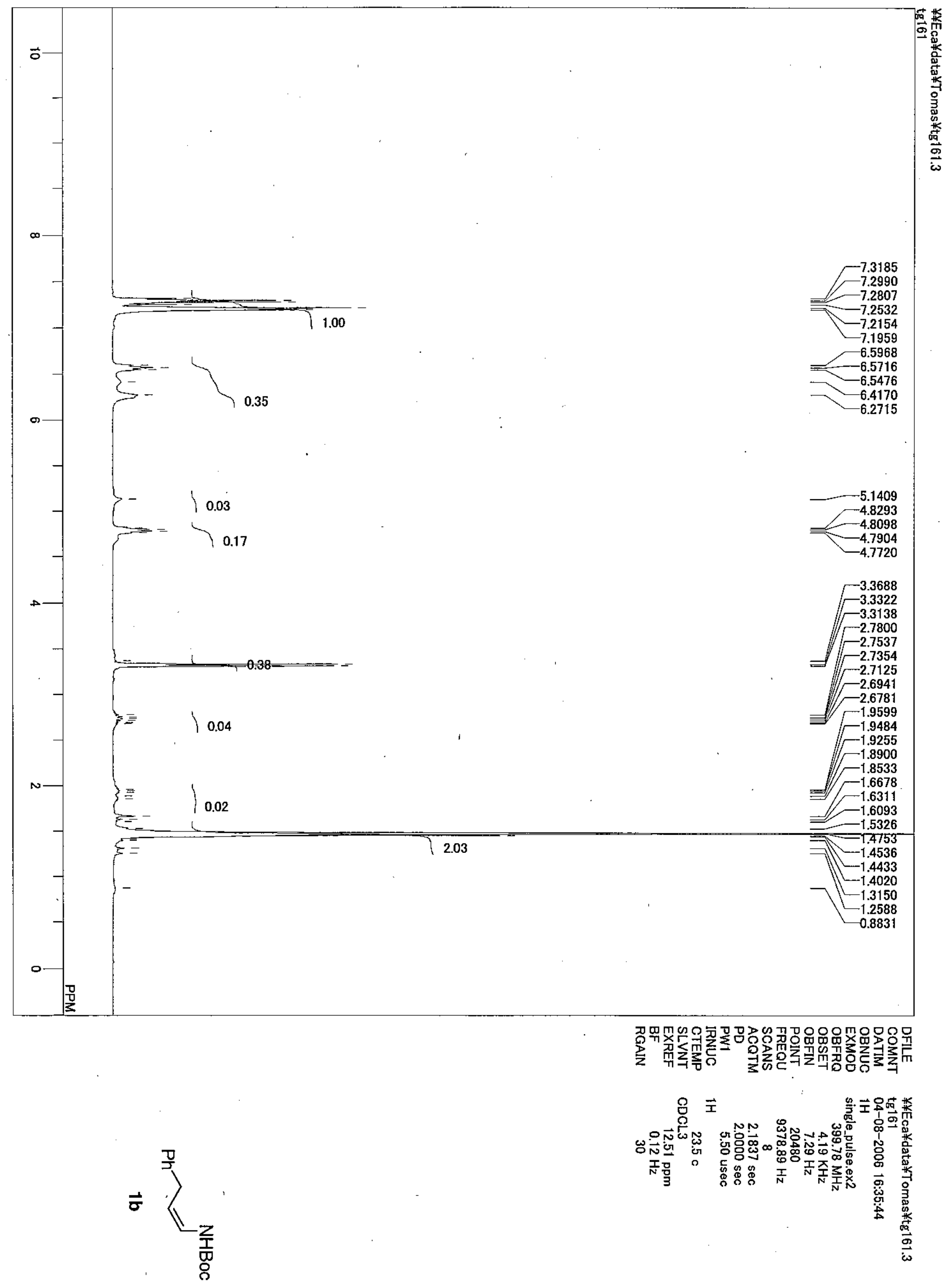




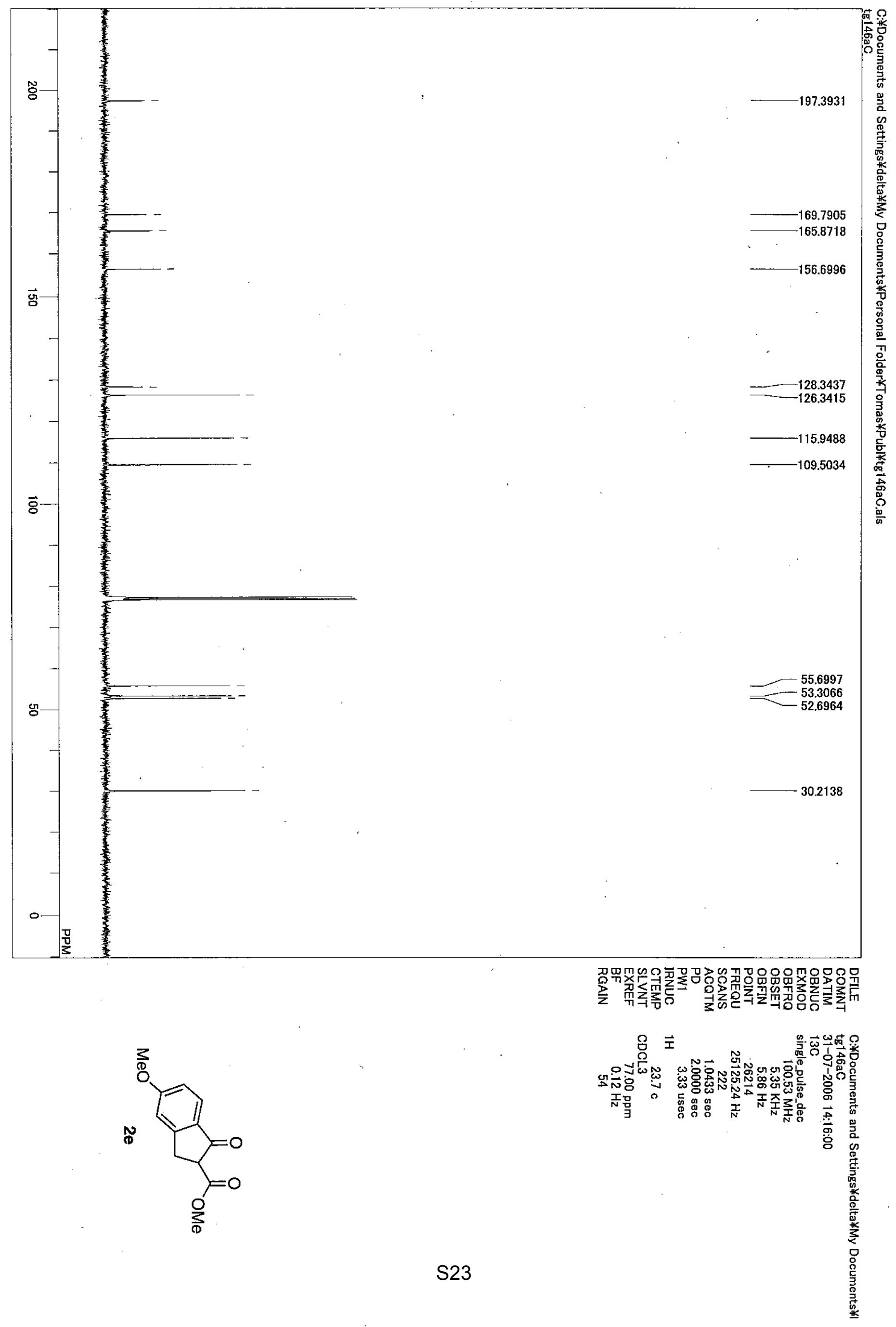




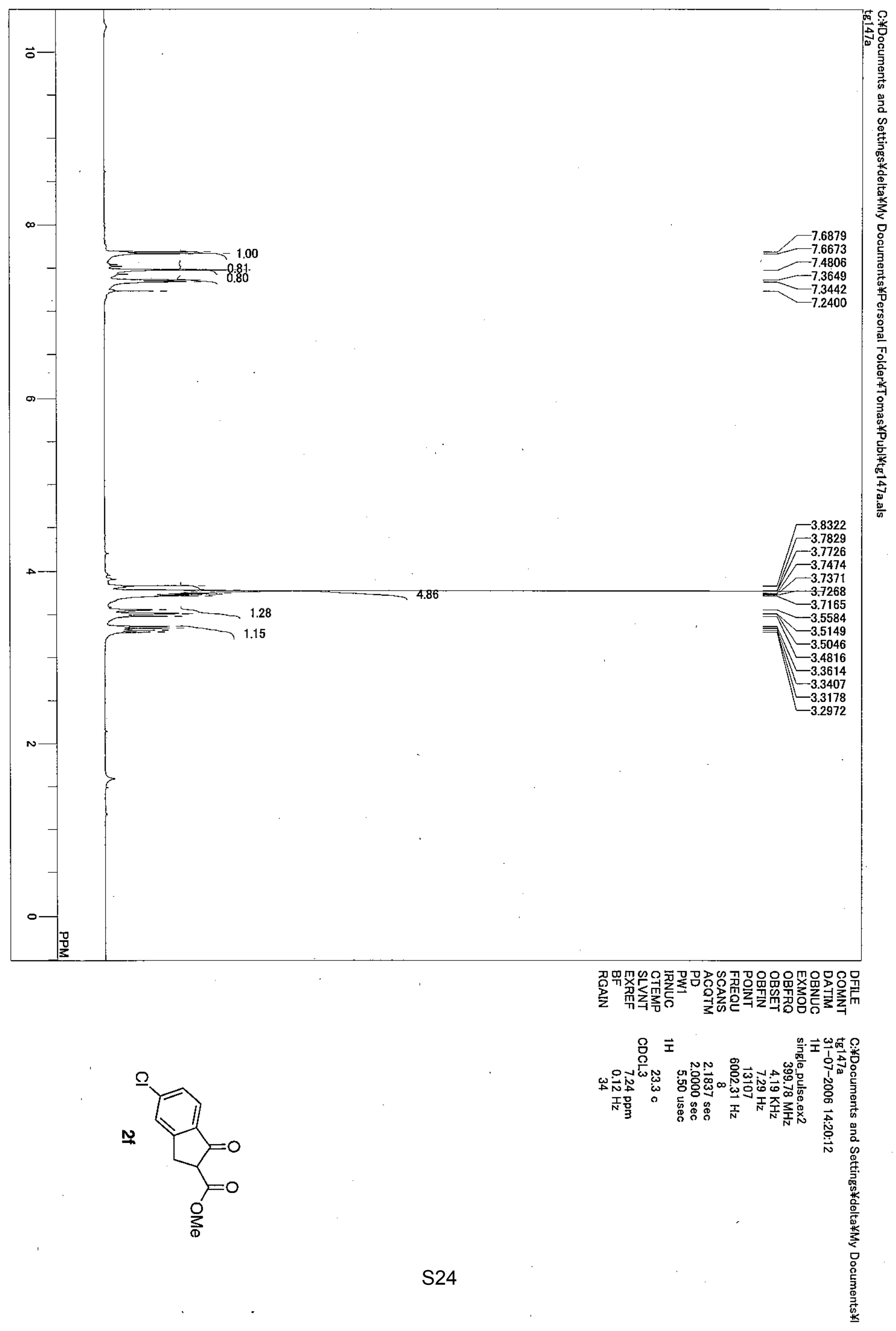




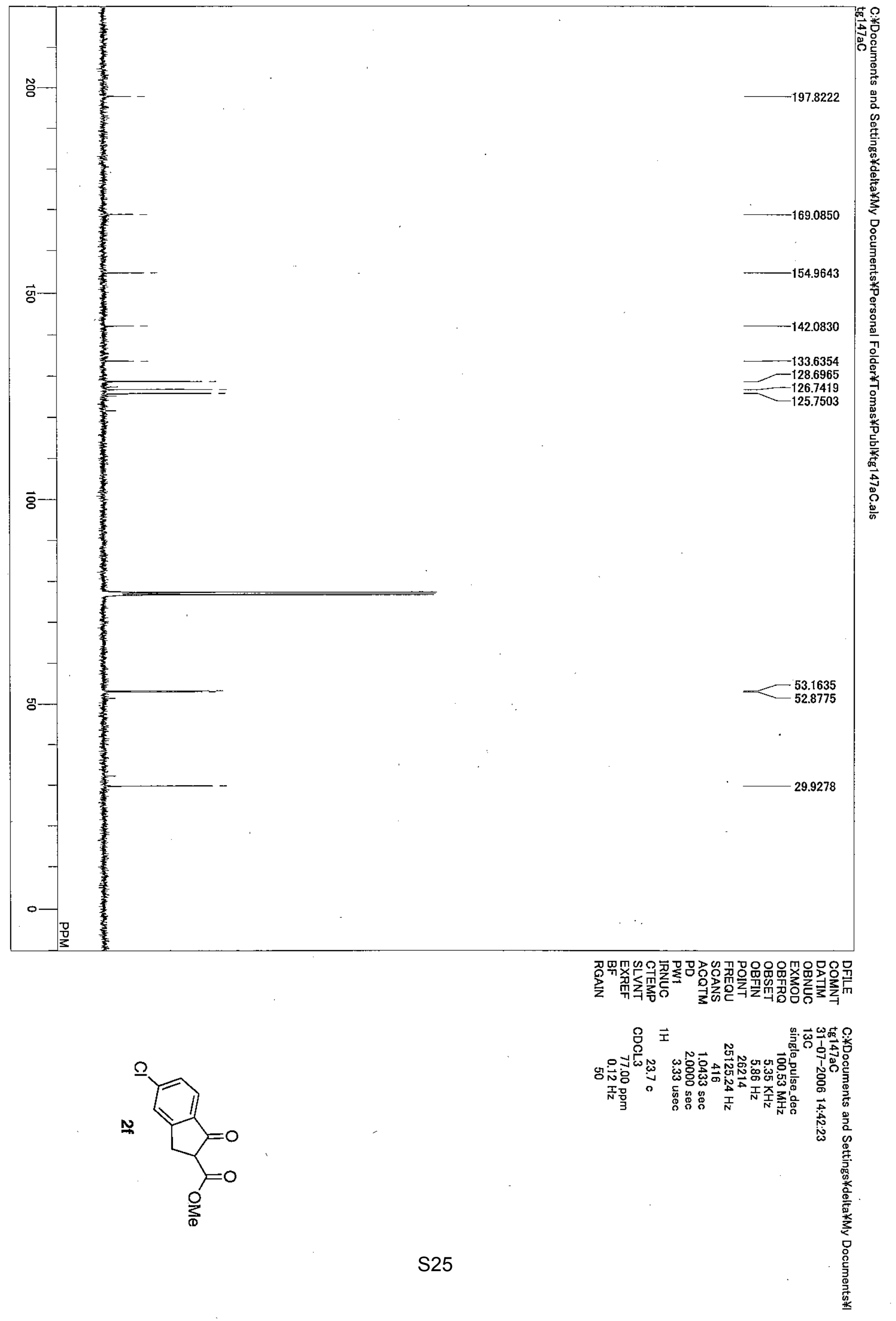




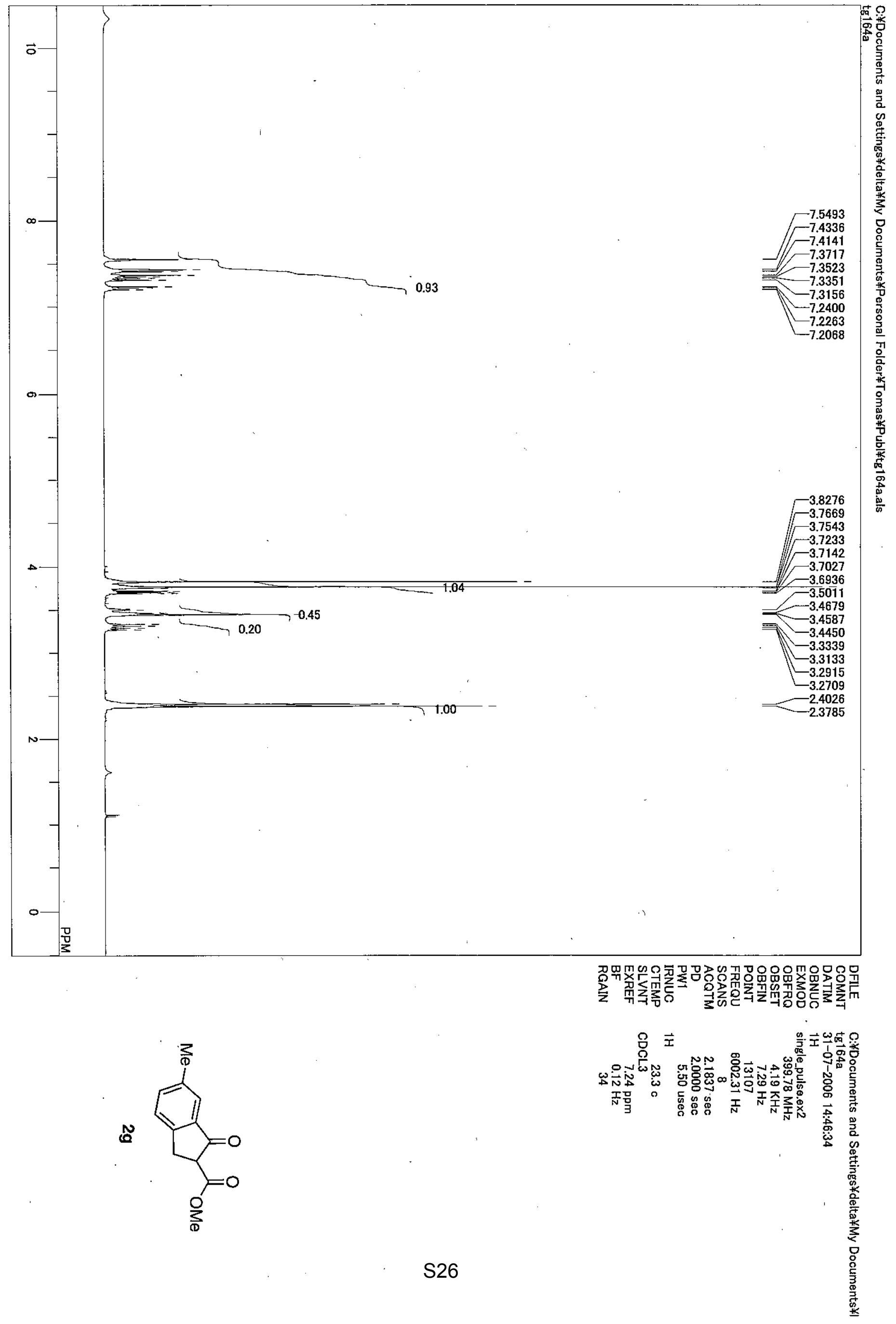




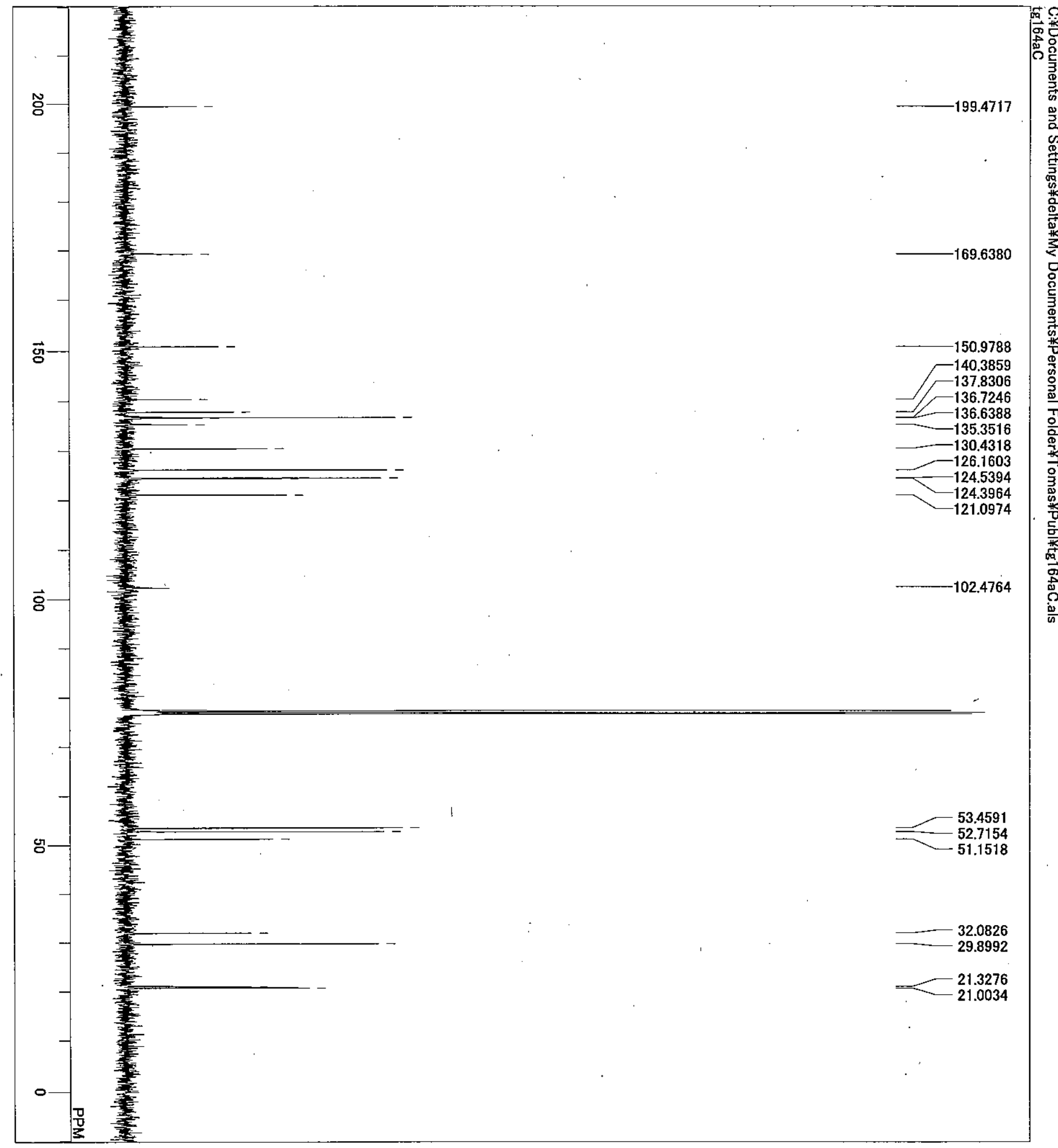

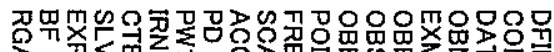

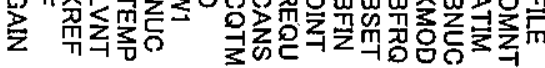<smiles>COC(=O)C1Cc2ccc(C)cc2C1=O</smiles>

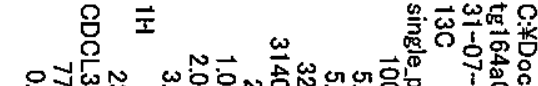

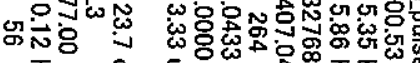

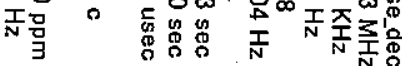




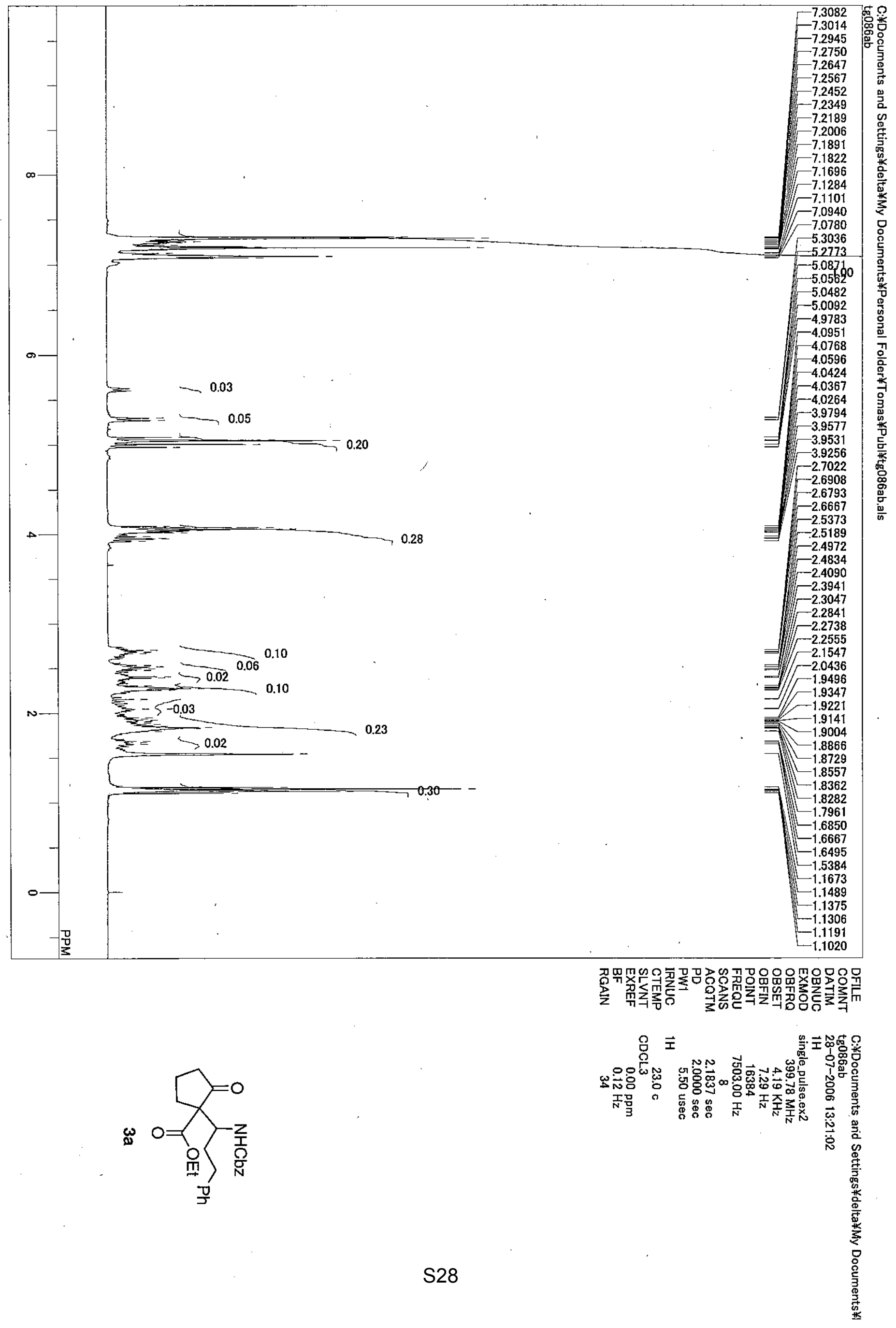




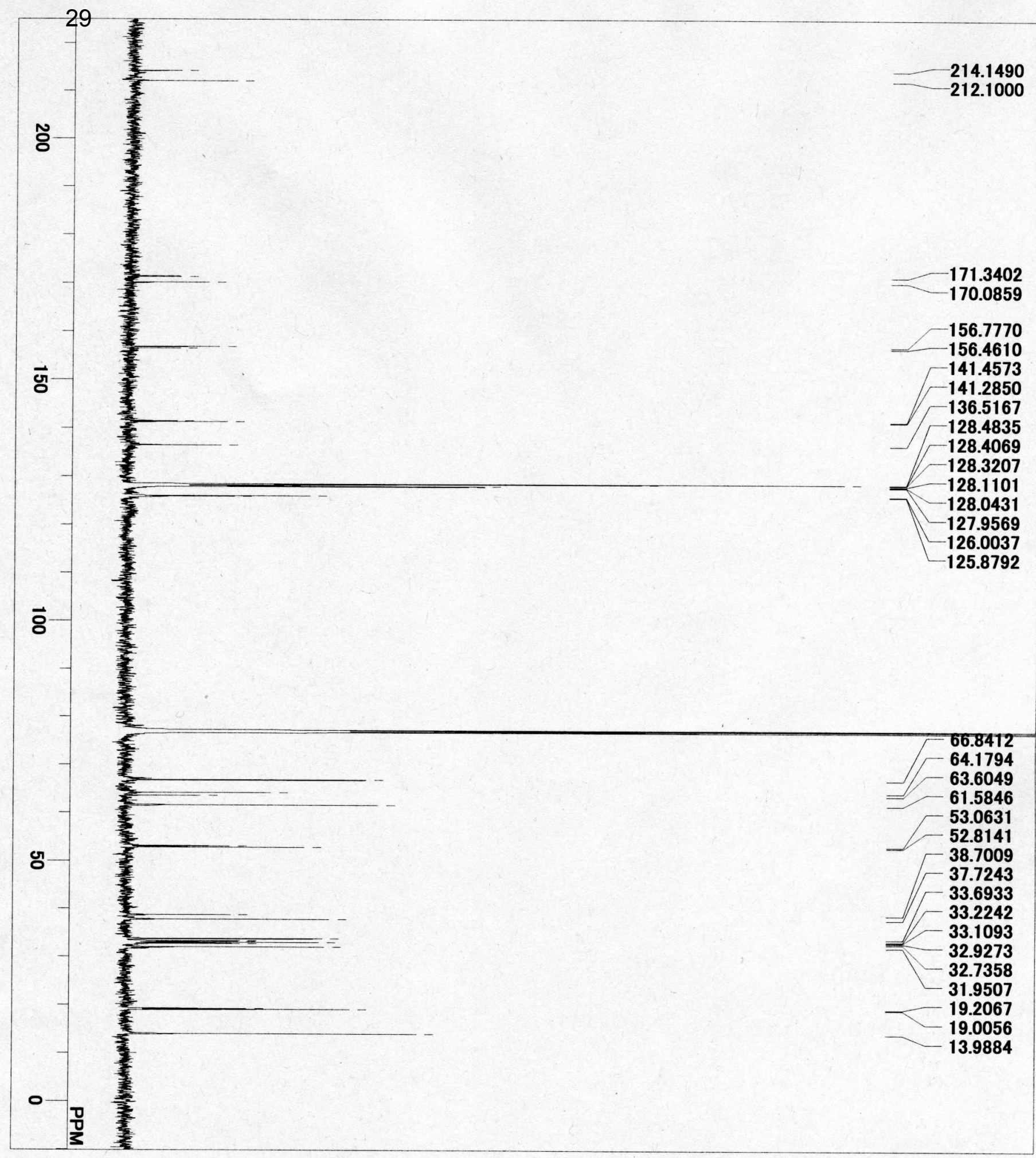

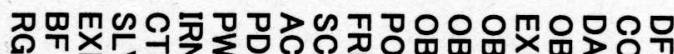

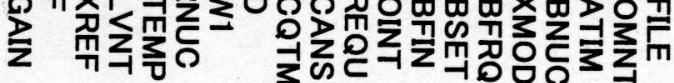<smiles>C=CC1(CC(=O)O)CCCC1=O</smiles> 


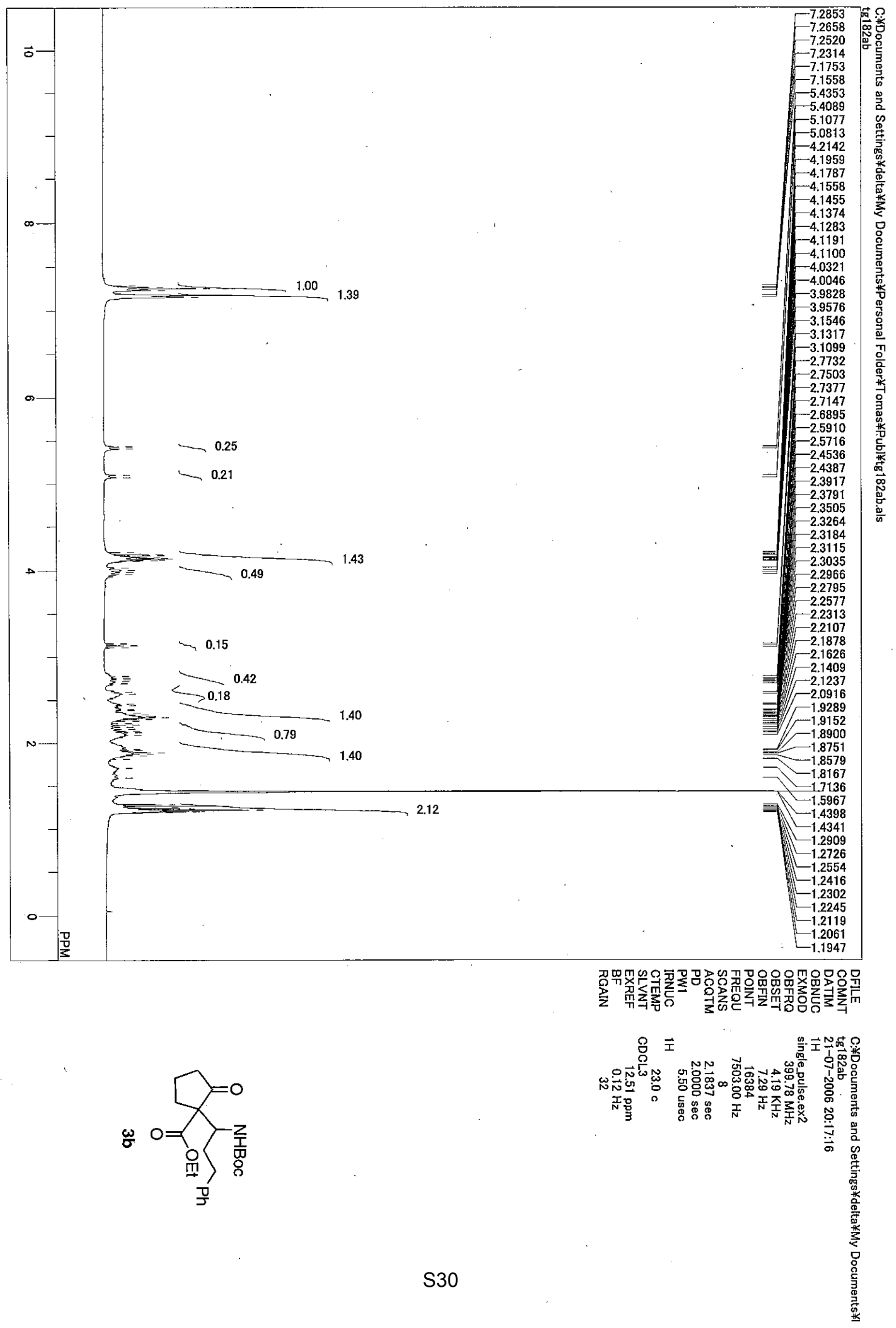




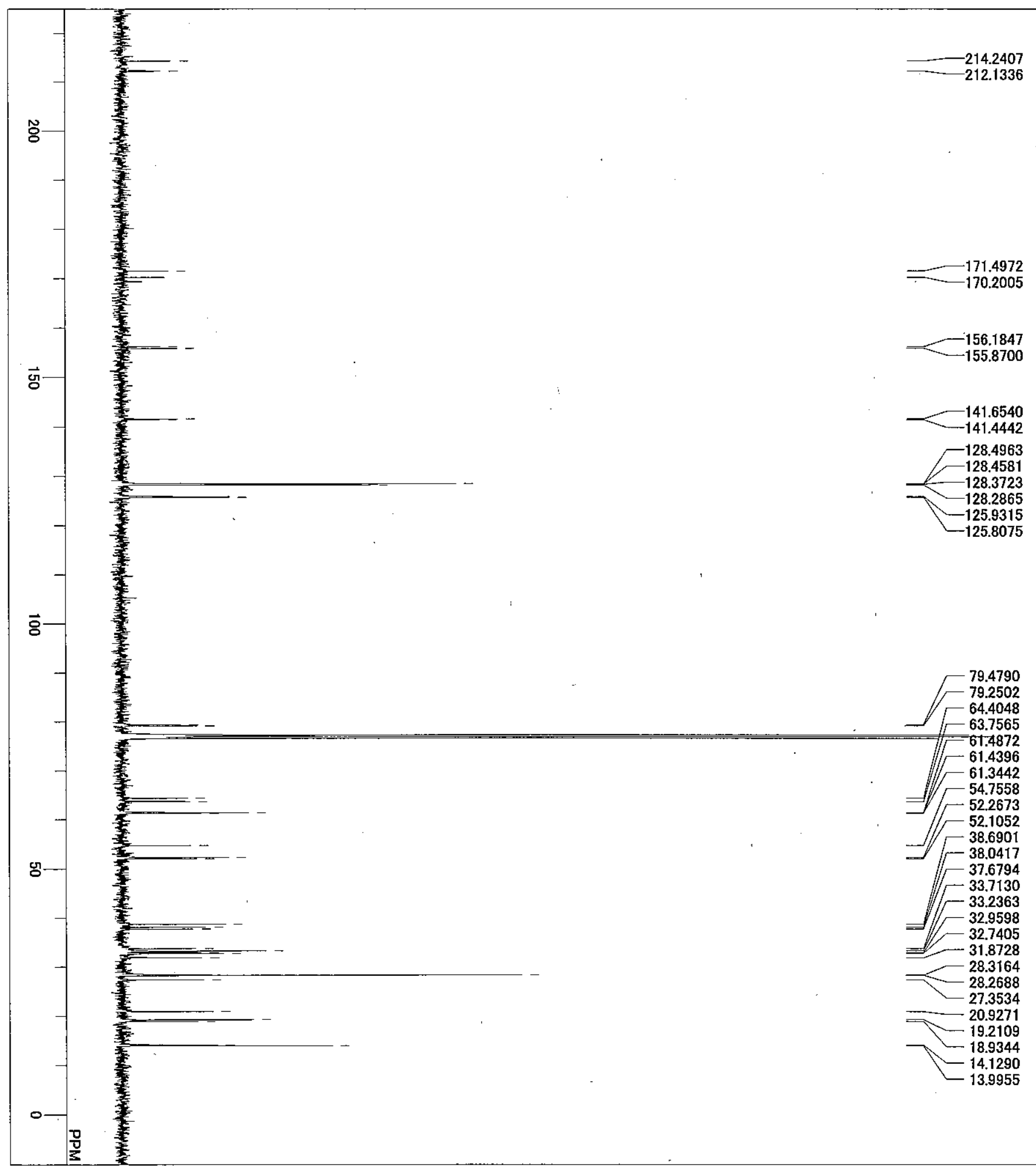

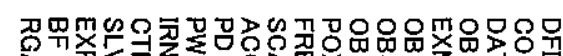

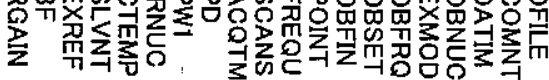<smiles>C#CCC(C(=O)O)C1(C(=O)O)CCCC1=O</smiles> 


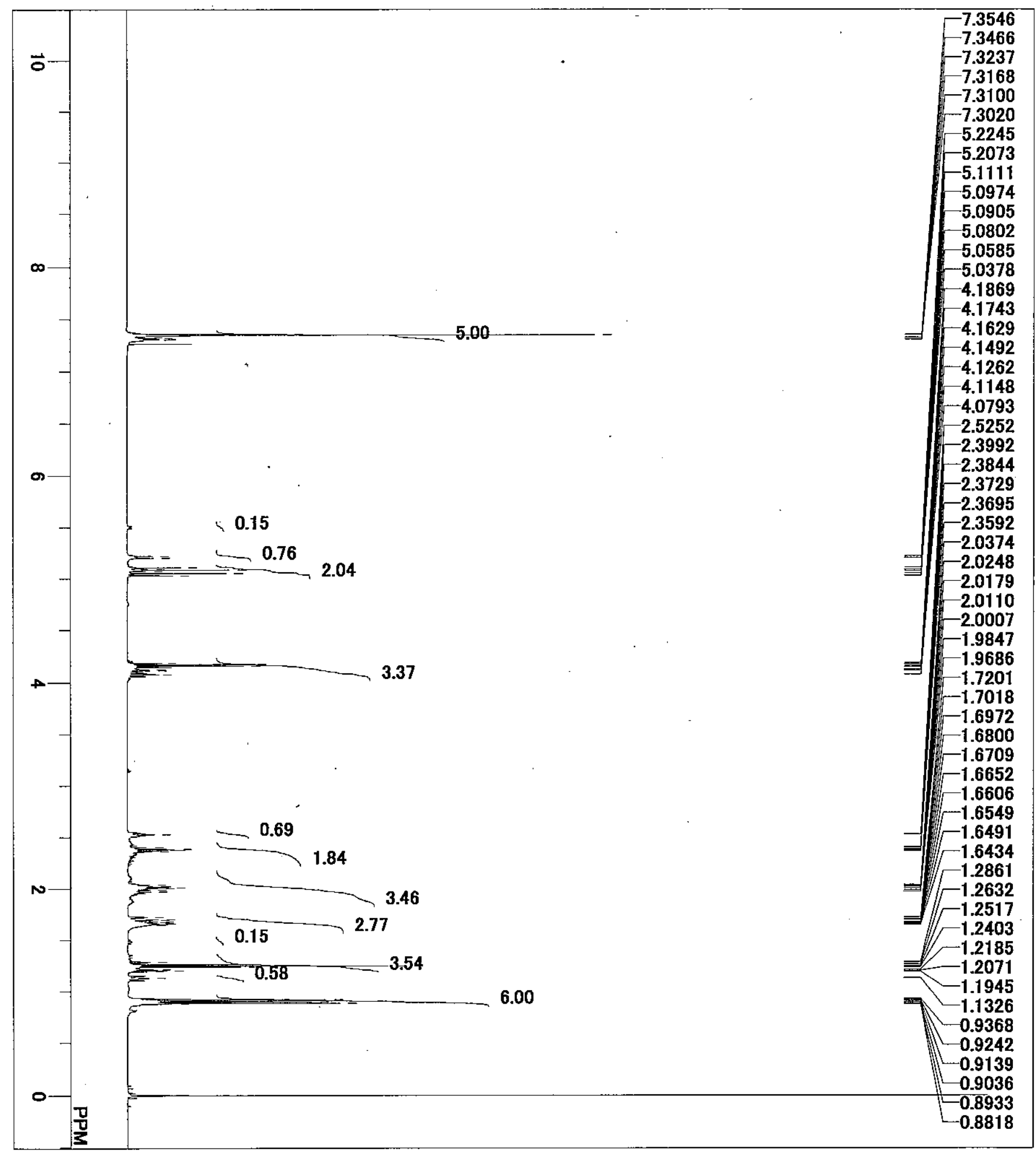

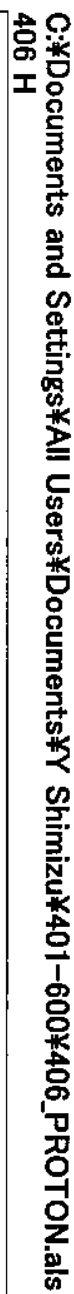

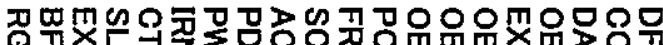

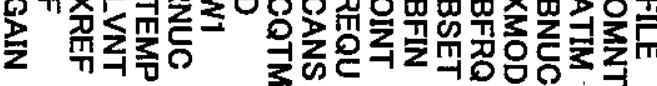
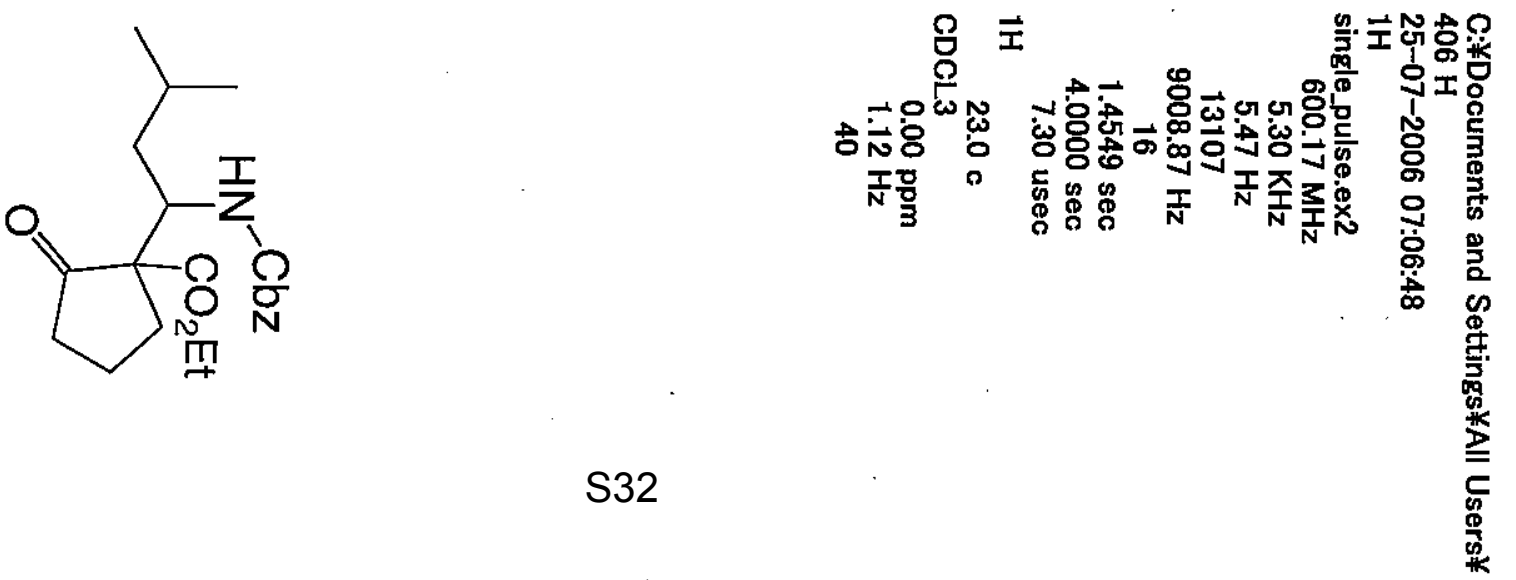

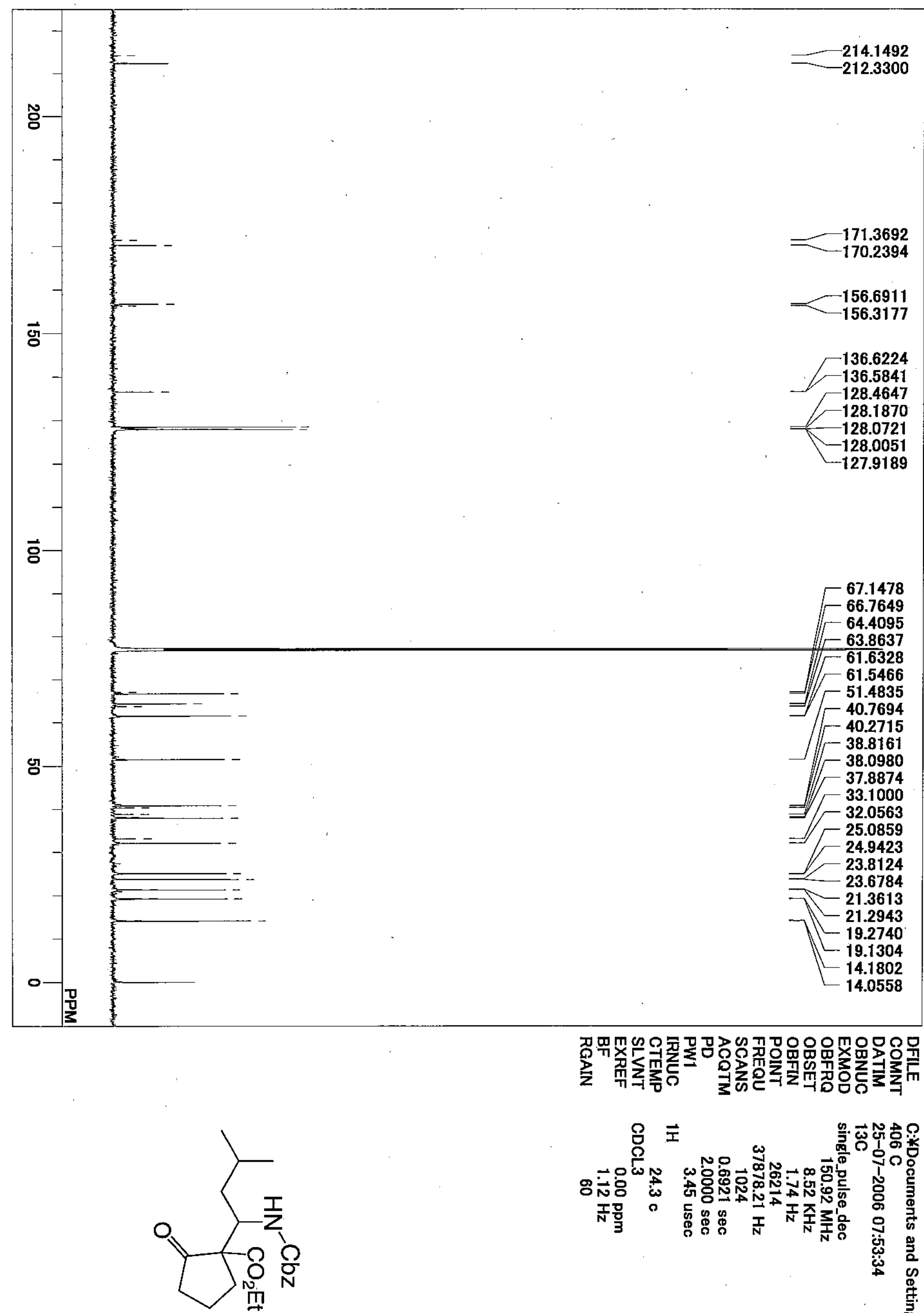


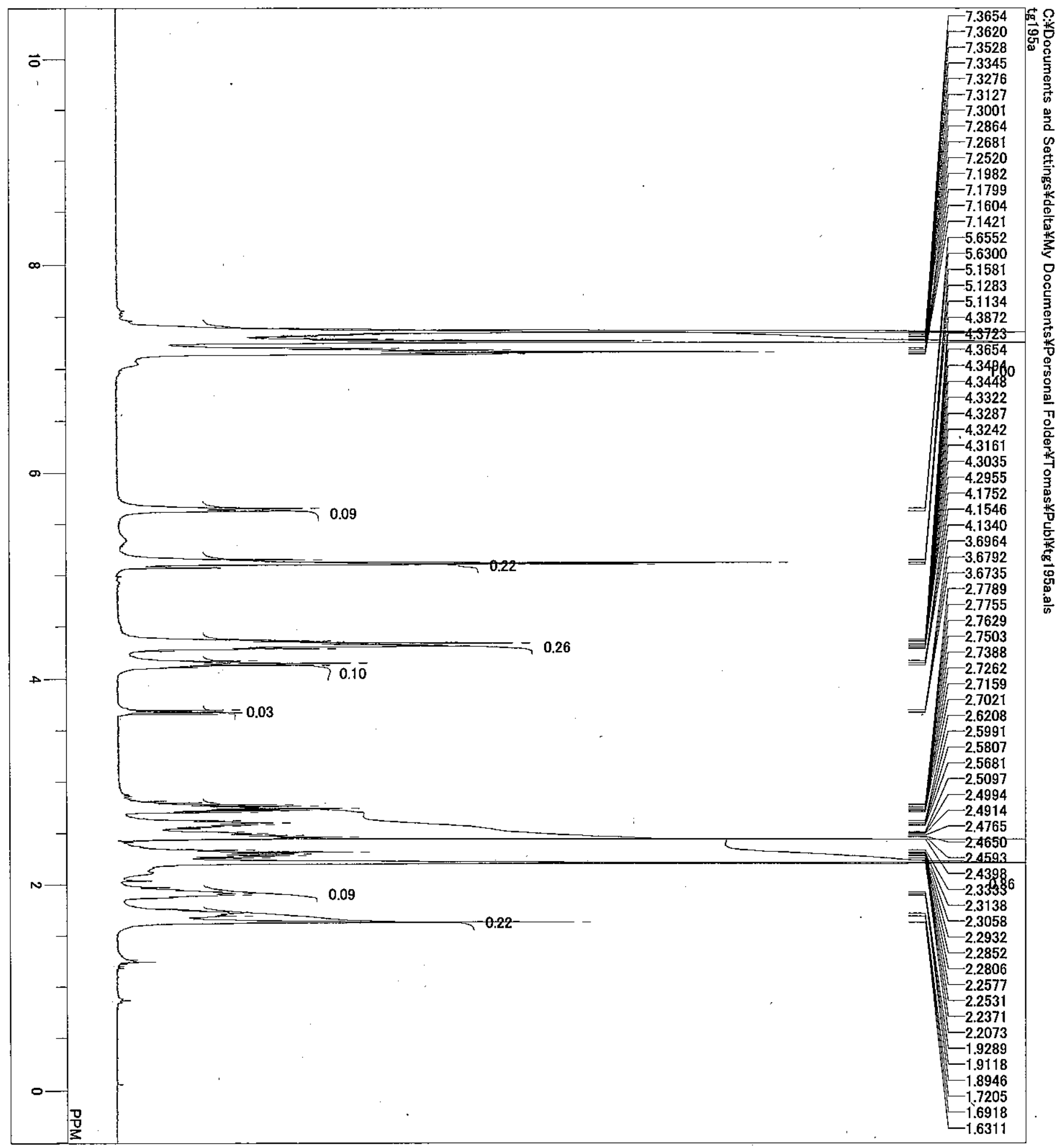

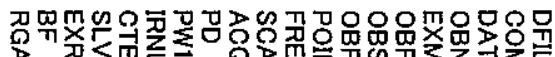

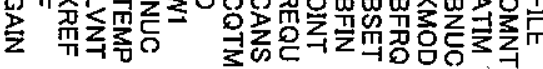<smiles>CCCC(CC)C1CCOC1=O</smiles> 


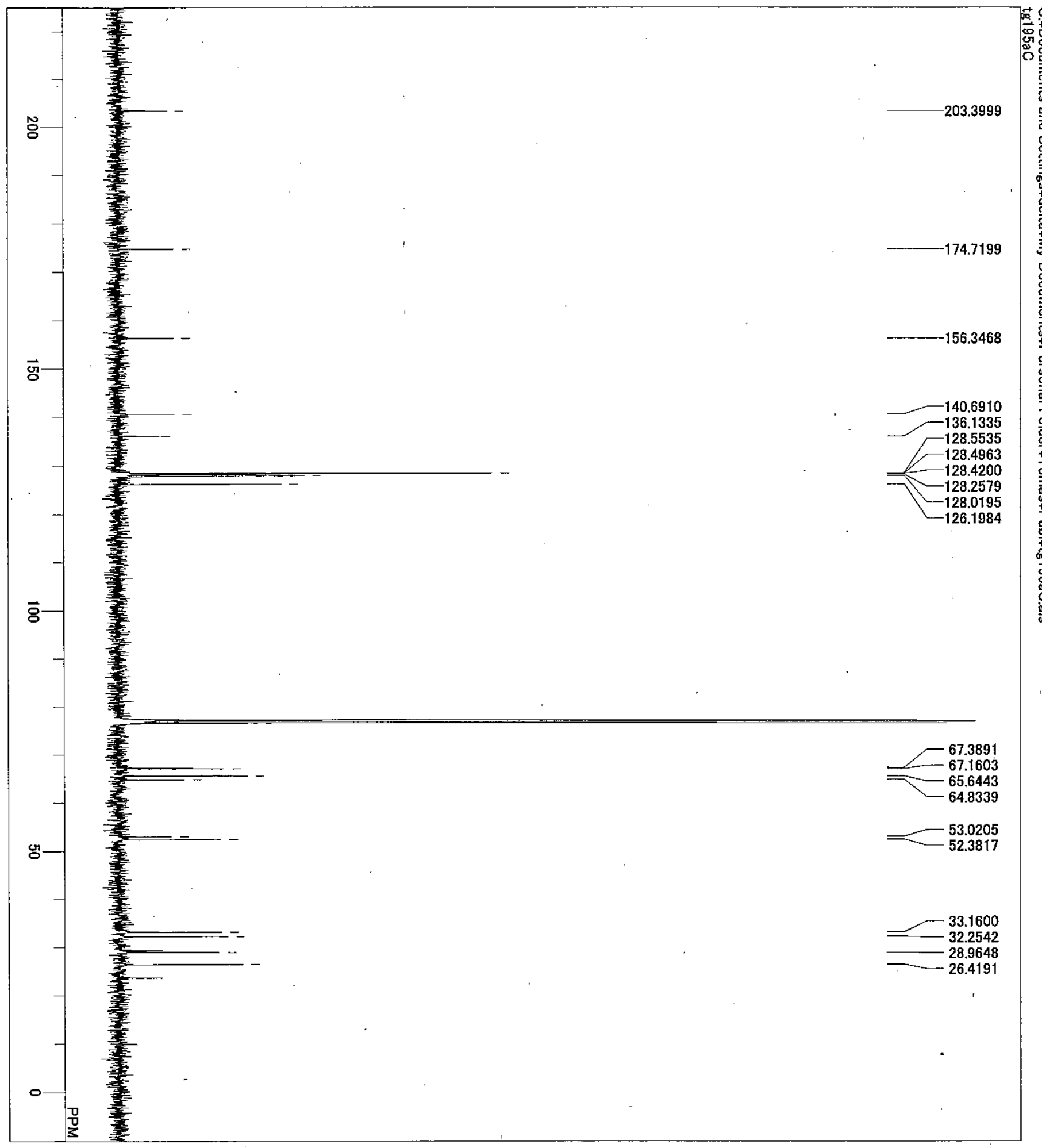

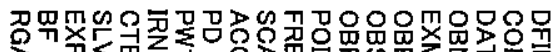

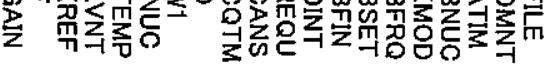<smiles>CC(=O)C1(C(C)C=O)CCOC1=O</smiles>

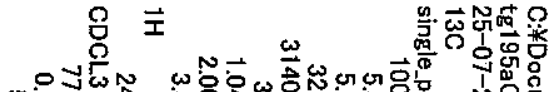

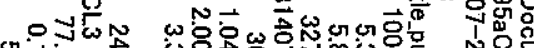
NO :

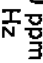




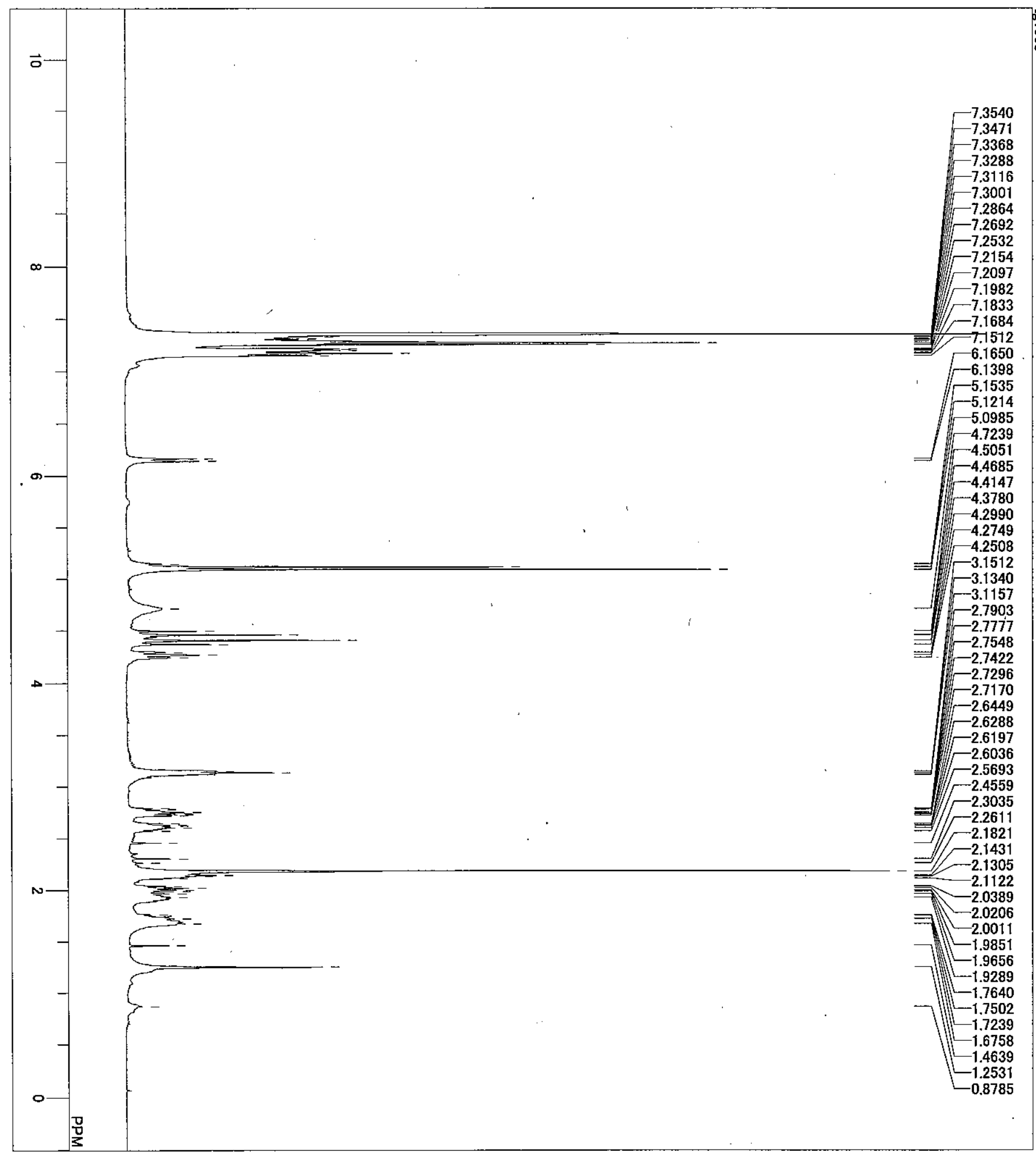

,

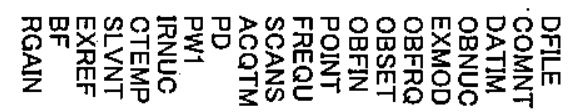<smiles>CC(=O)C1(C(C=O)C(=O)O)CC[C@@H](O)C1=O</smiles> 


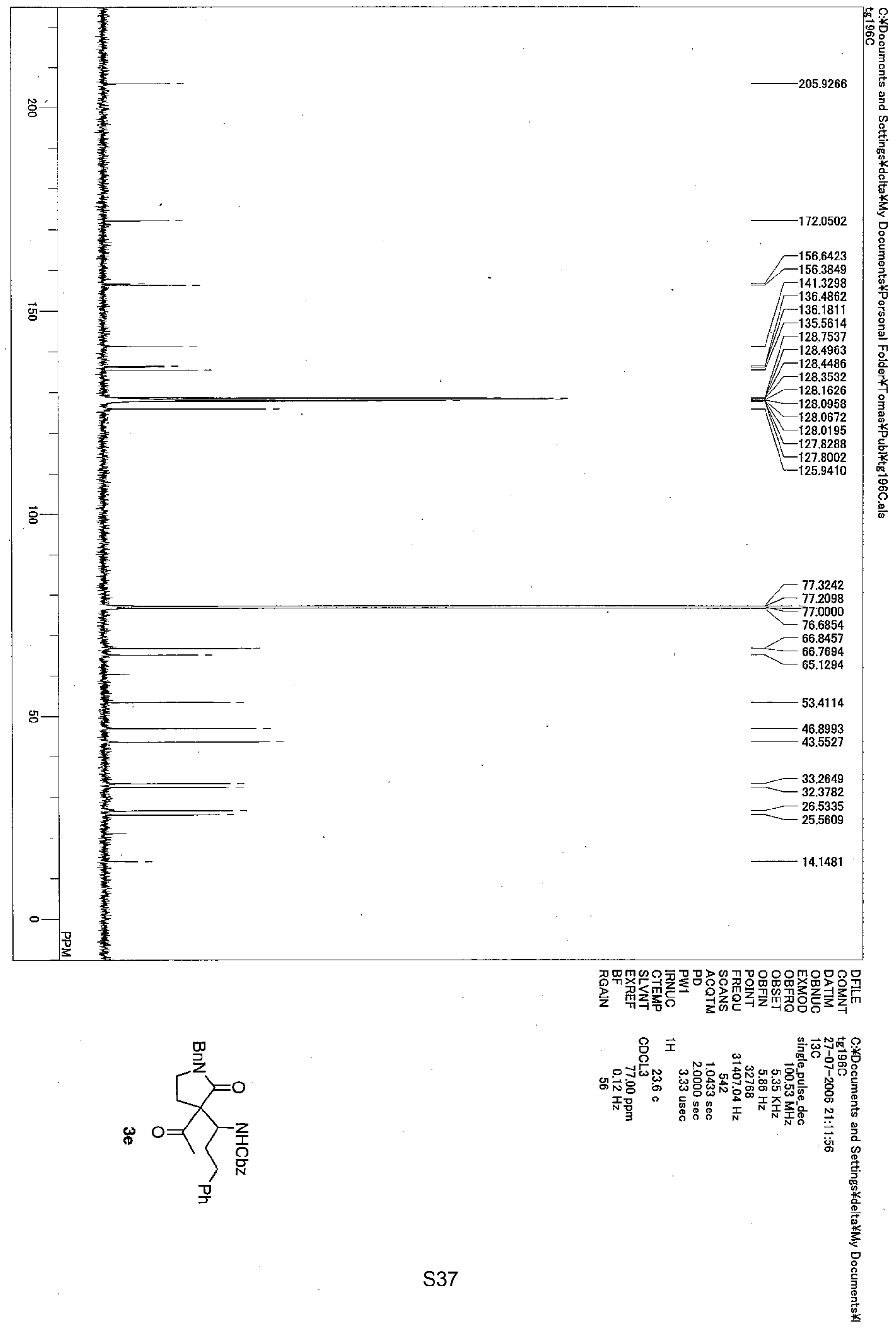




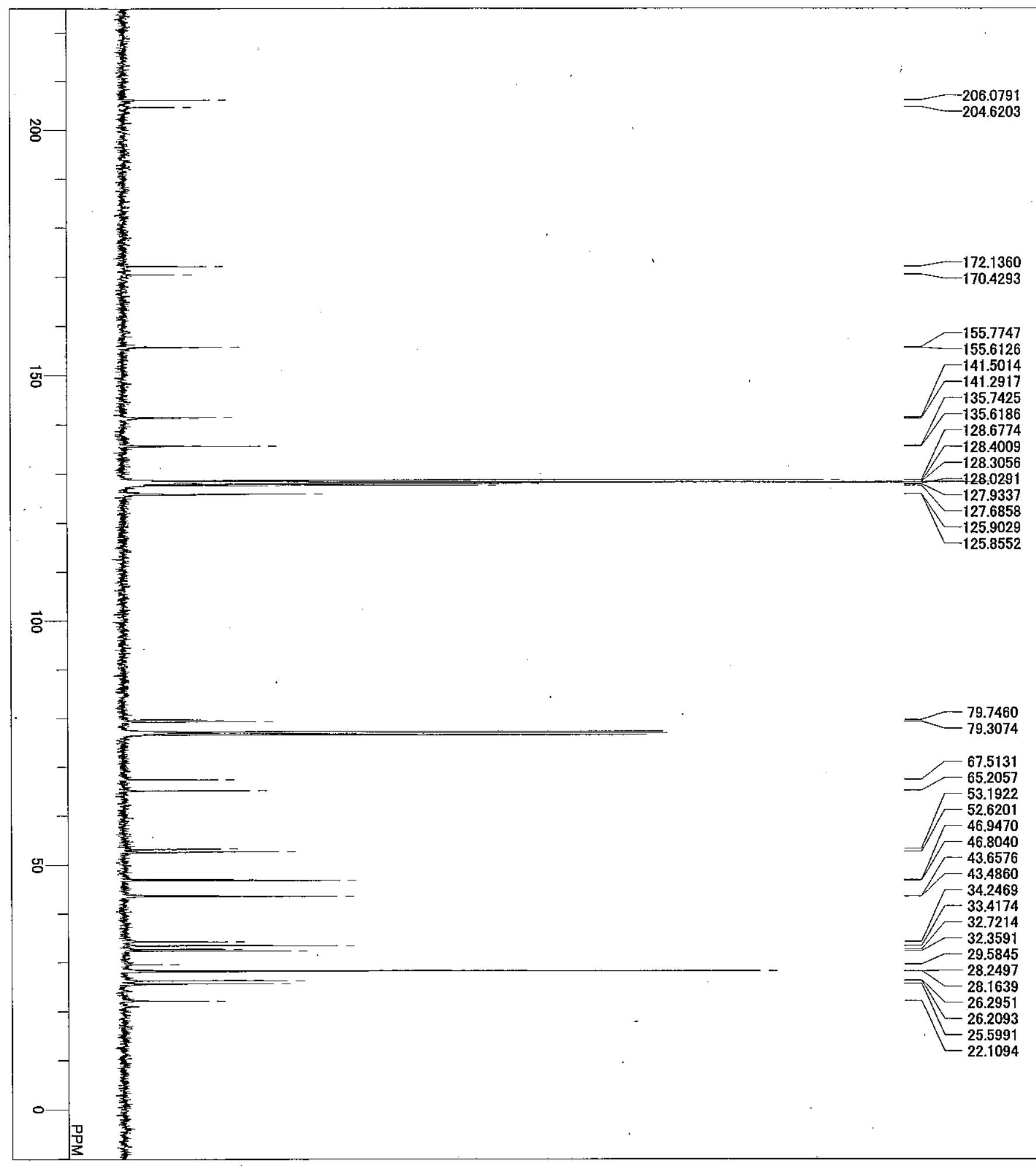

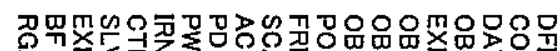

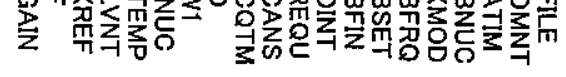<smiles>CCCC(C(C)=O)C1(C(C)=O)CC[C@@H](C)C1=O</smiles>

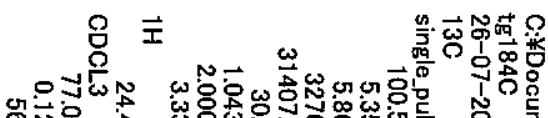

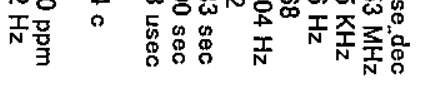




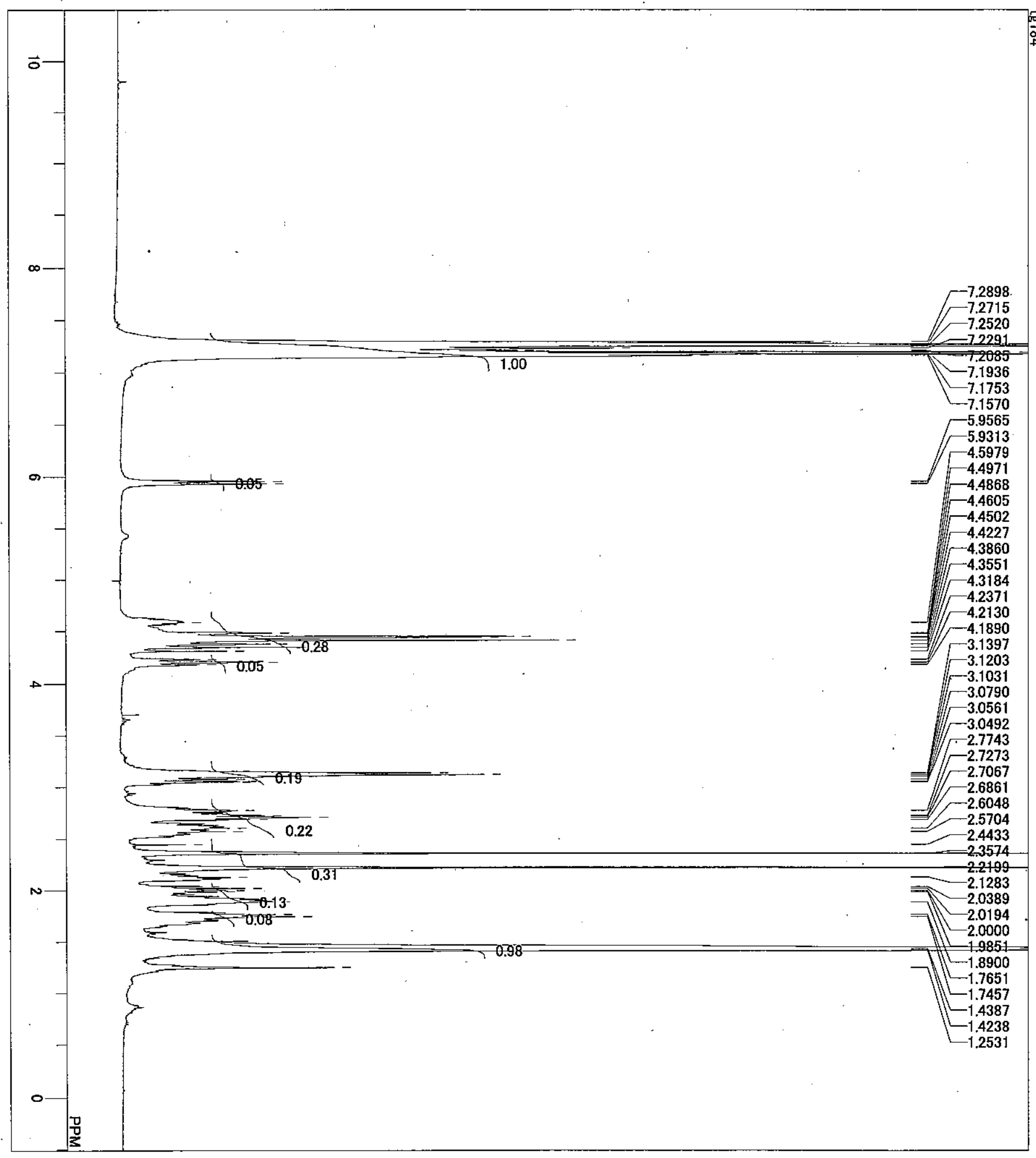

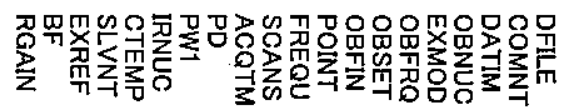<smiles>CCCC(C(=O)O)C1(C(C)=O)CC[C@@H](O)C1=O</smiles>

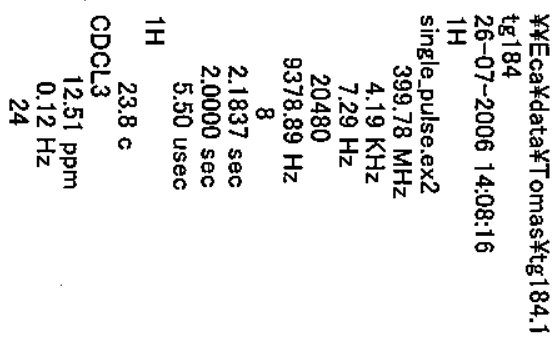




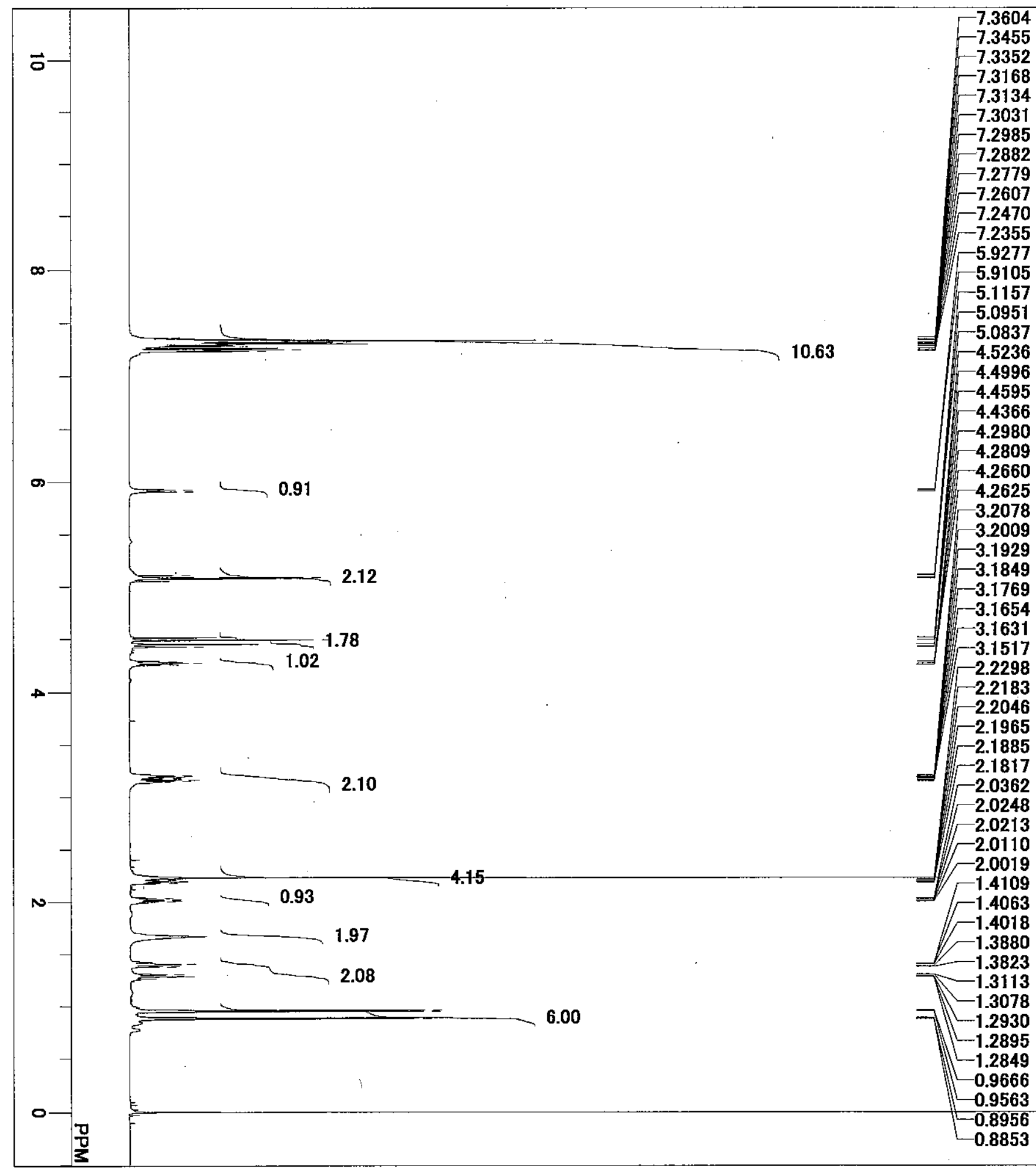

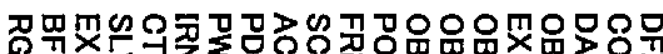

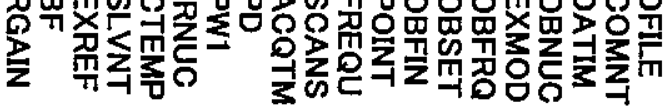<smiles>C[C](C)C(CC(C)C)C1(C(=O)[O-])CC[C@H](O)C1=O</smiles>

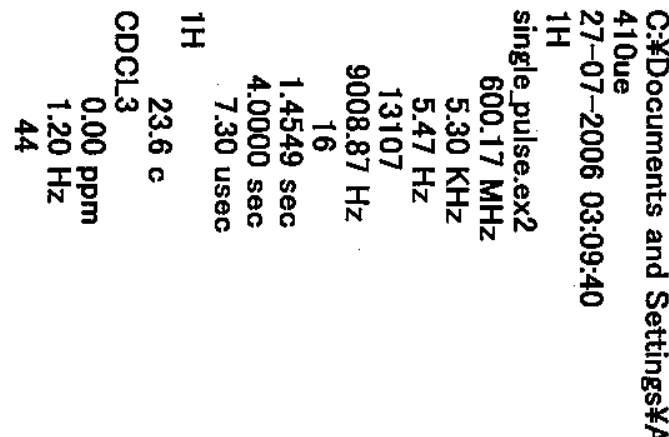




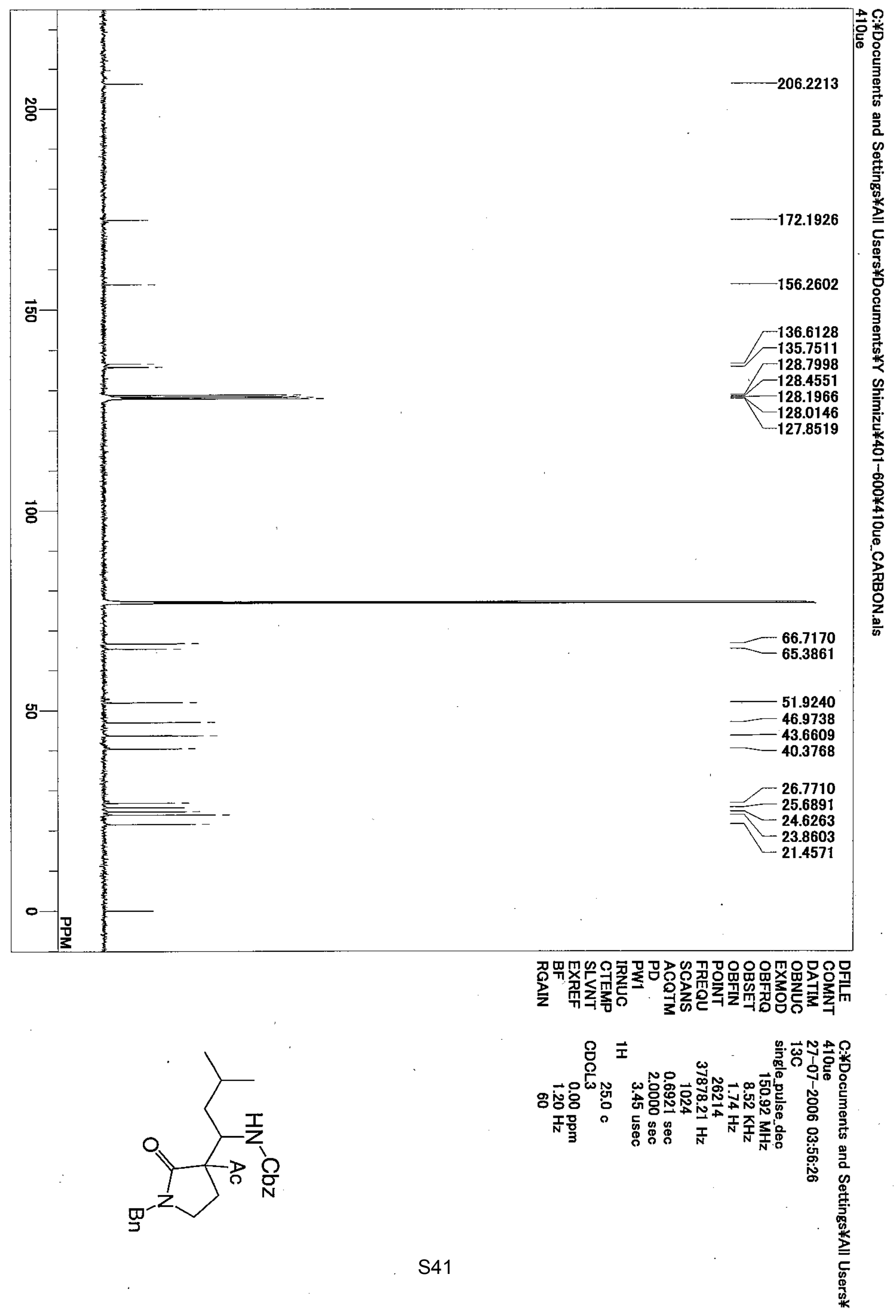



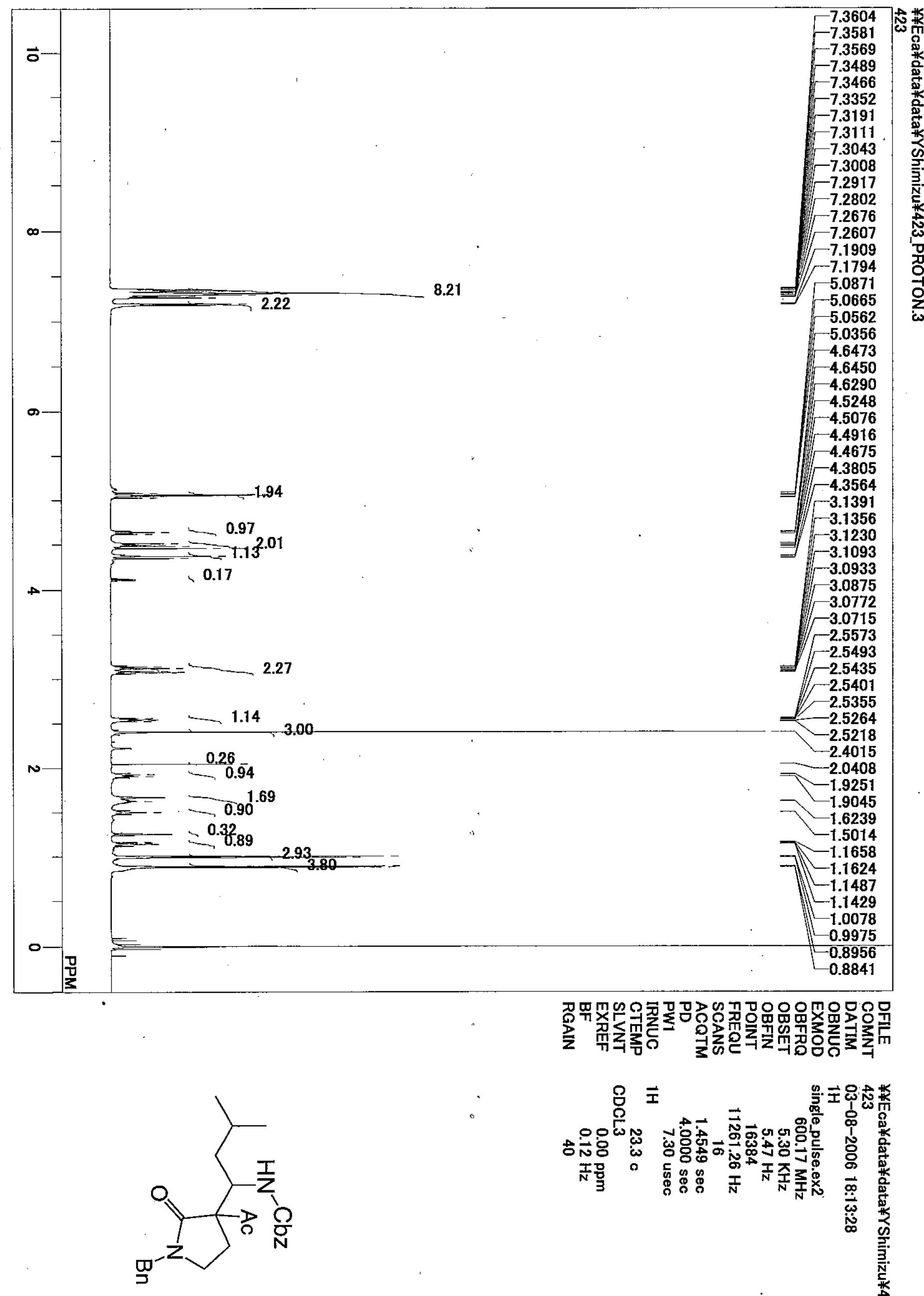


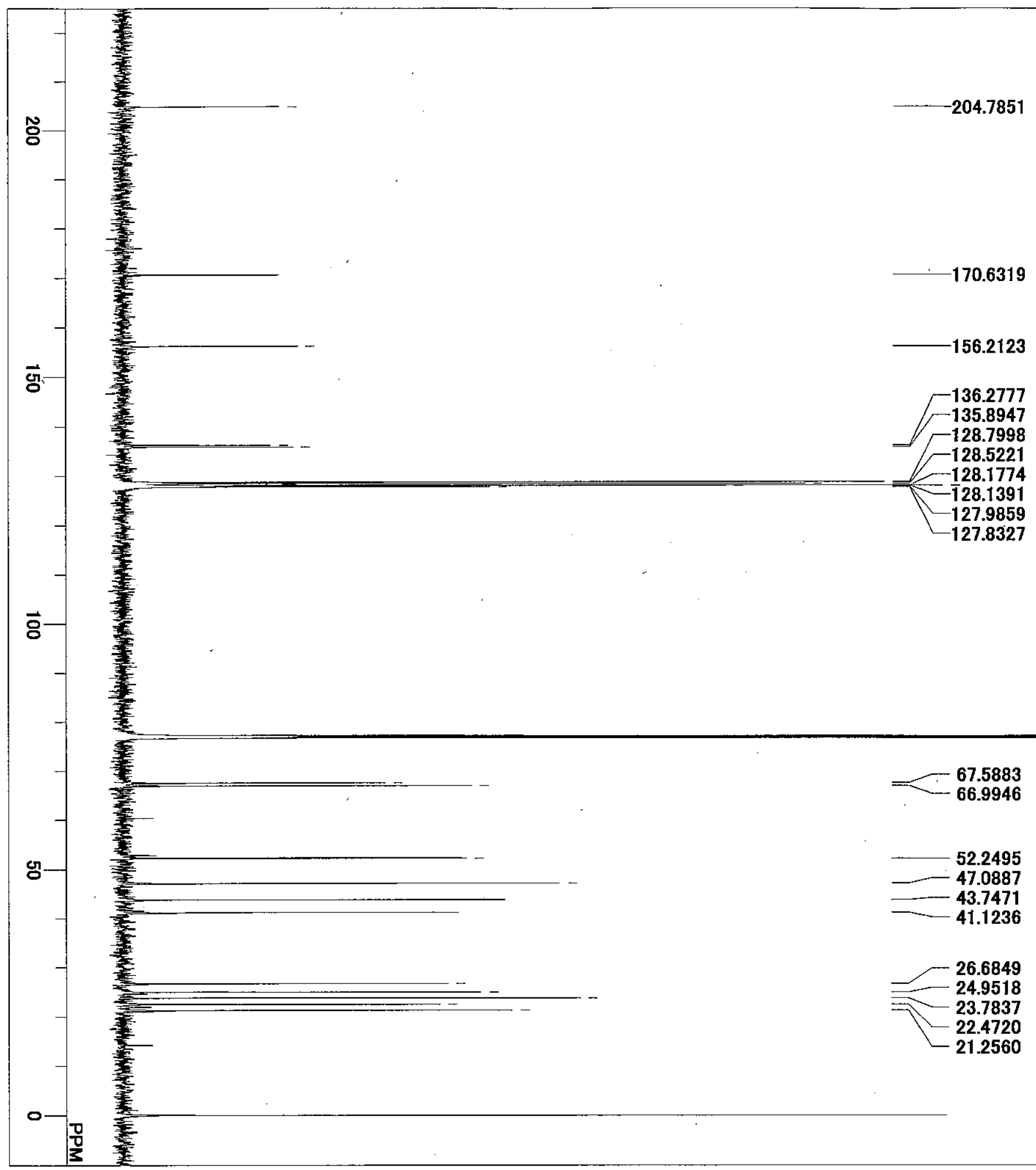

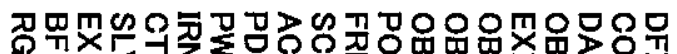

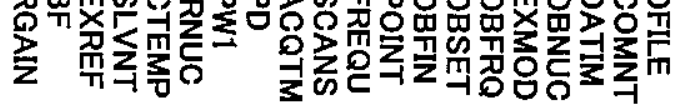

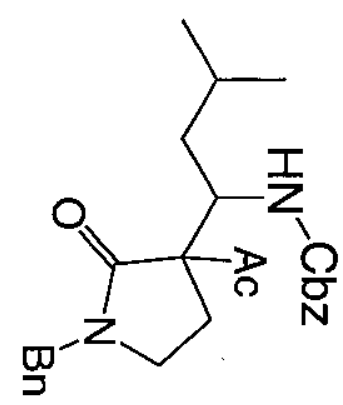




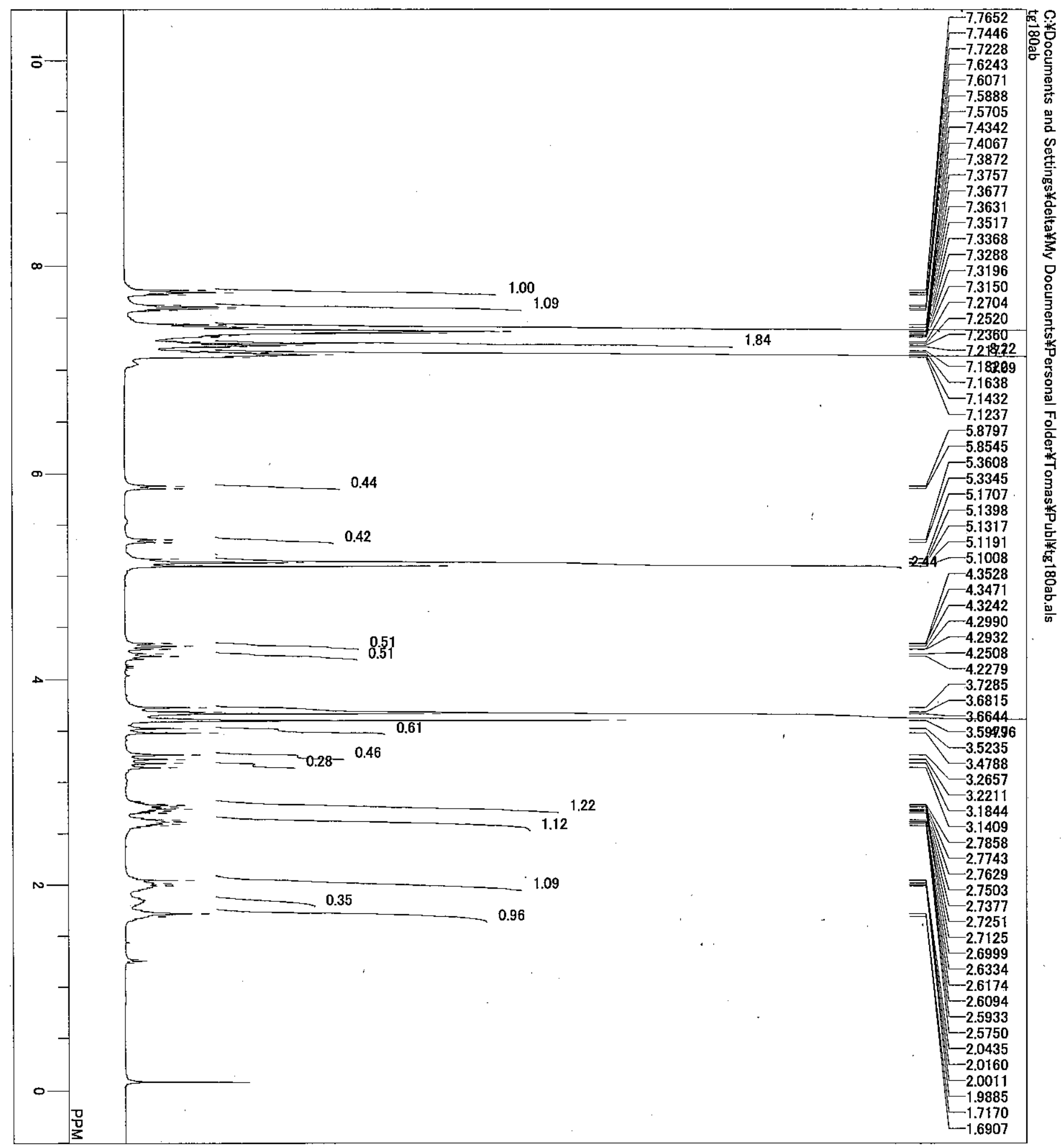

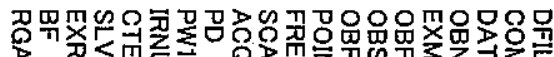

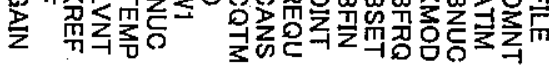

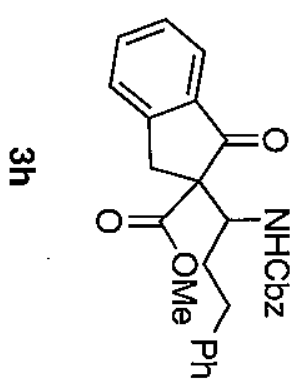




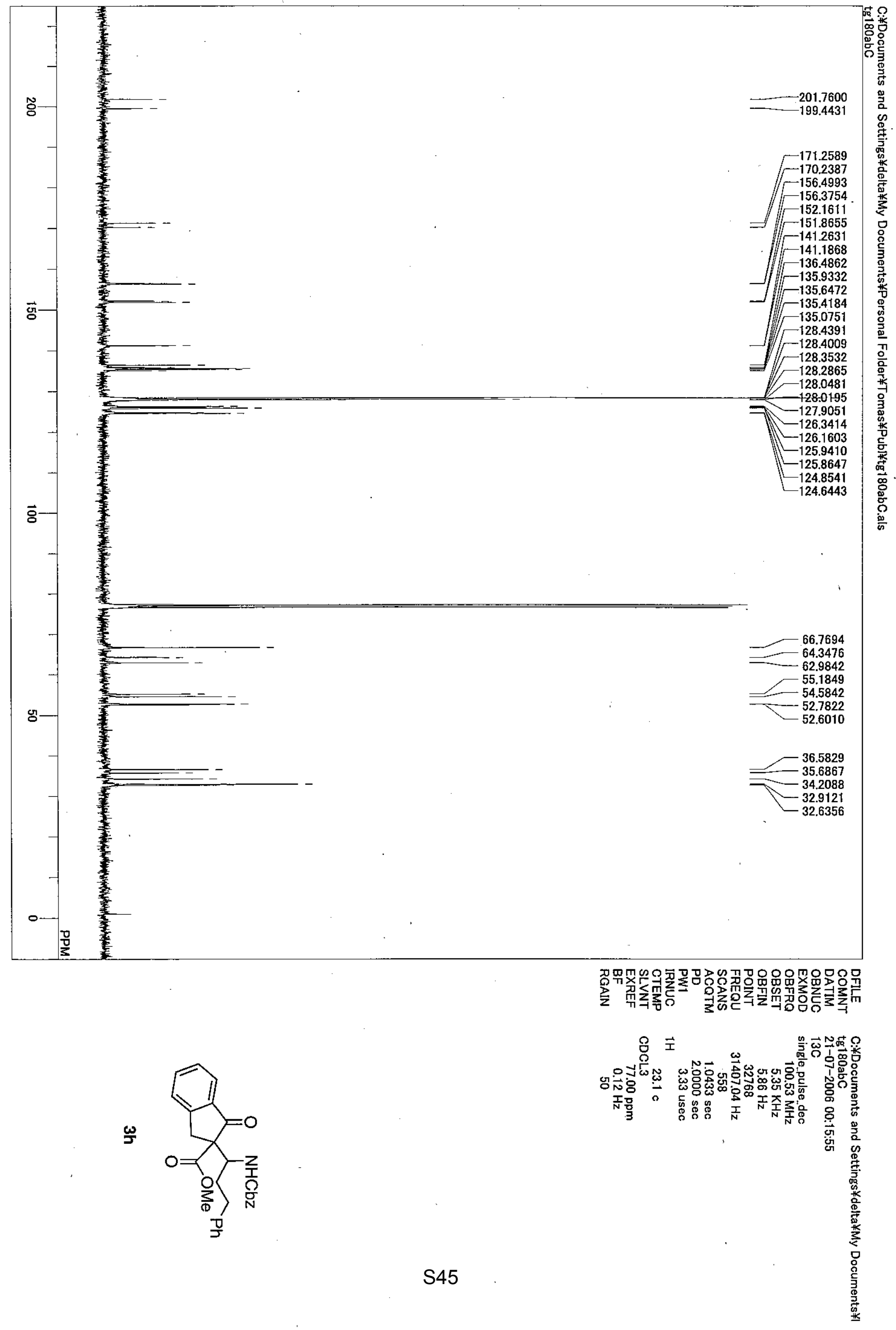




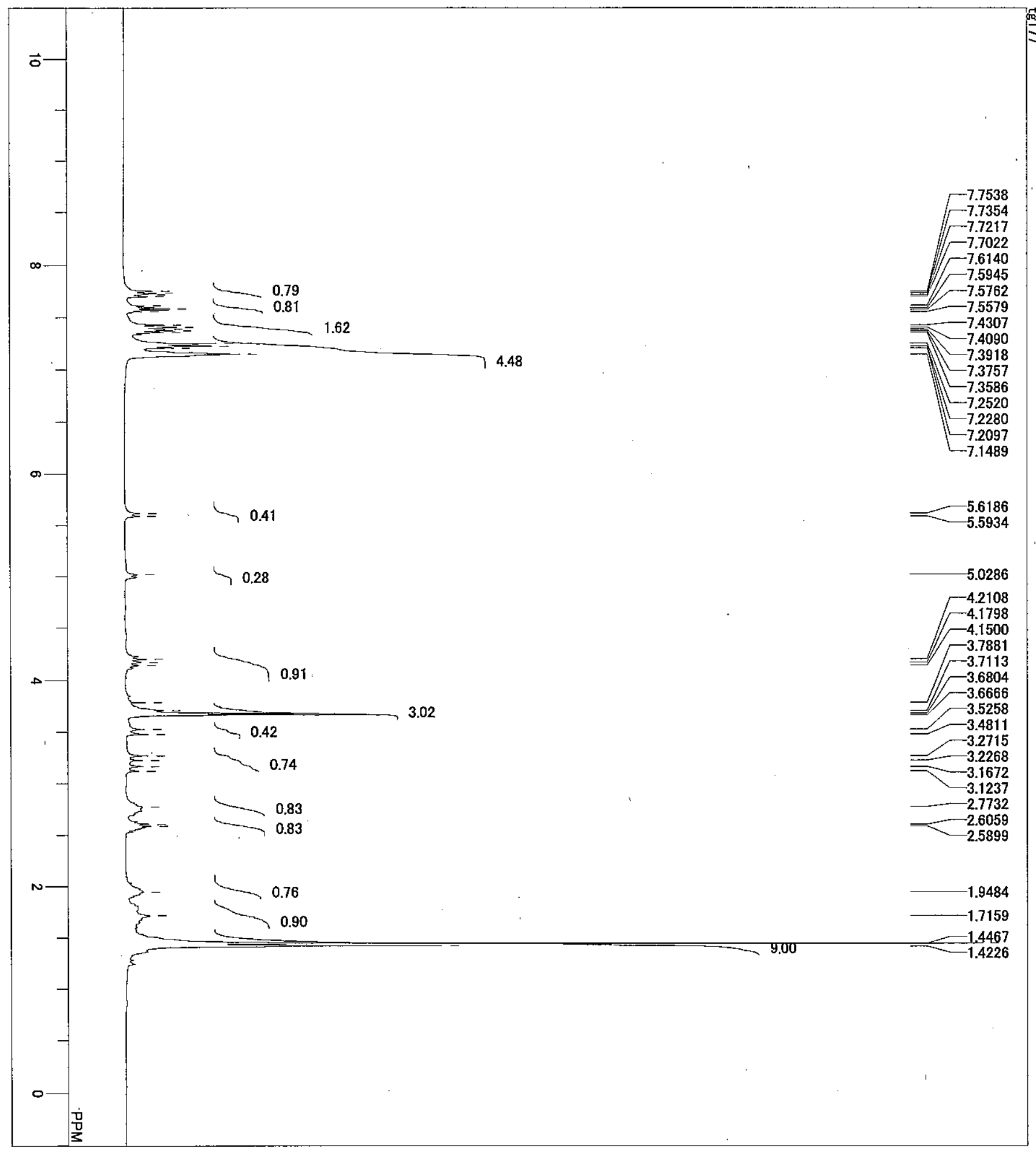

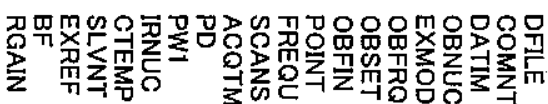<smiles>O=C1OC2(C=CC2)C(=O)C12Cc1ccccc1C2=O</smiles> 


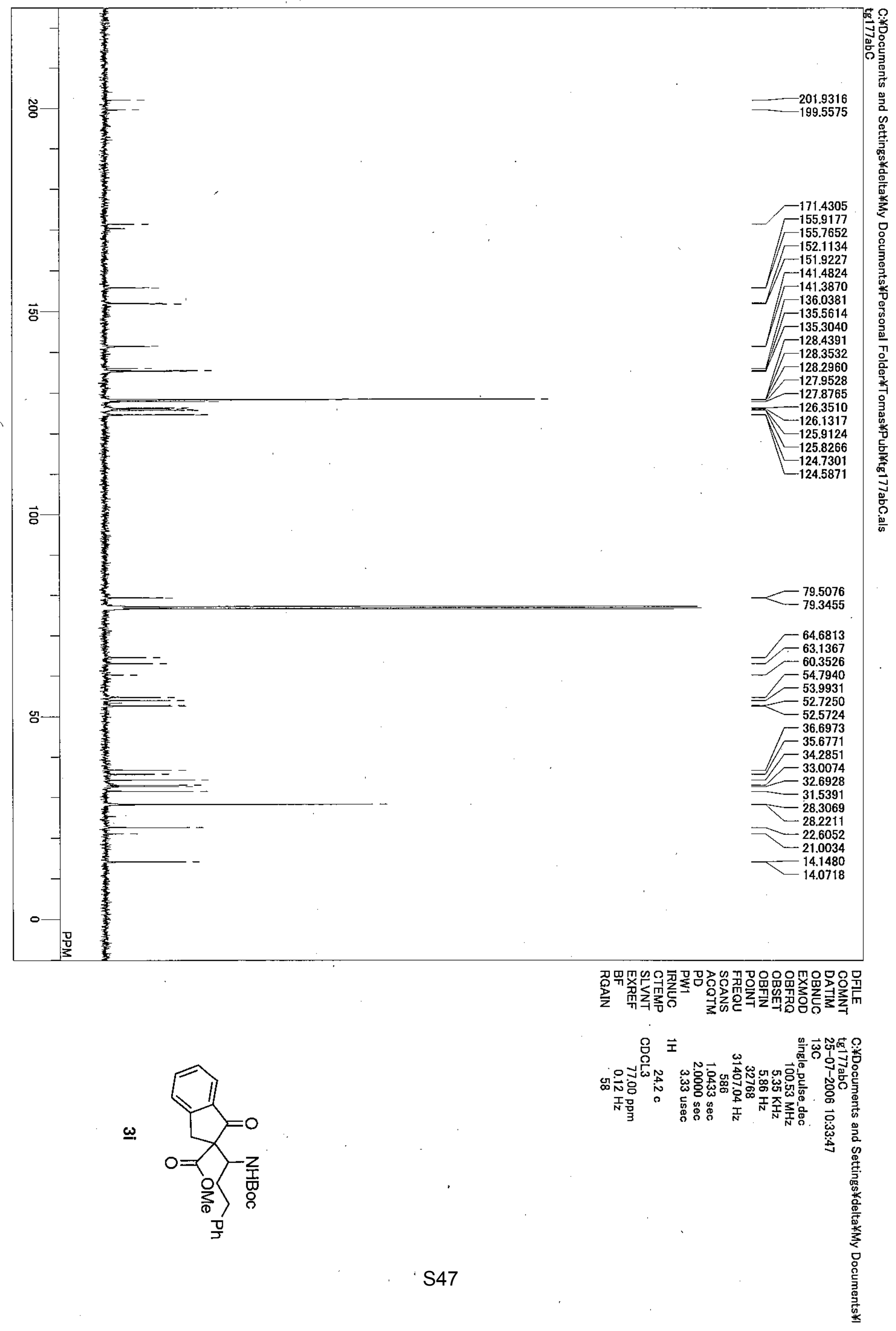




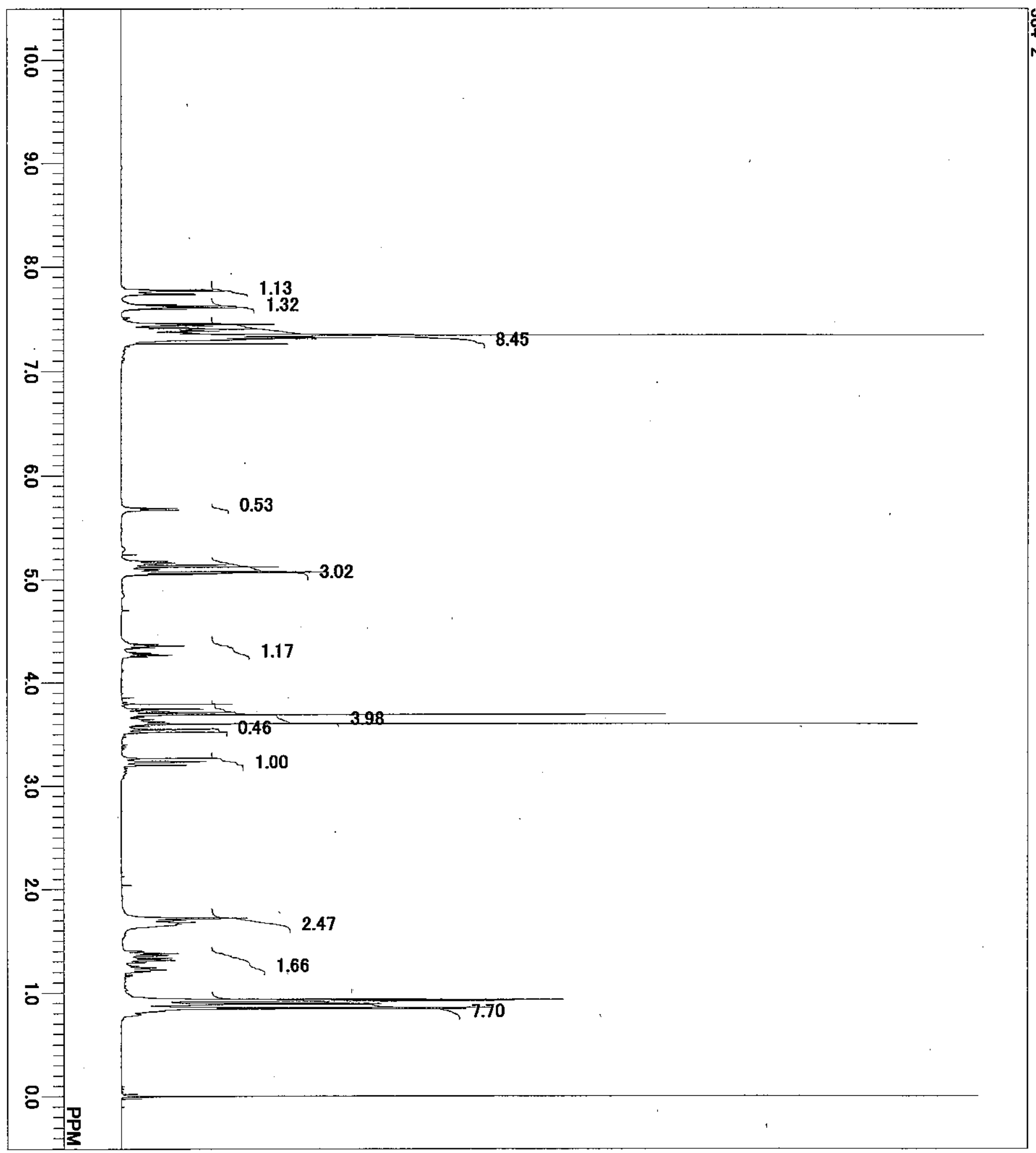

w

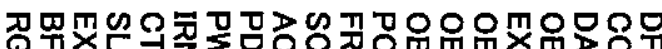

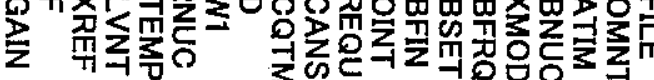

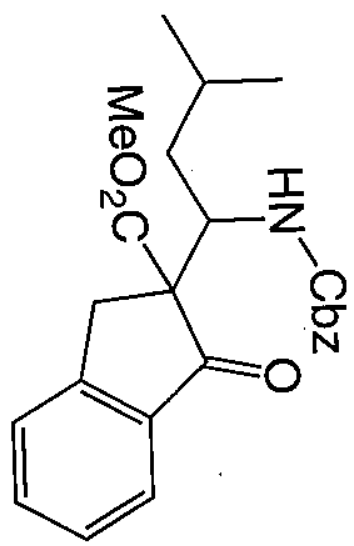

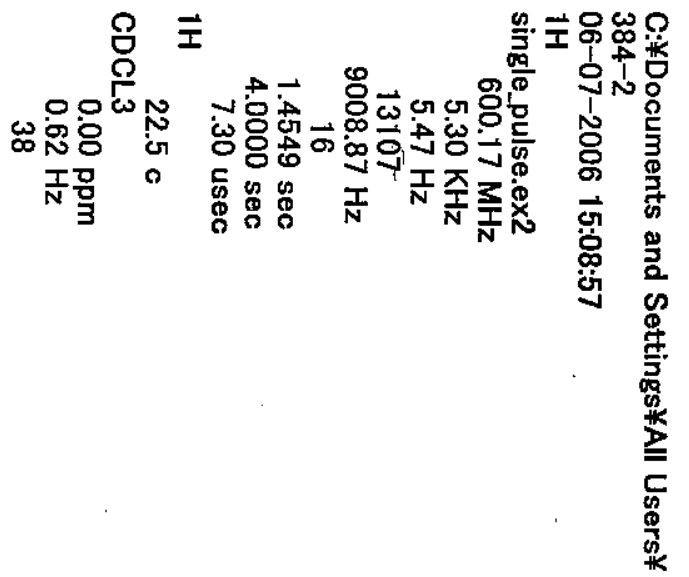




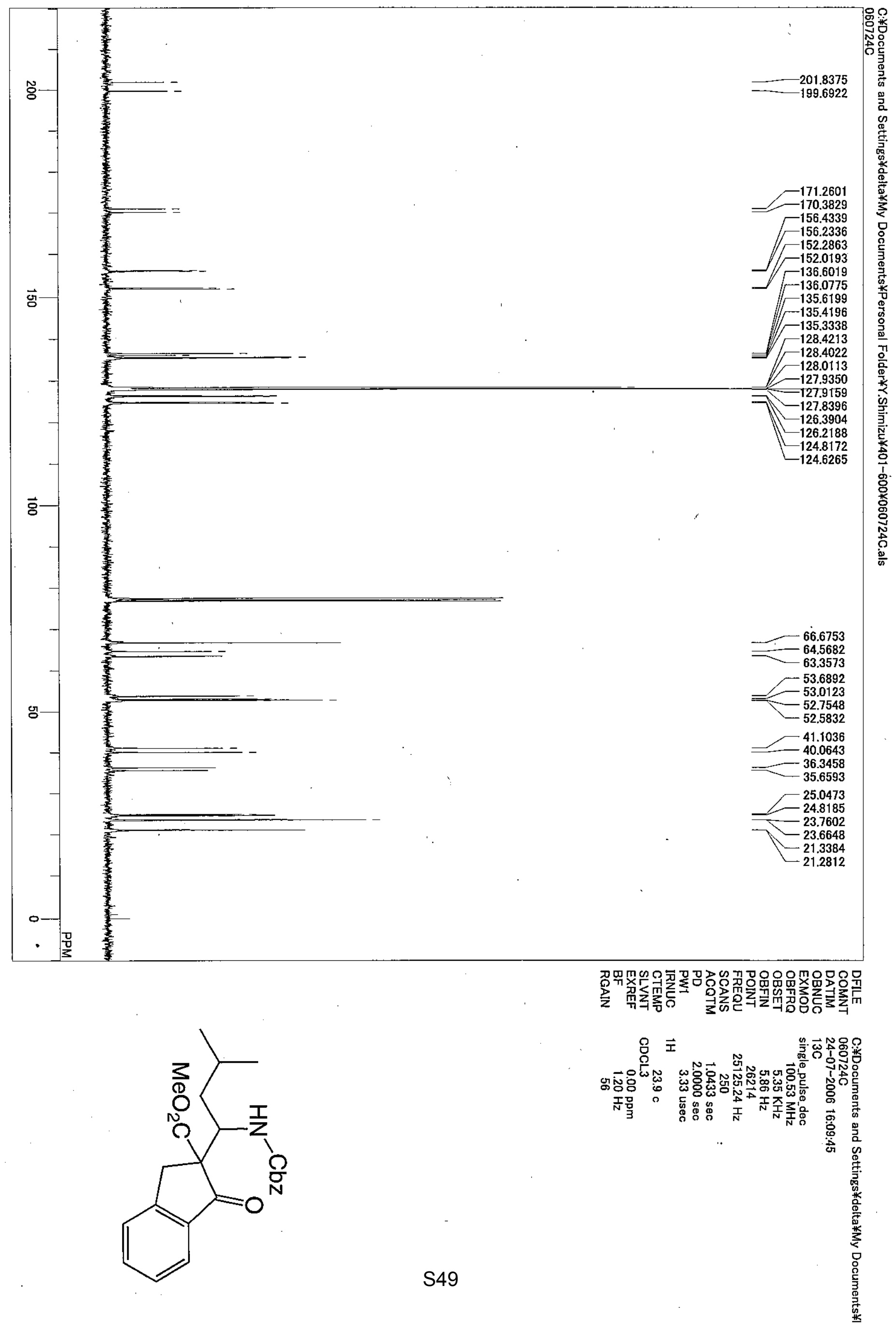




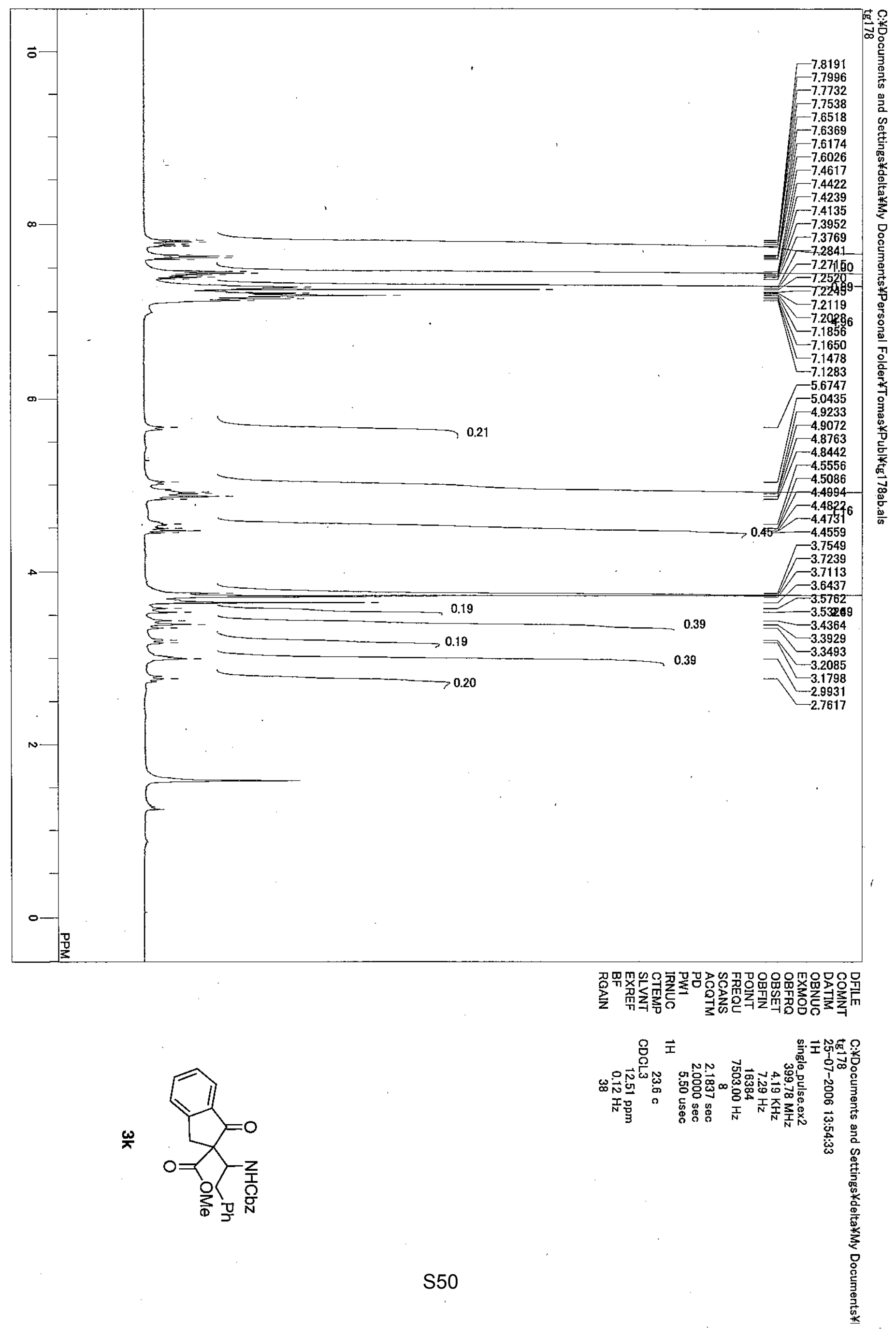




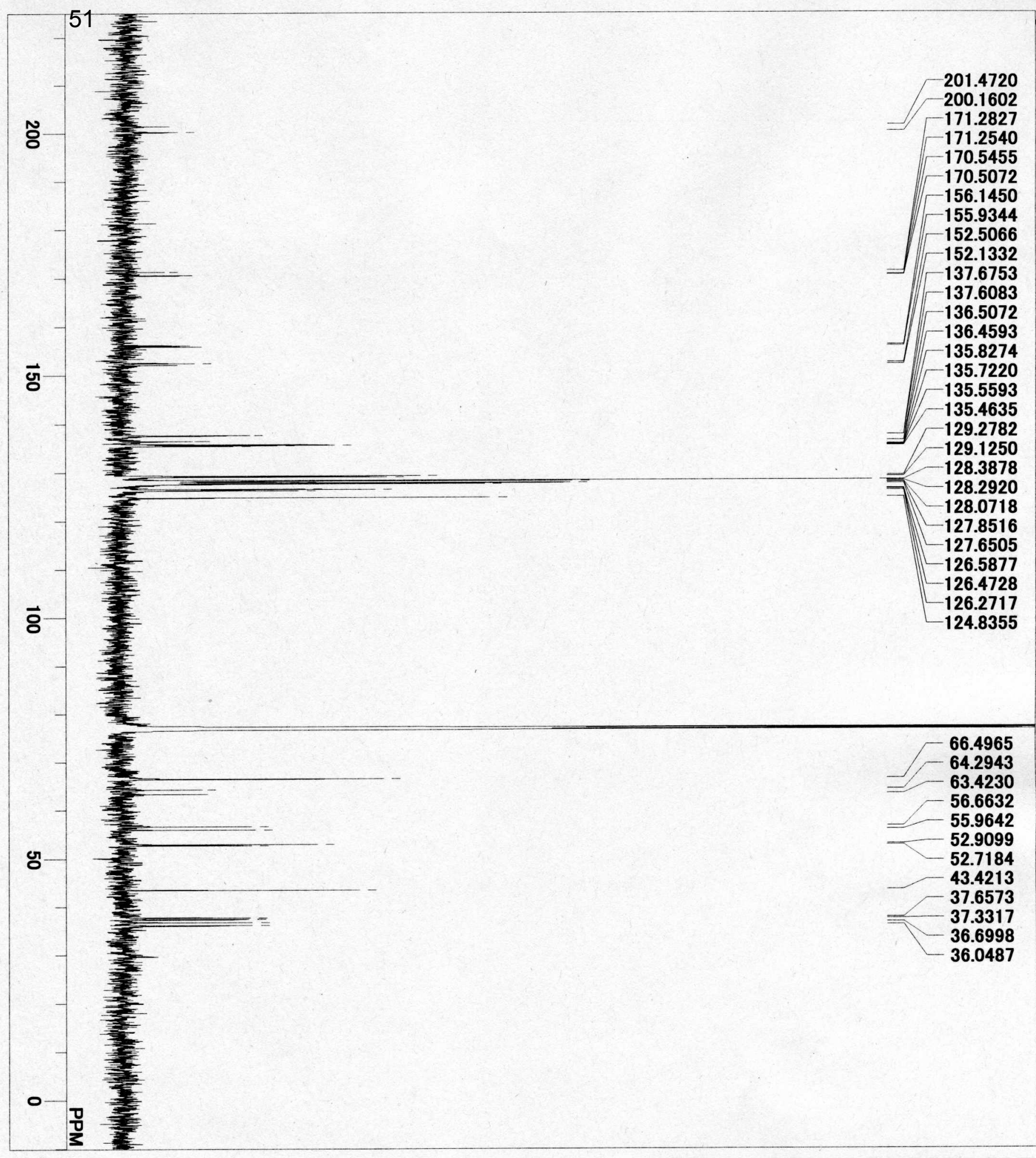

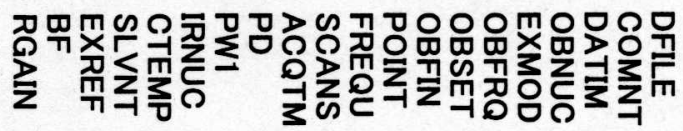<smiles>COC(CO)(CO)C1(O)Cc2ccccc2C1=O</smiles>

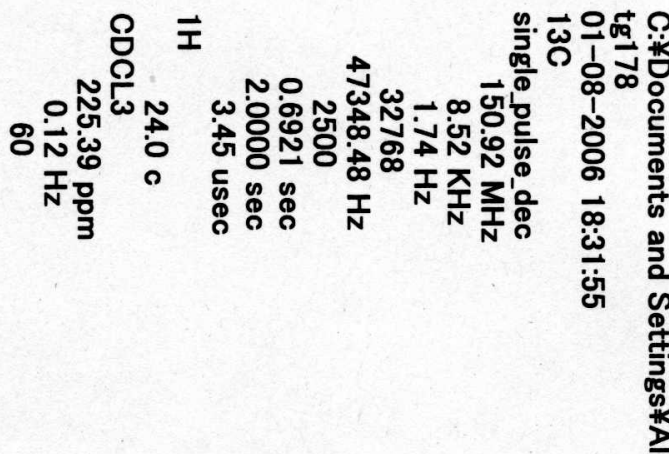




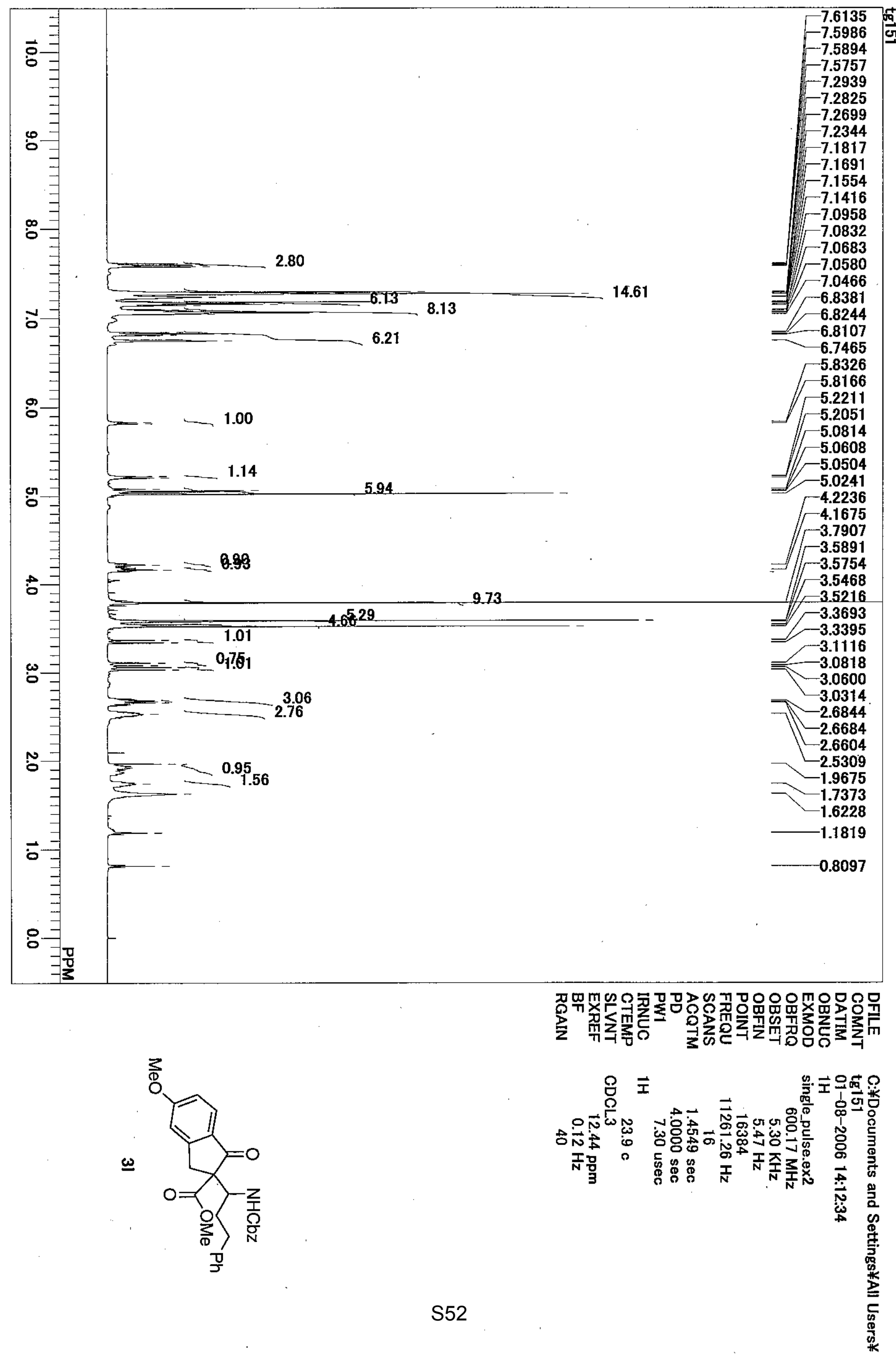




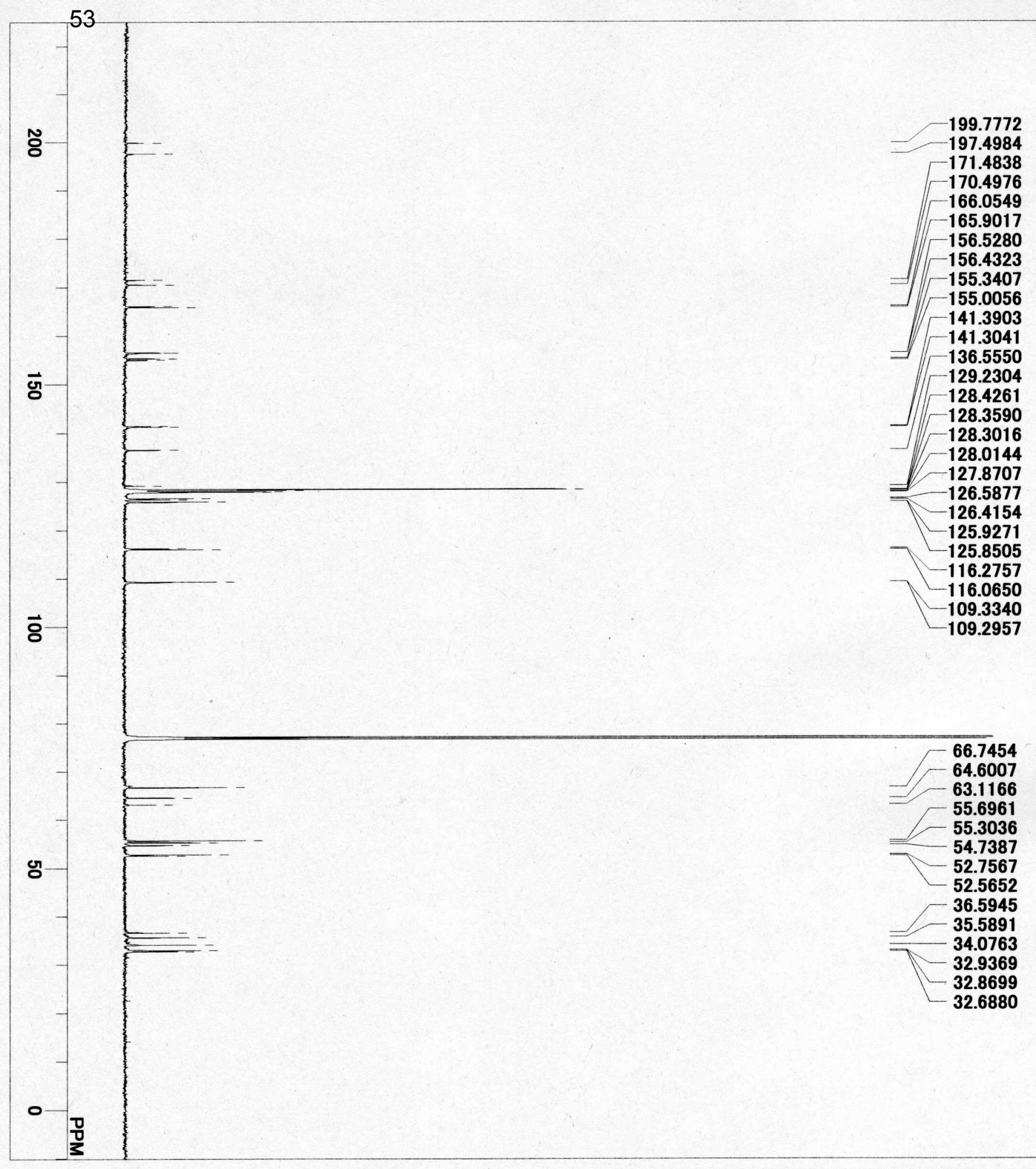<smiles>CCC1(C(C)C)Cc2cc(OC)ccc2C1=O</smiles> 


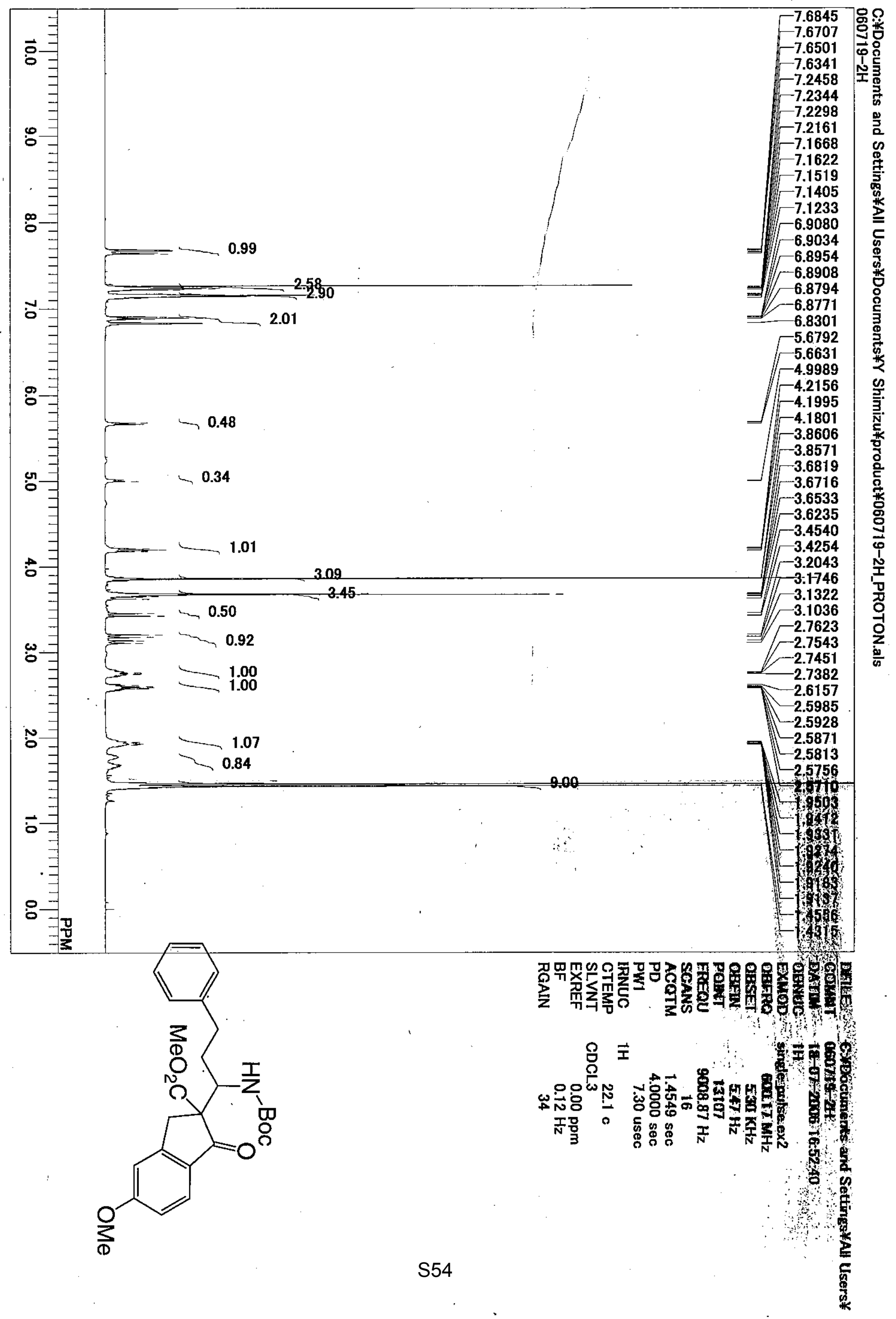




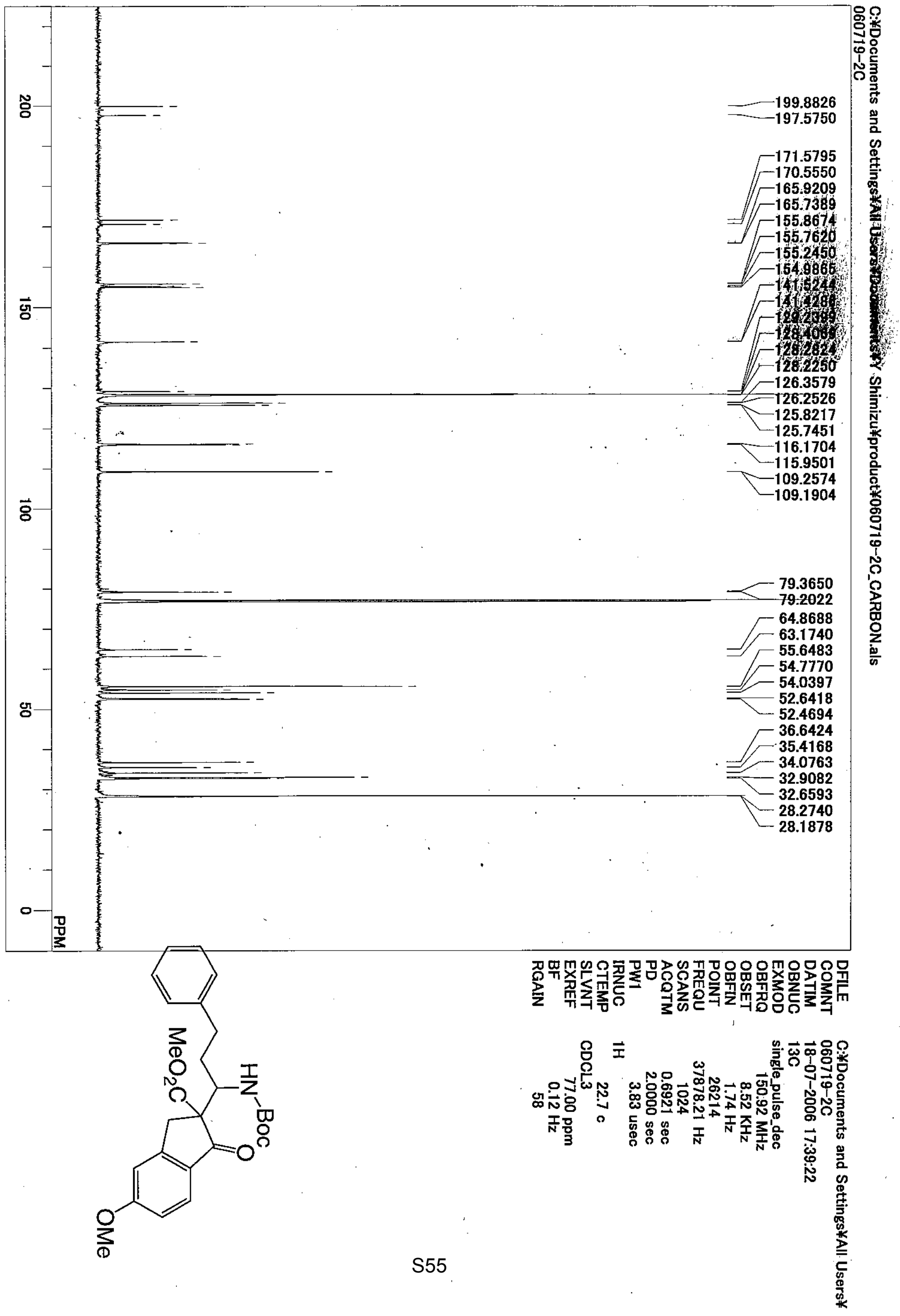




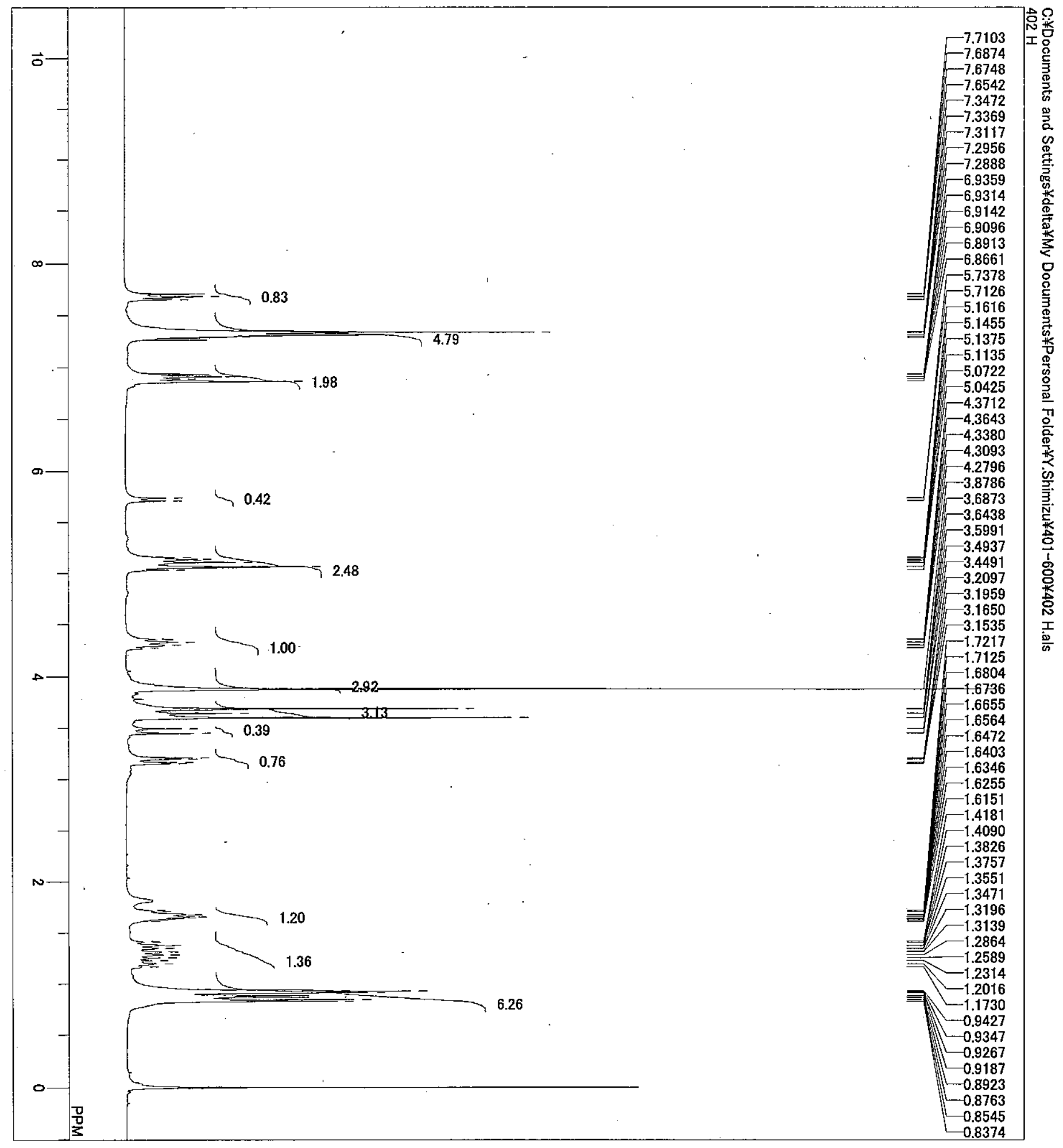

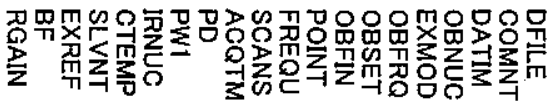

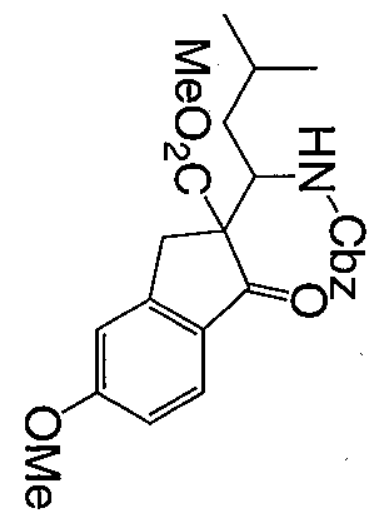

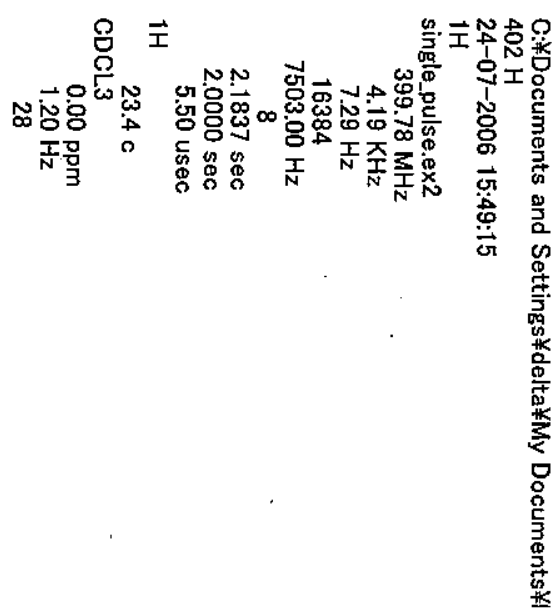




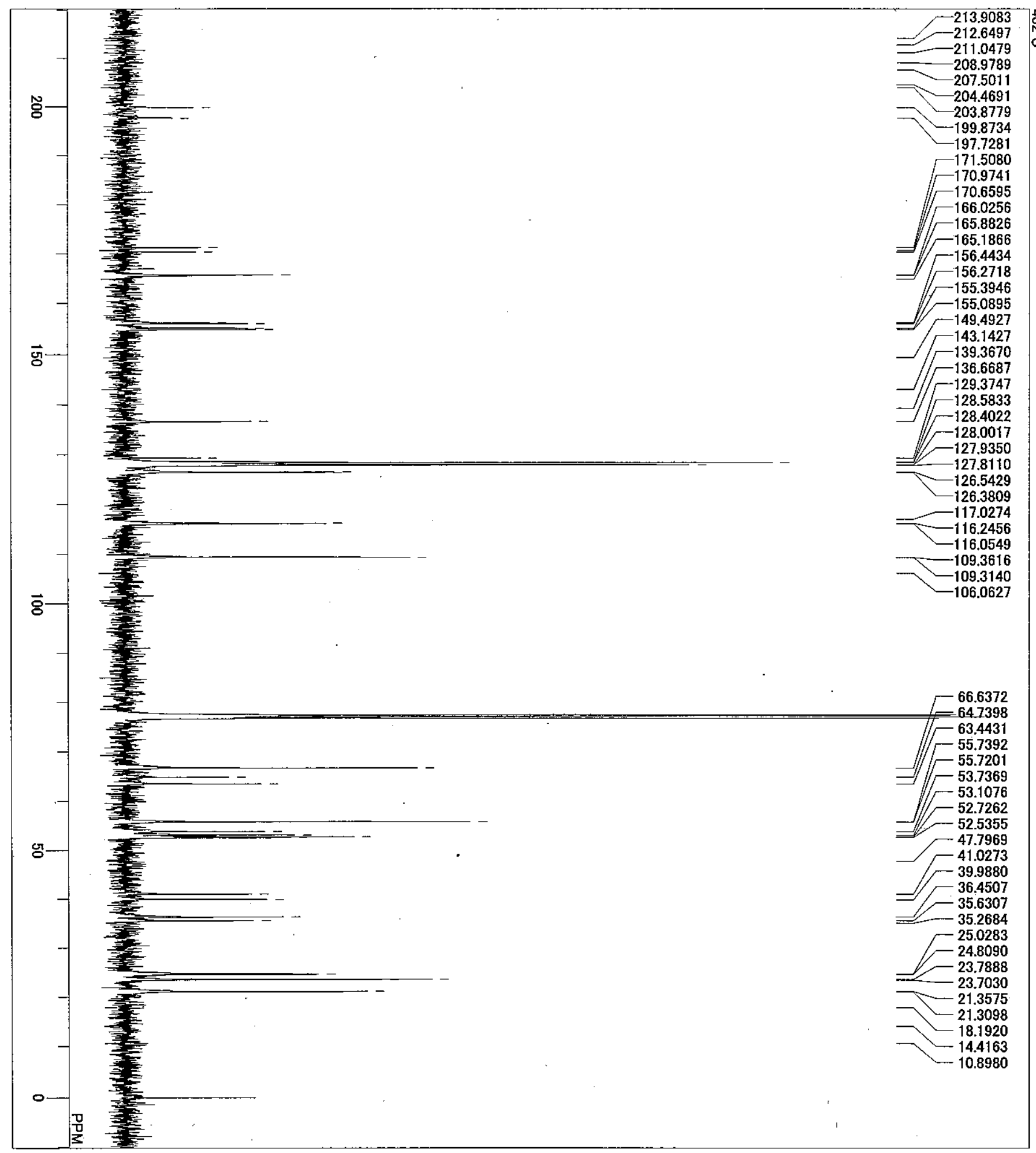

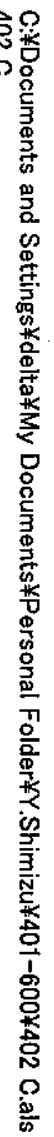

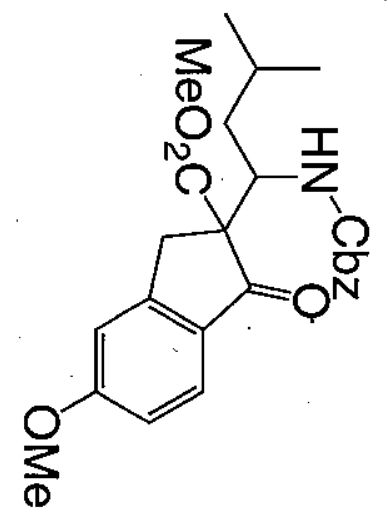

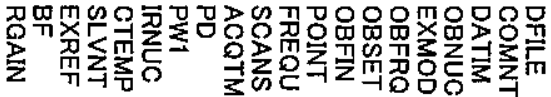

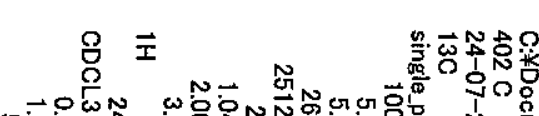
m

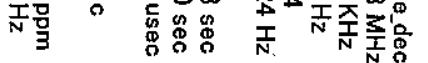




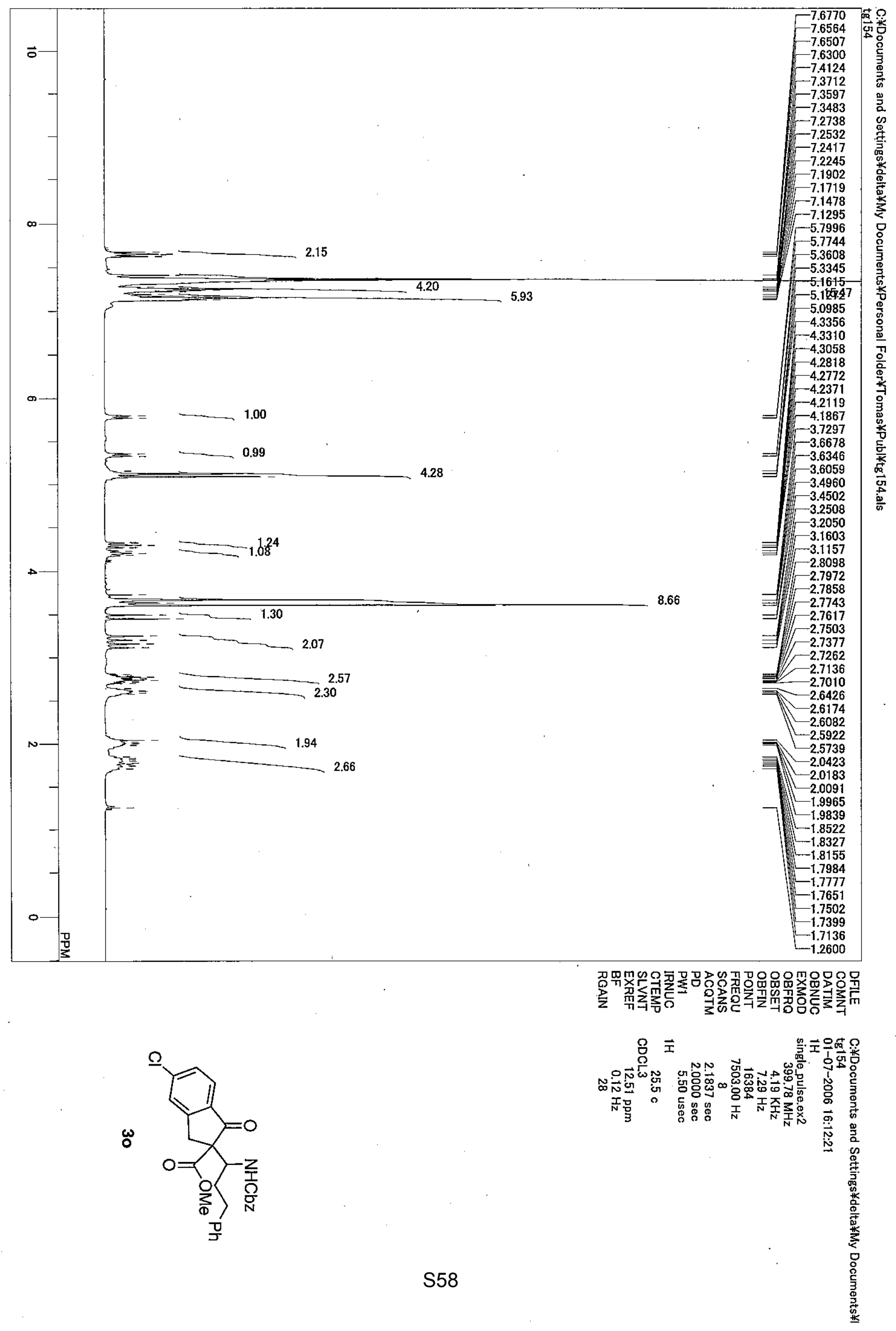




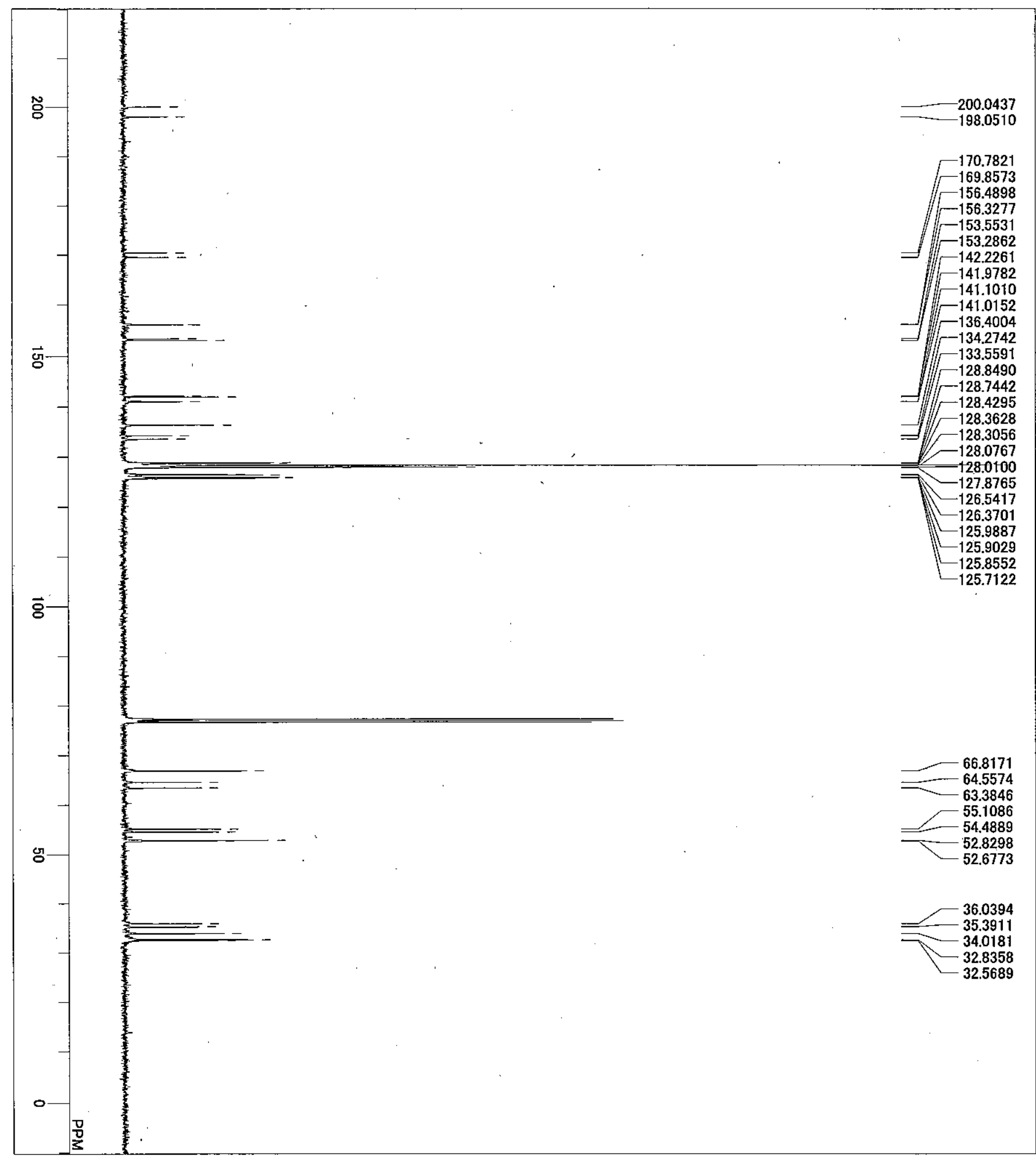

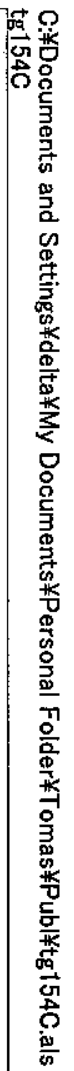

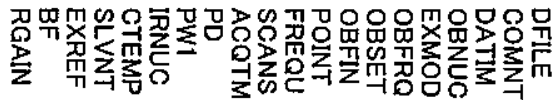
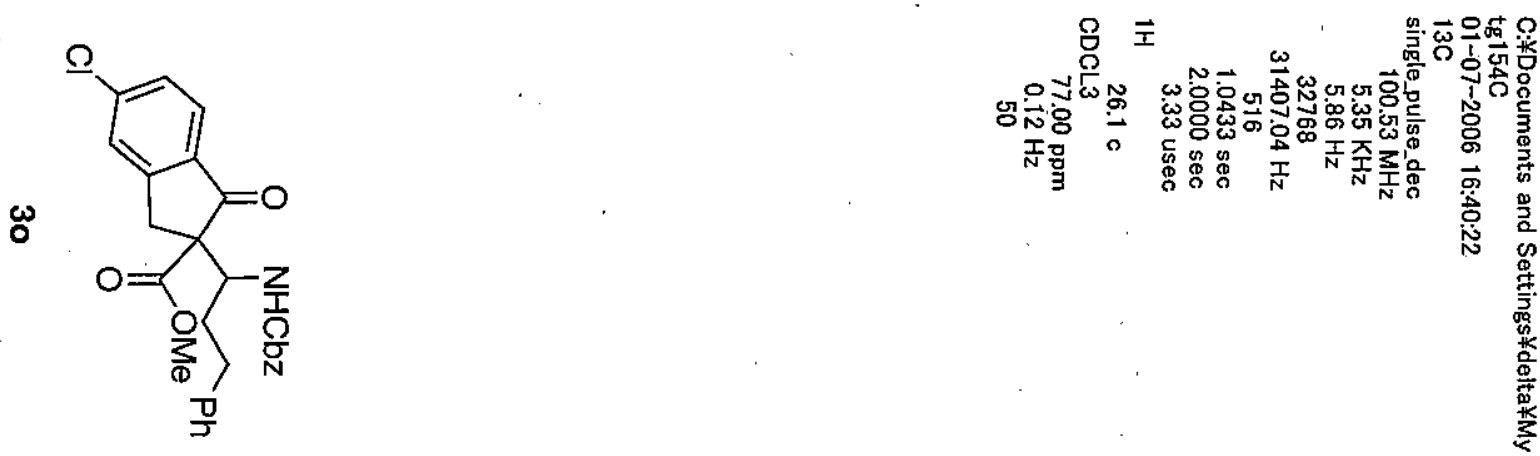


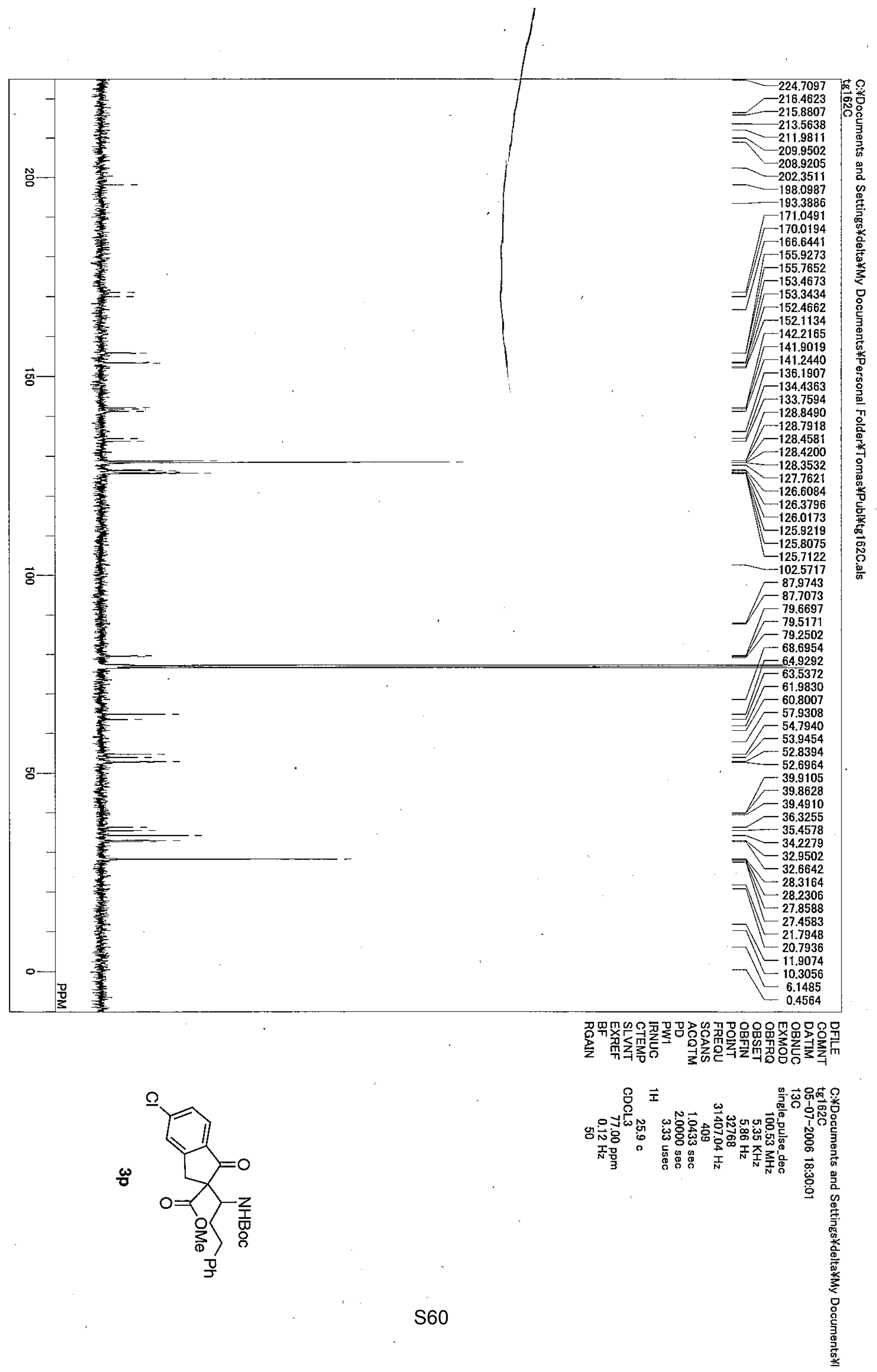




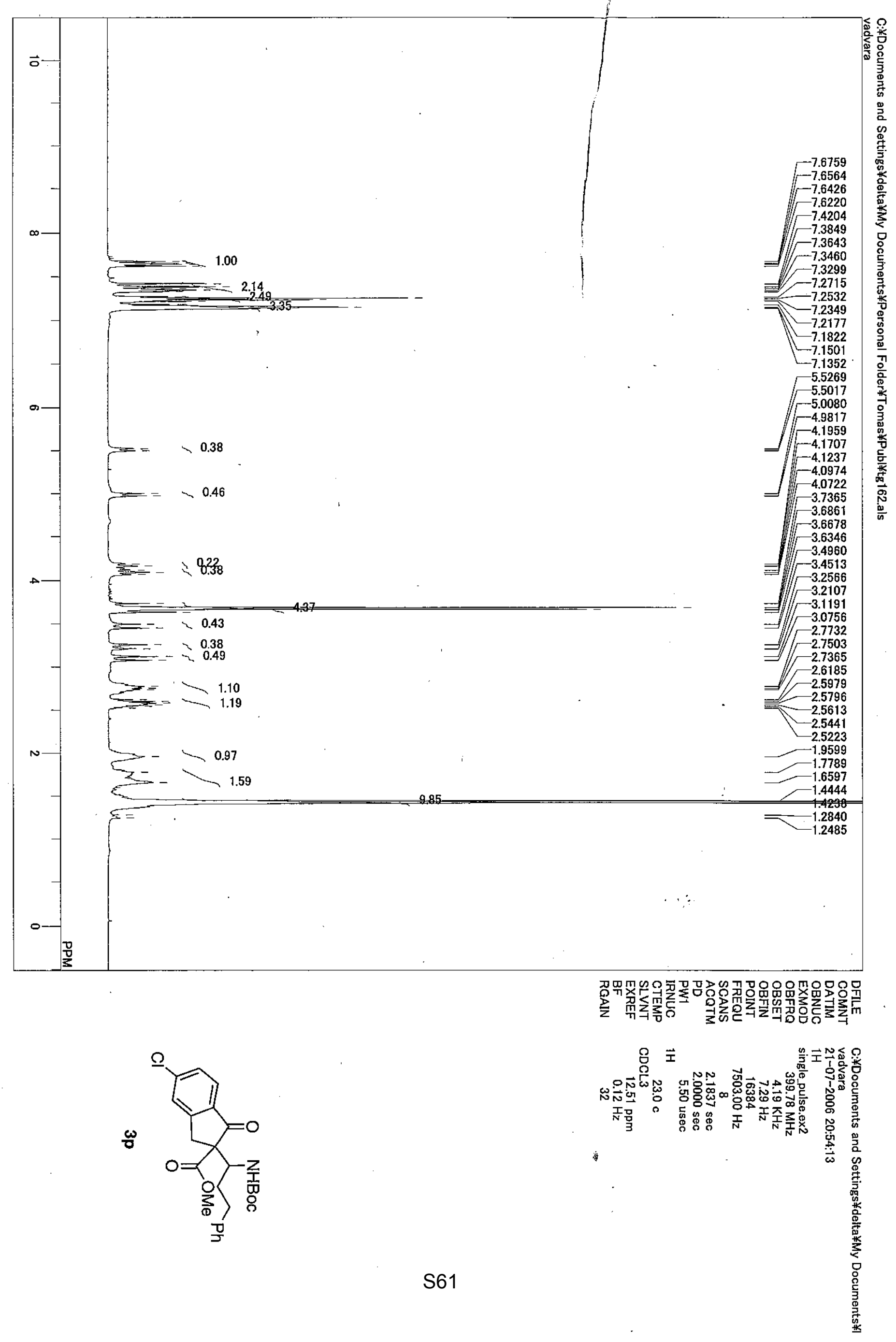




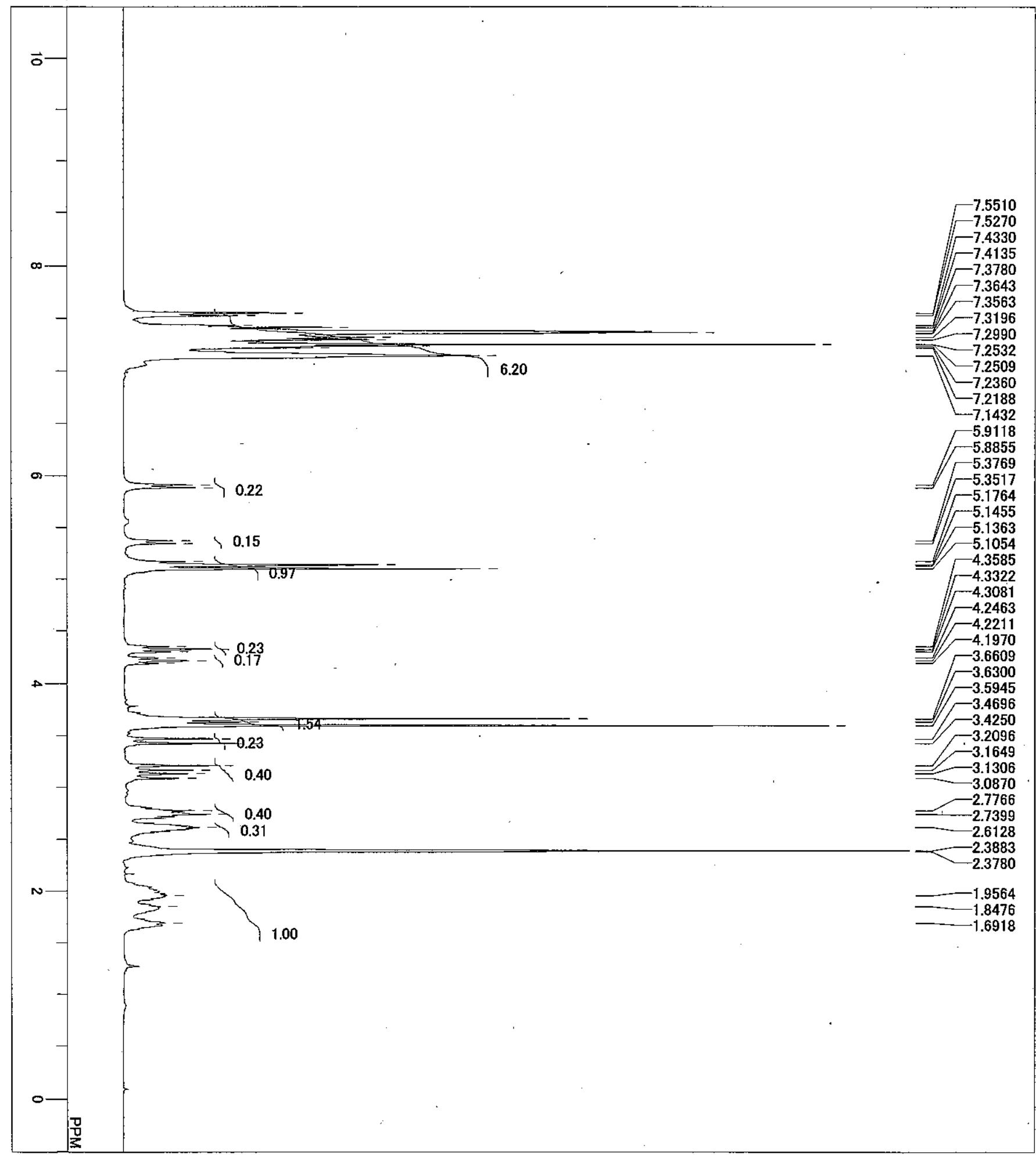

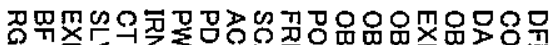

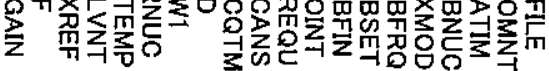

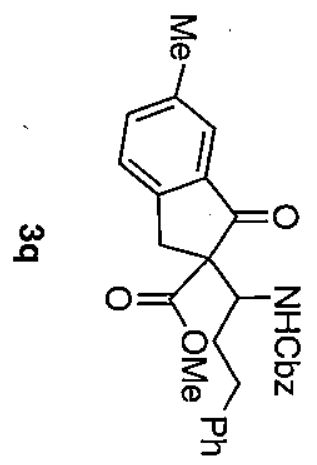

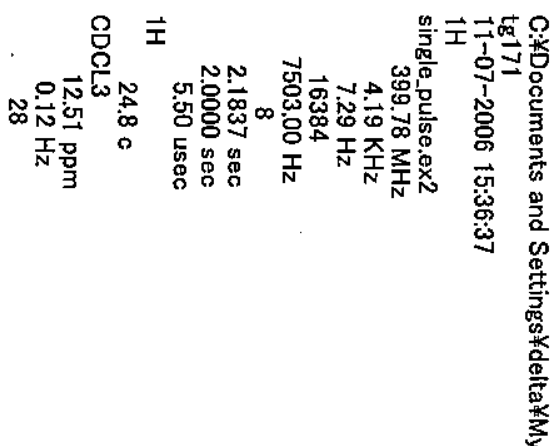




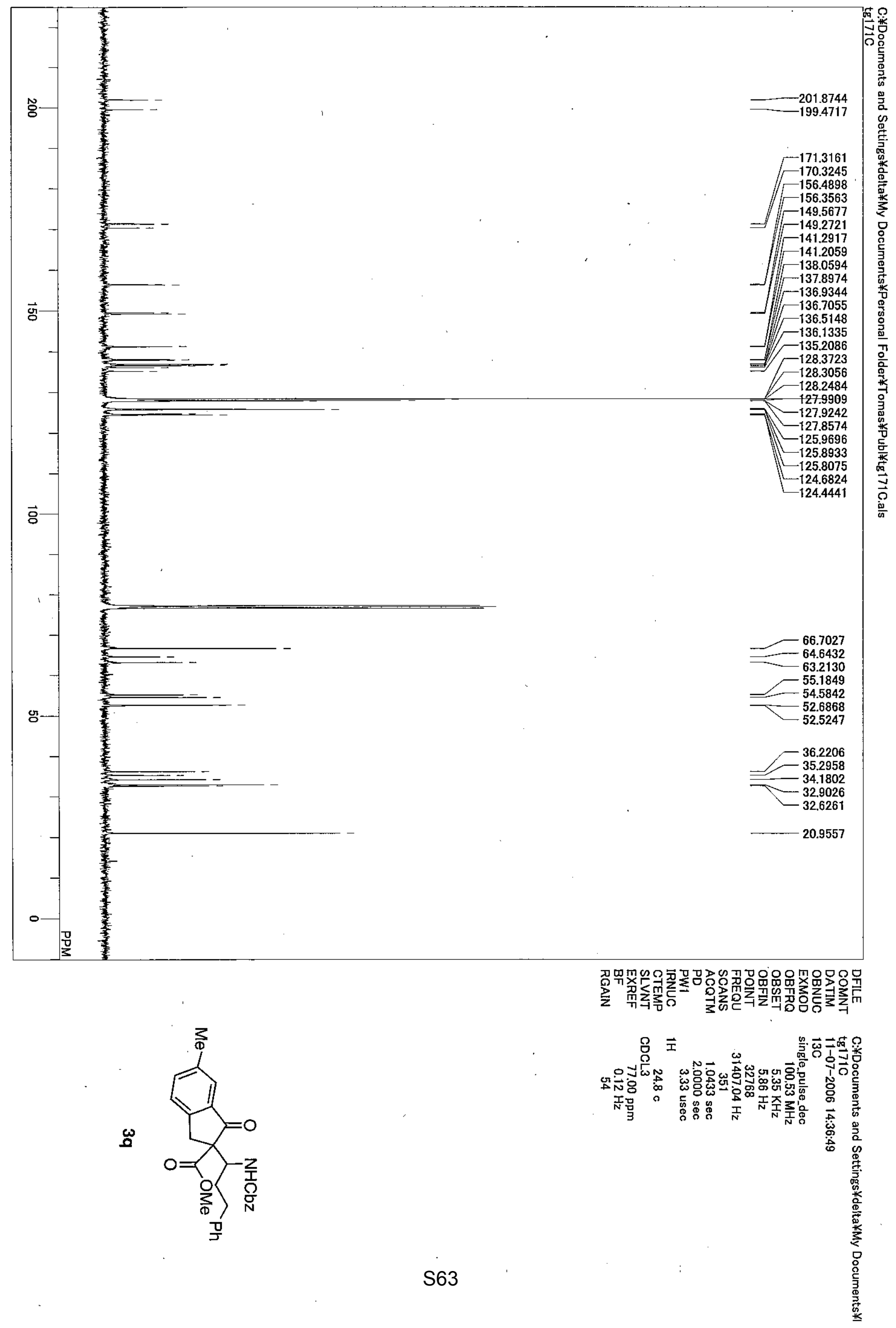




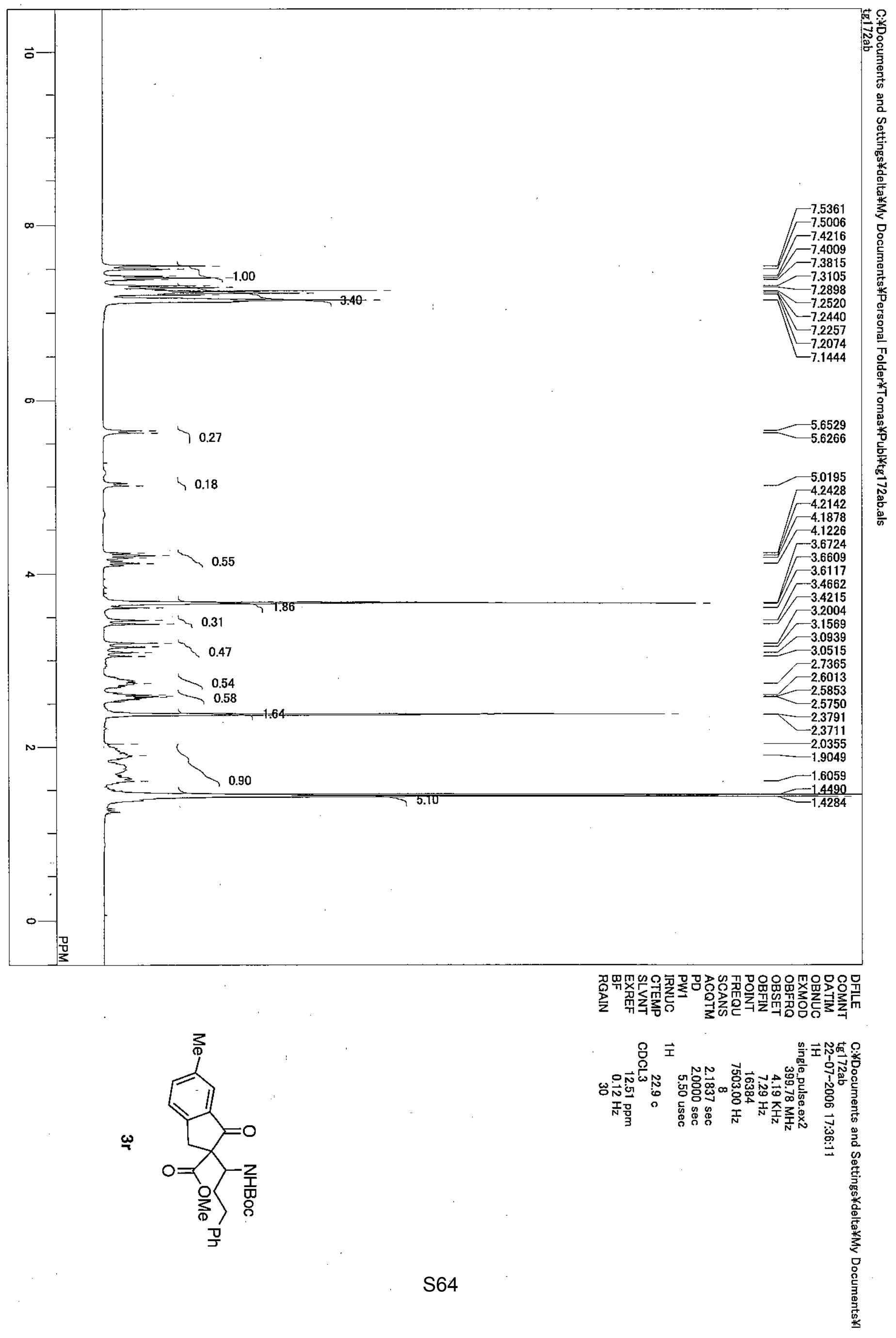




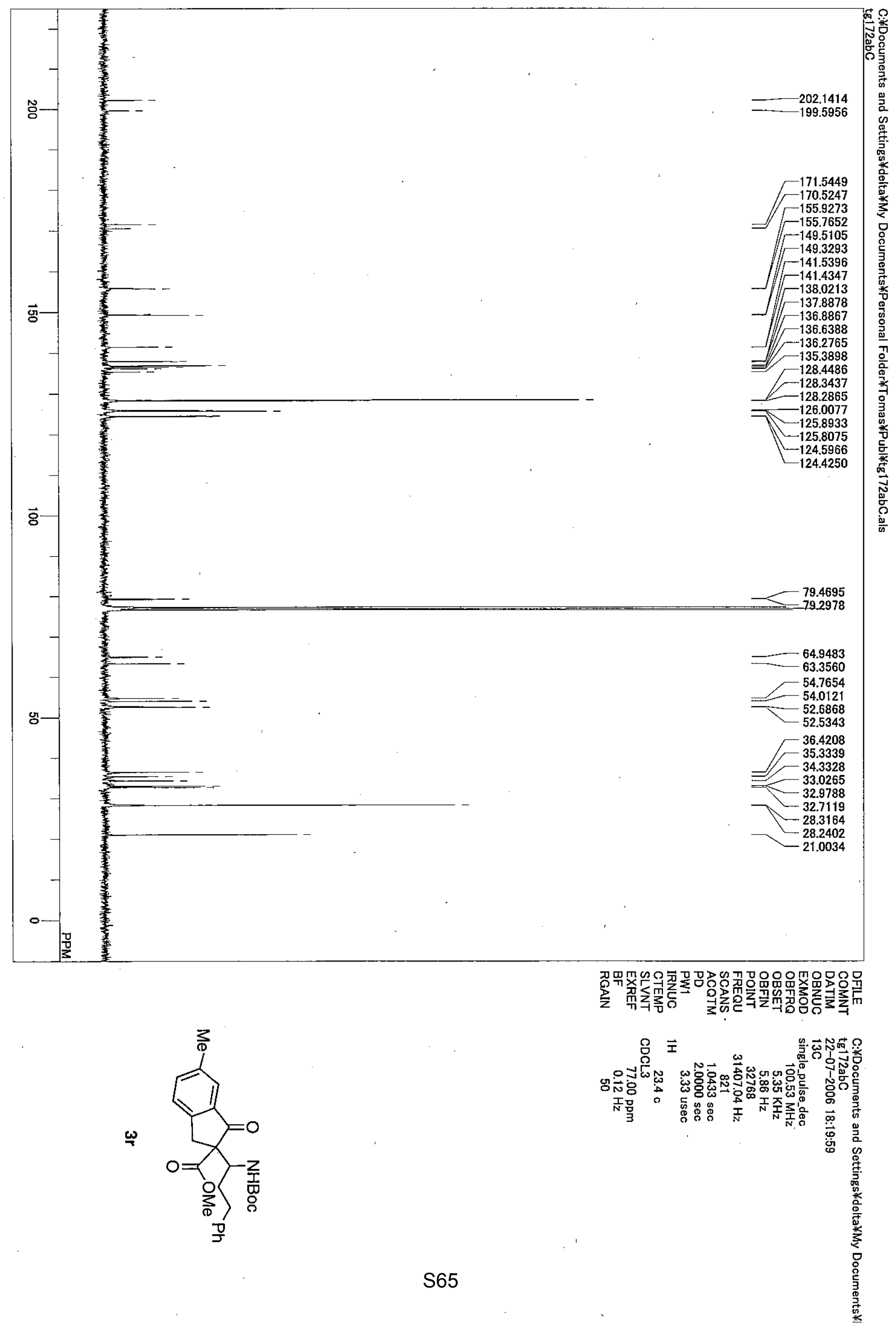




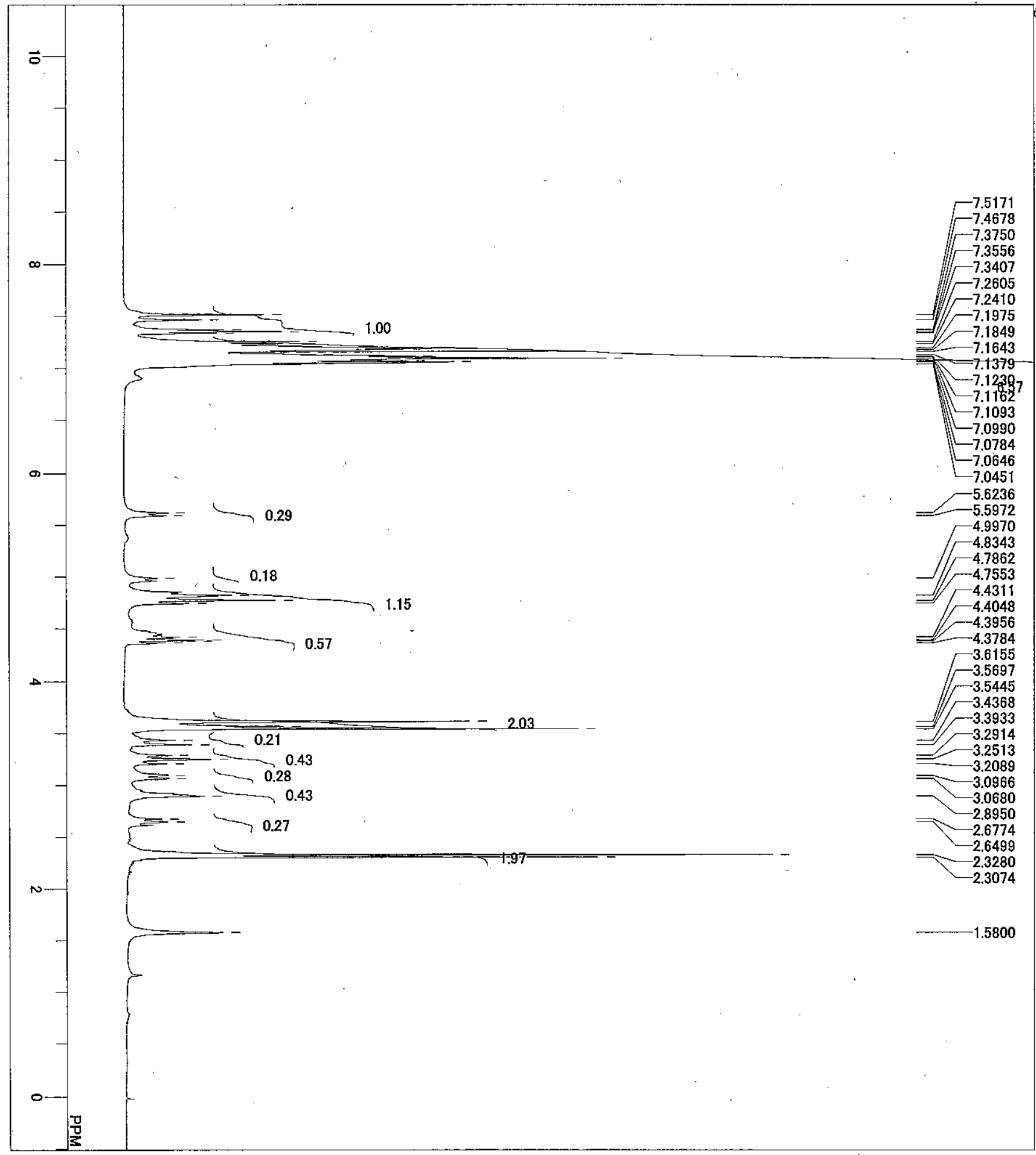

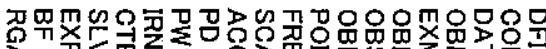

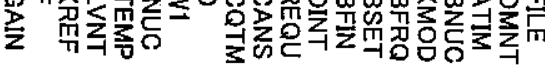

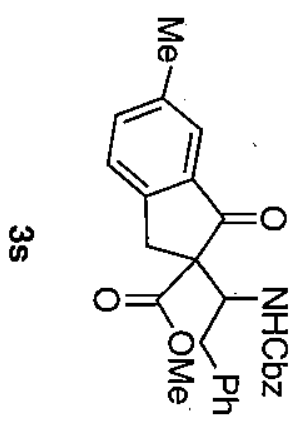


67

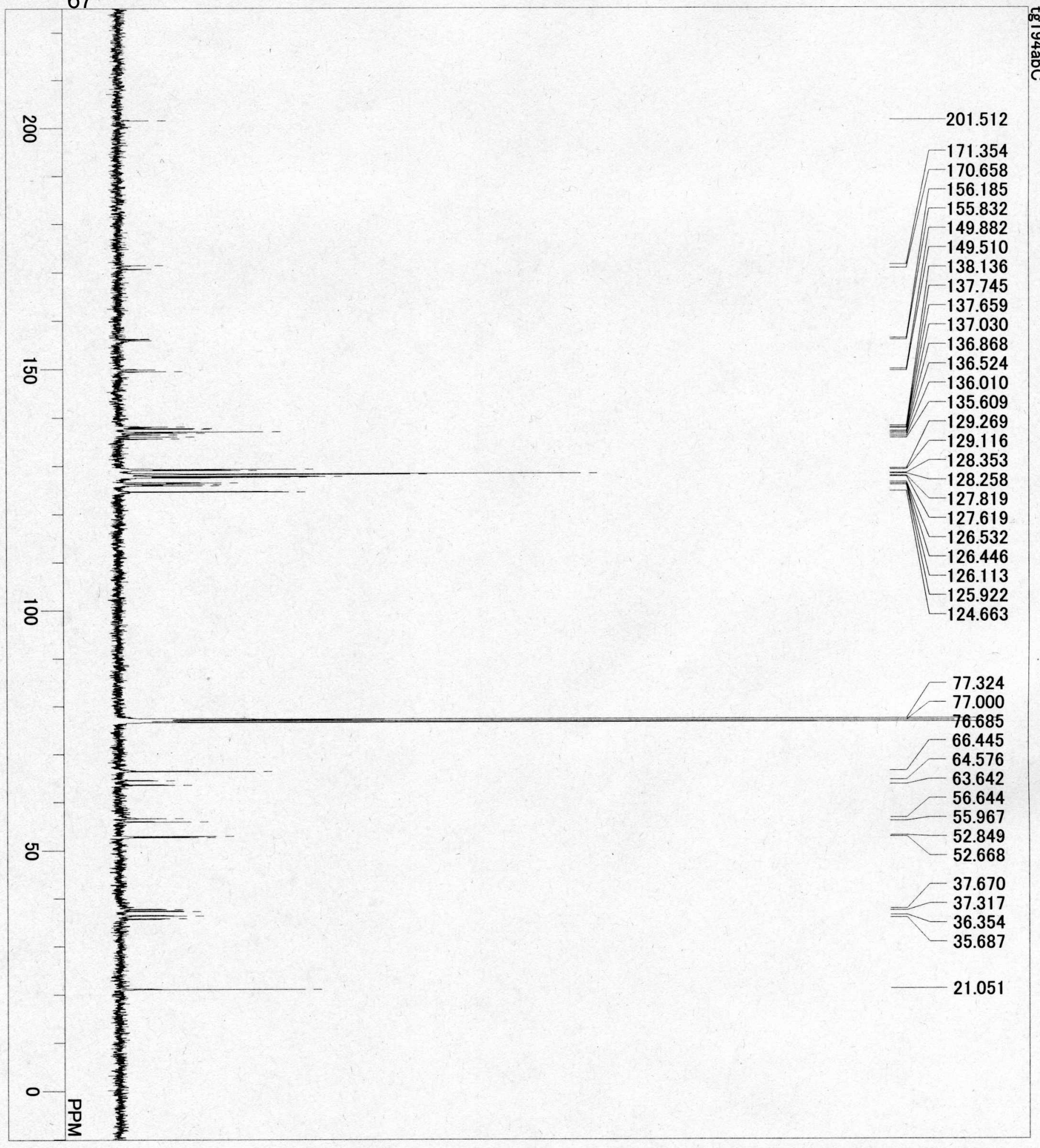

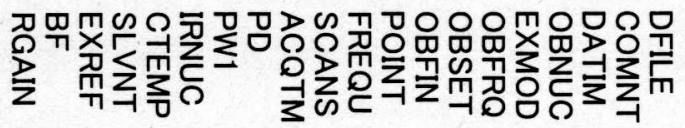
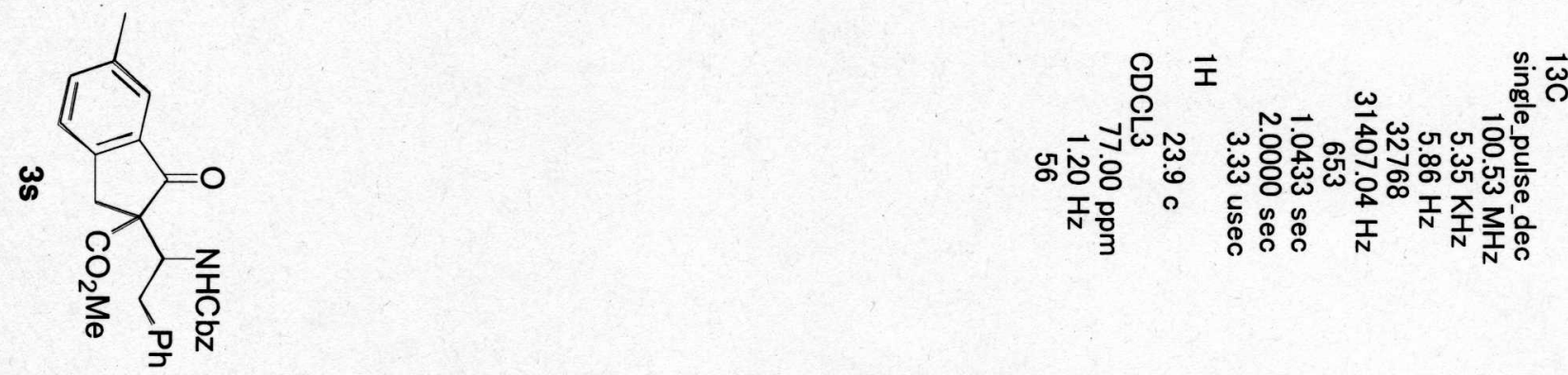

号.

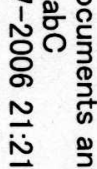

$\underset{\overrightarrow{\dot{\vec{N}}}}{\overrightarrow{\mathrm{N}}}$ 

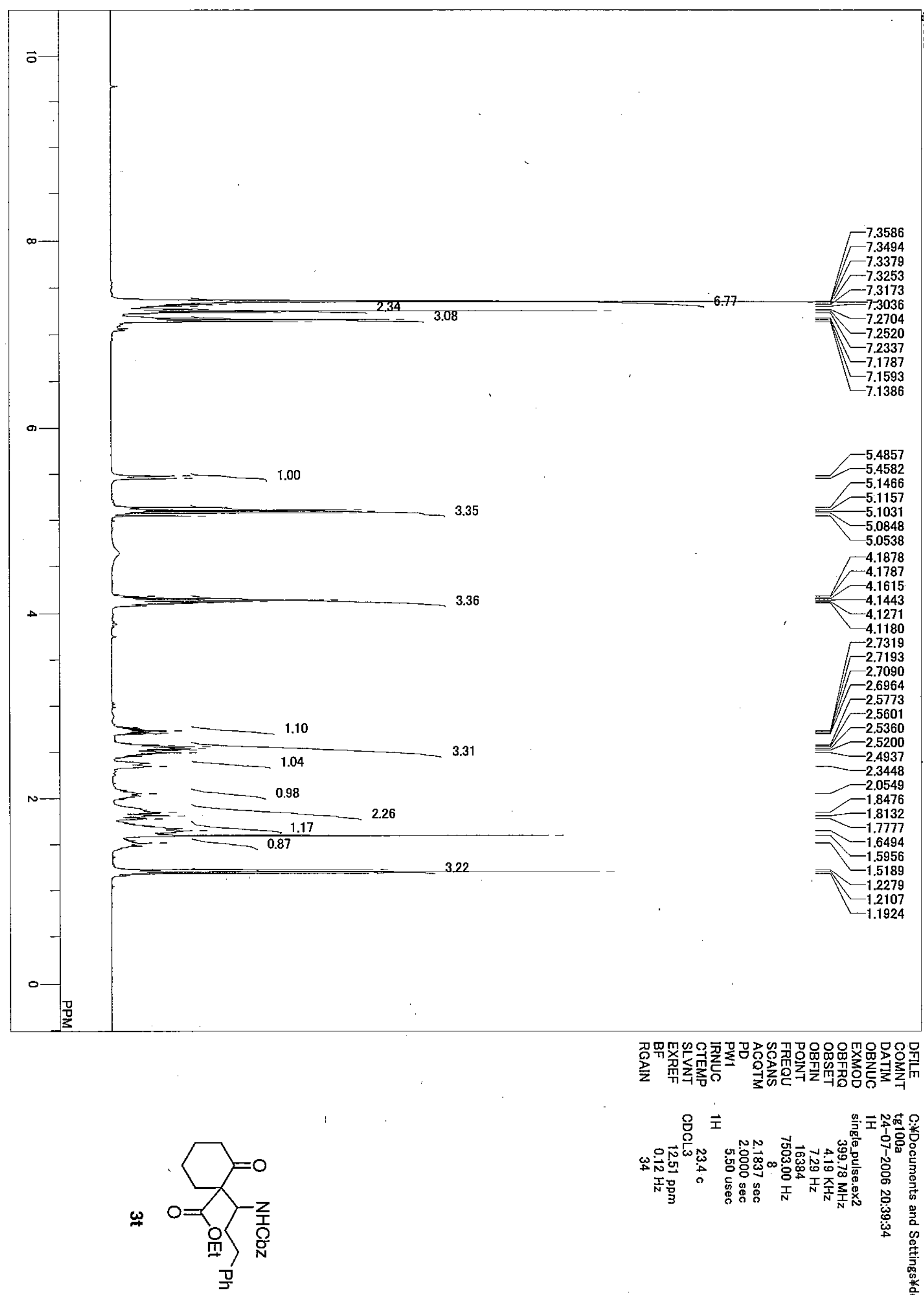


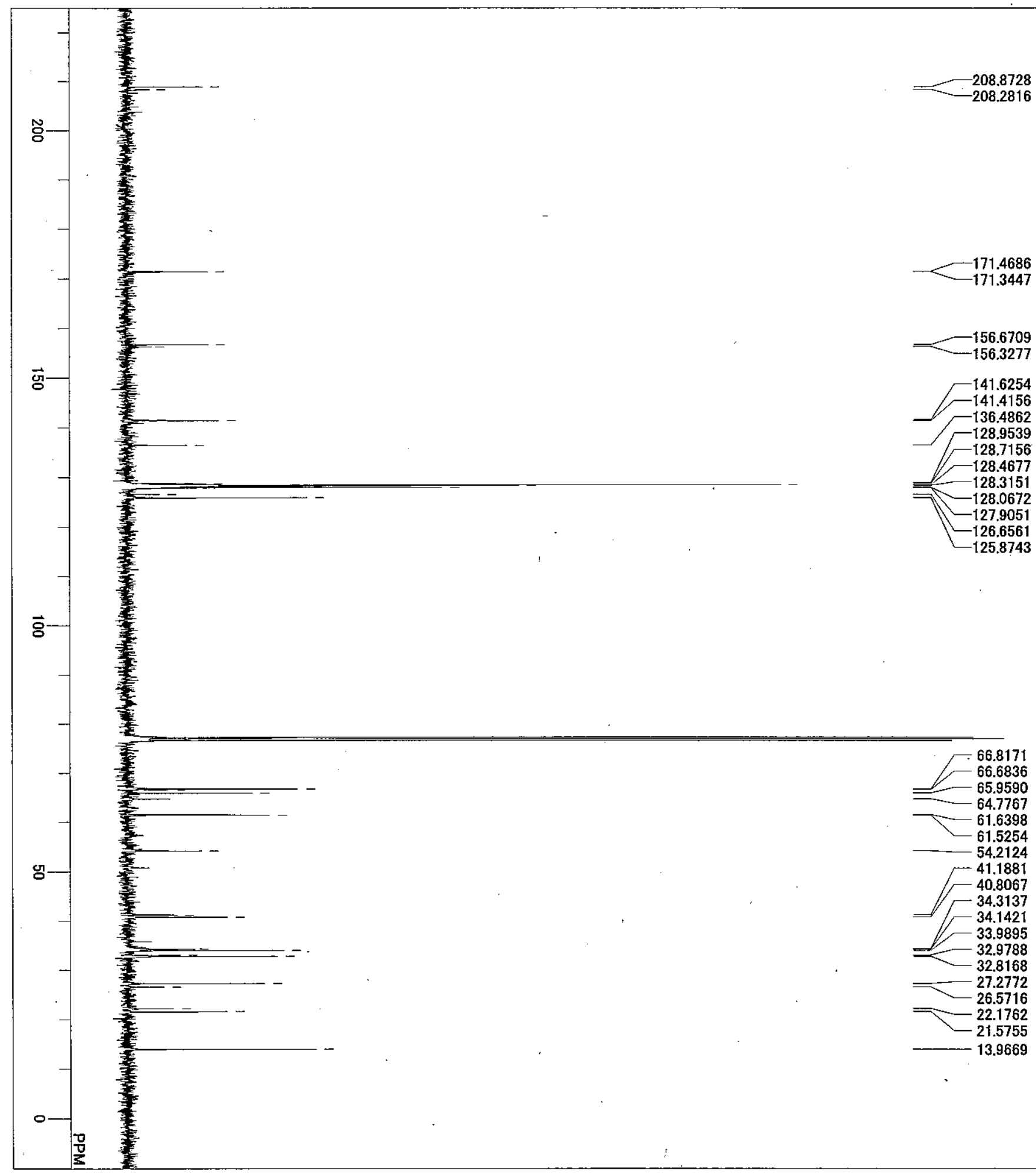

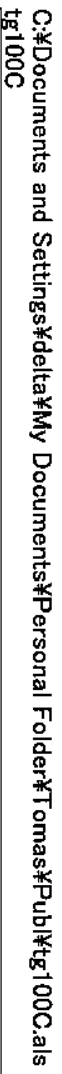

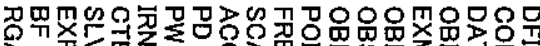

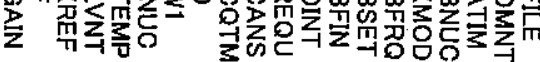<smiles>N#CCC(C#N)C1(C(=O)O)CCCCC1=O</smiles> 


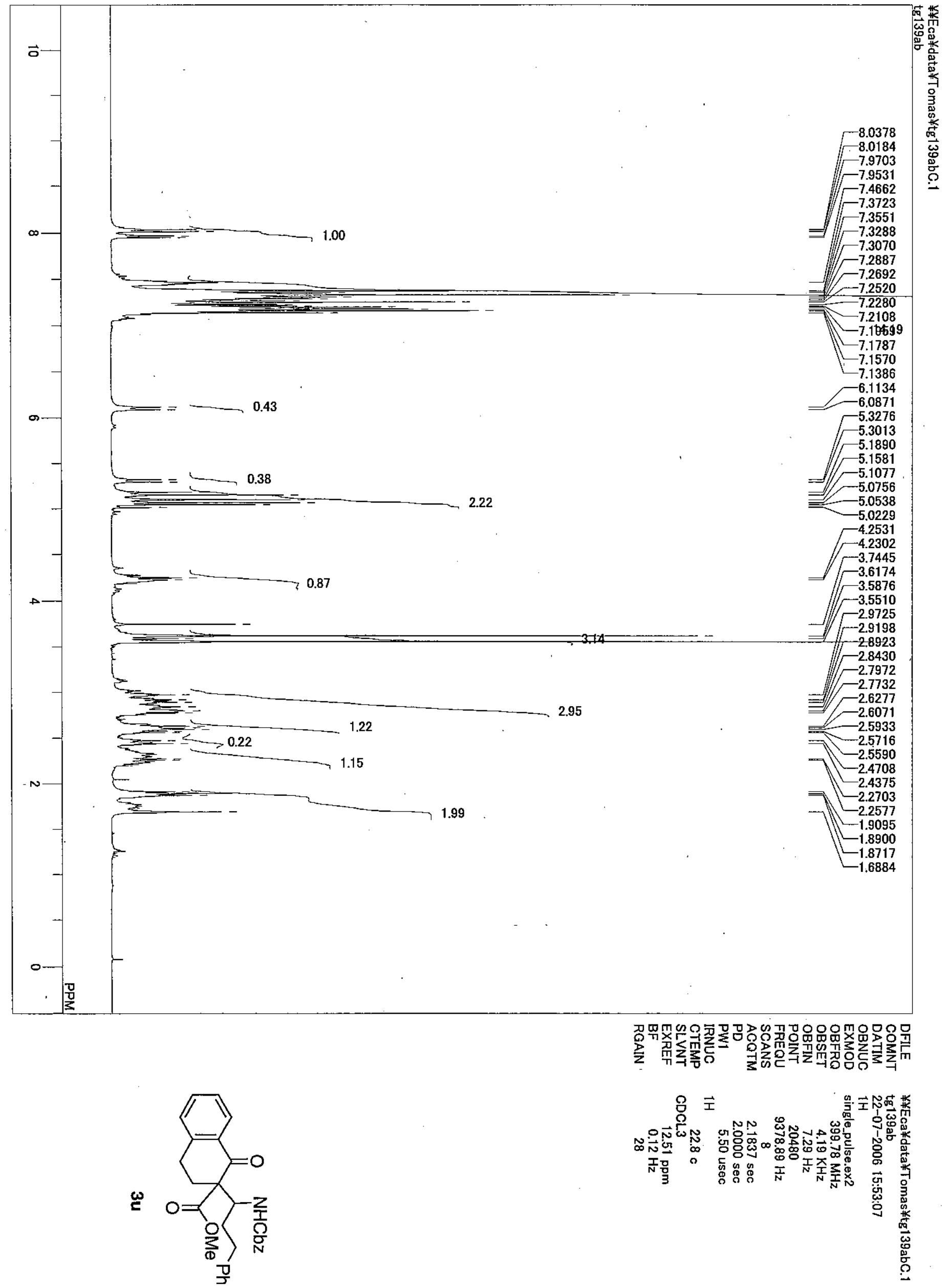




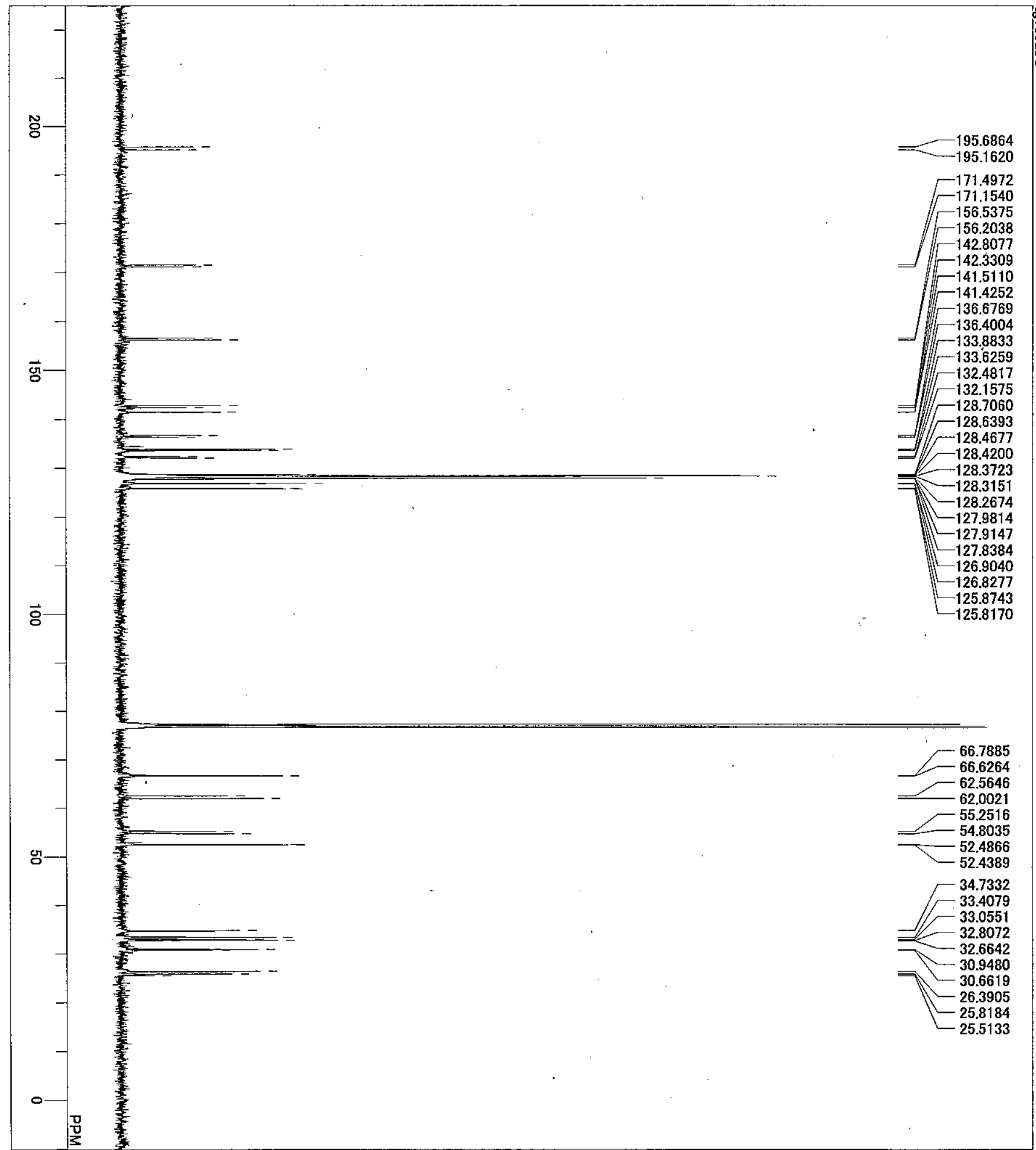

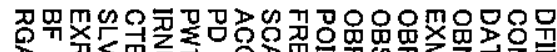

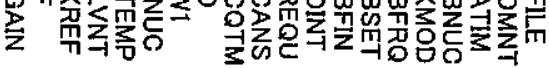

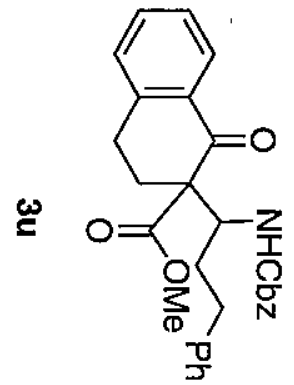

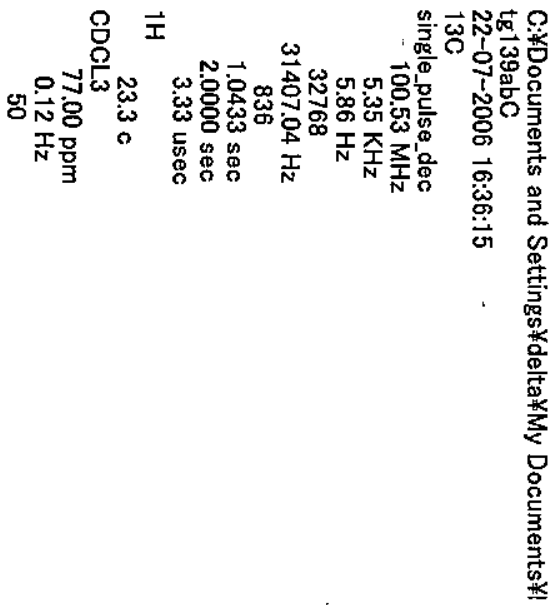




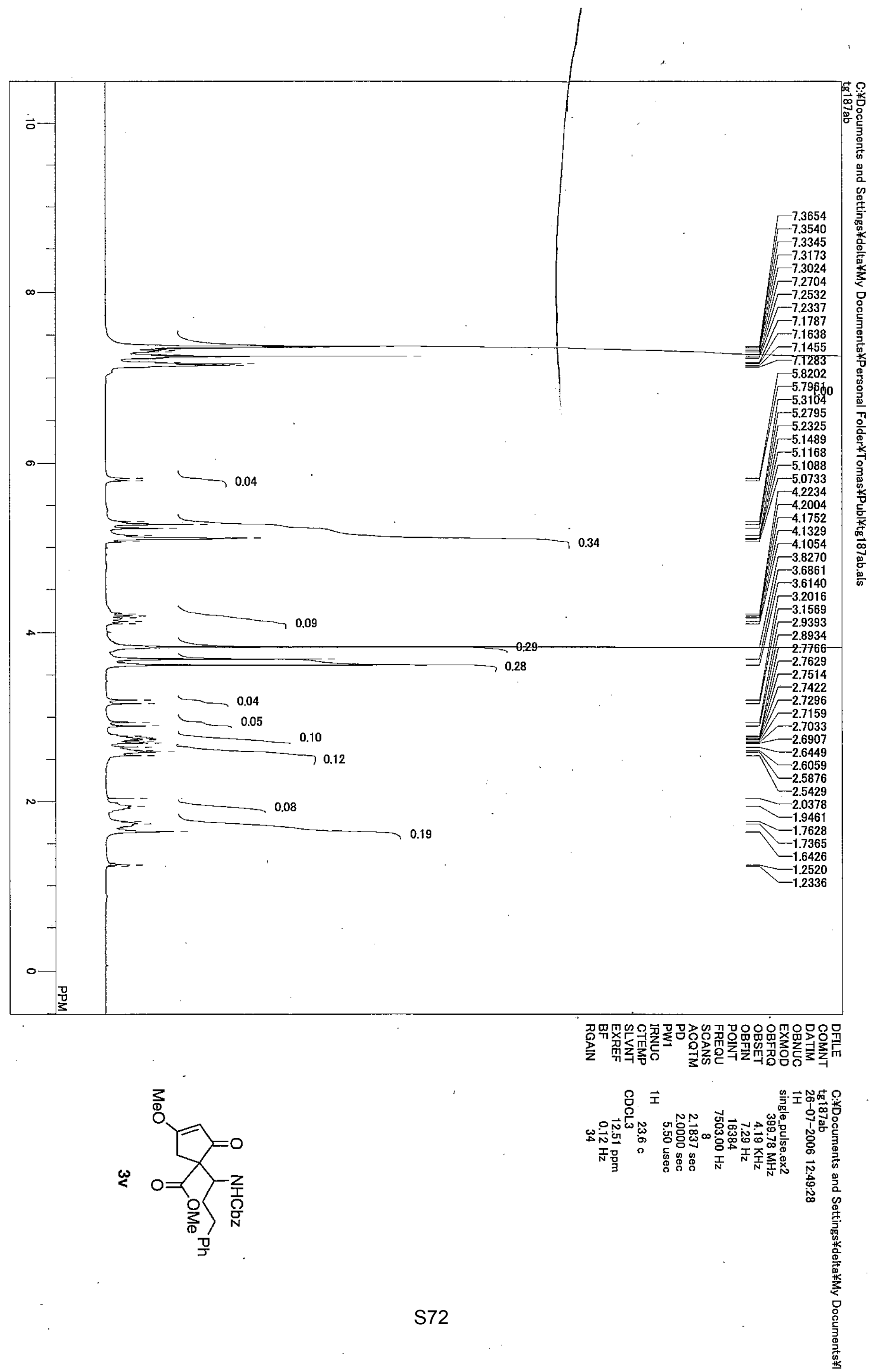




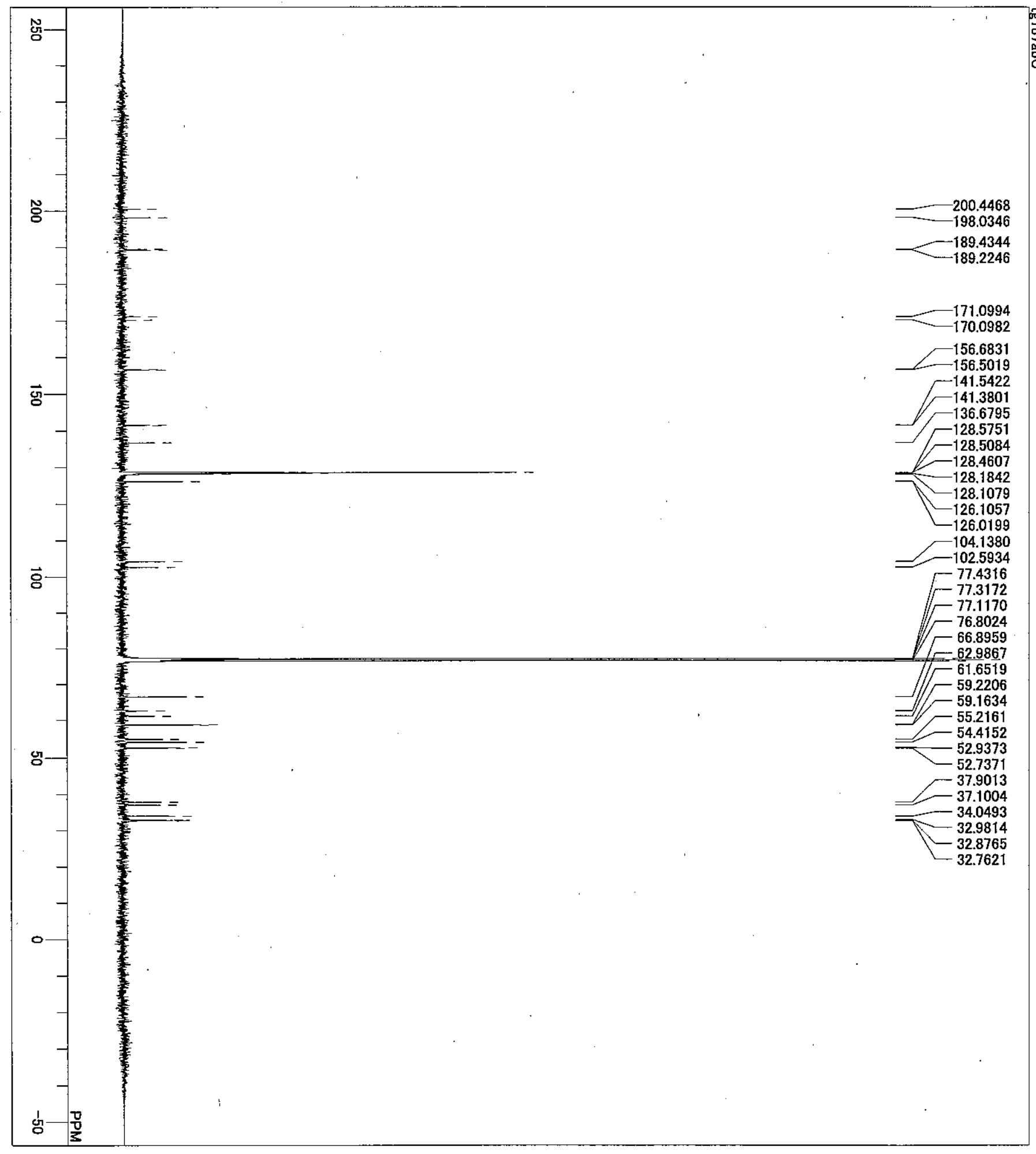

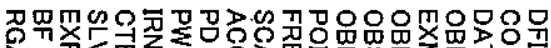

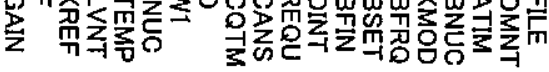<smiles>COC1=CC(=O)C(C(C)C#N)(C(C#N)C(=O)O)C1</smiles>

官 $\vec{x}$

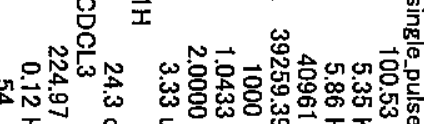

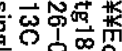
. , 


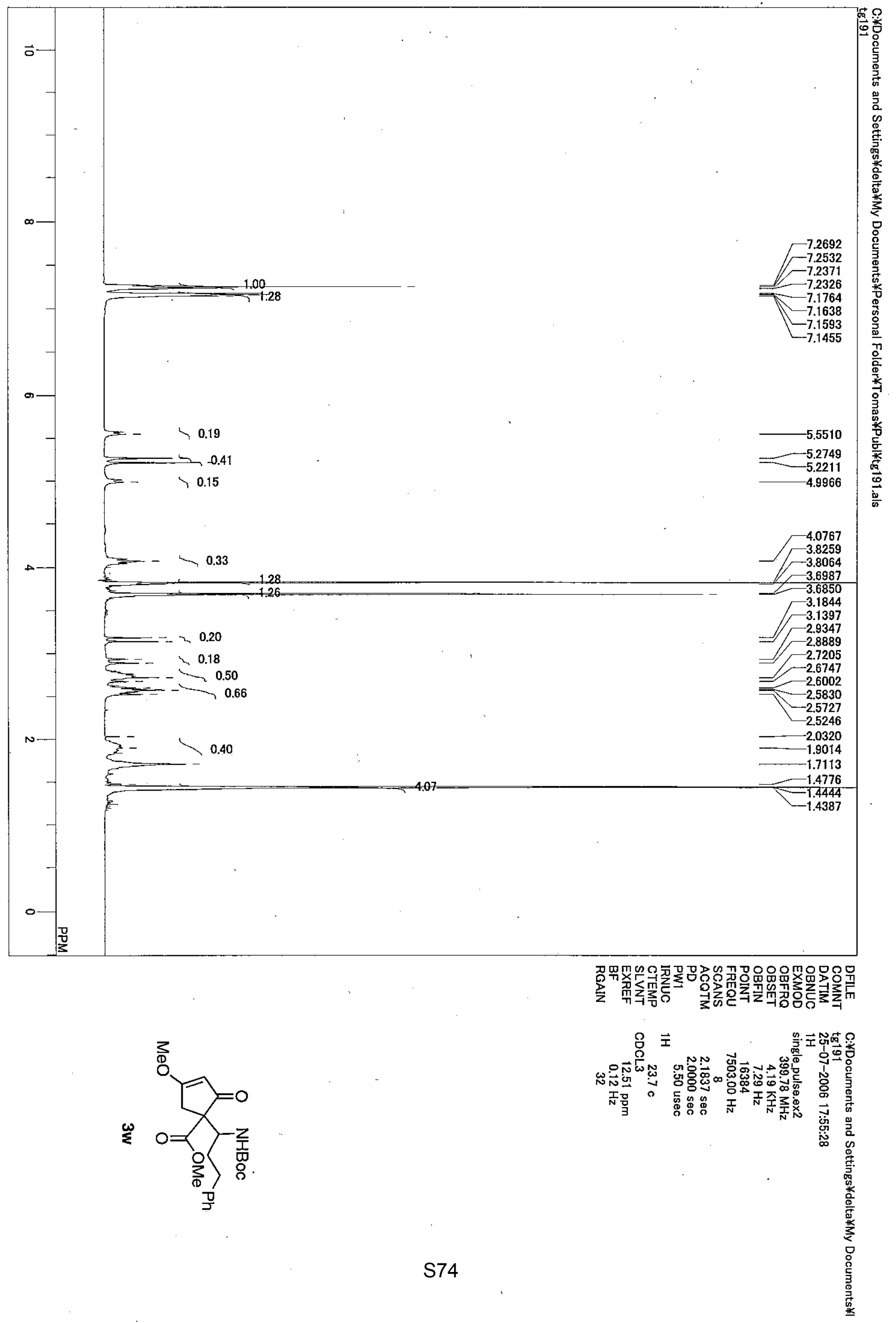




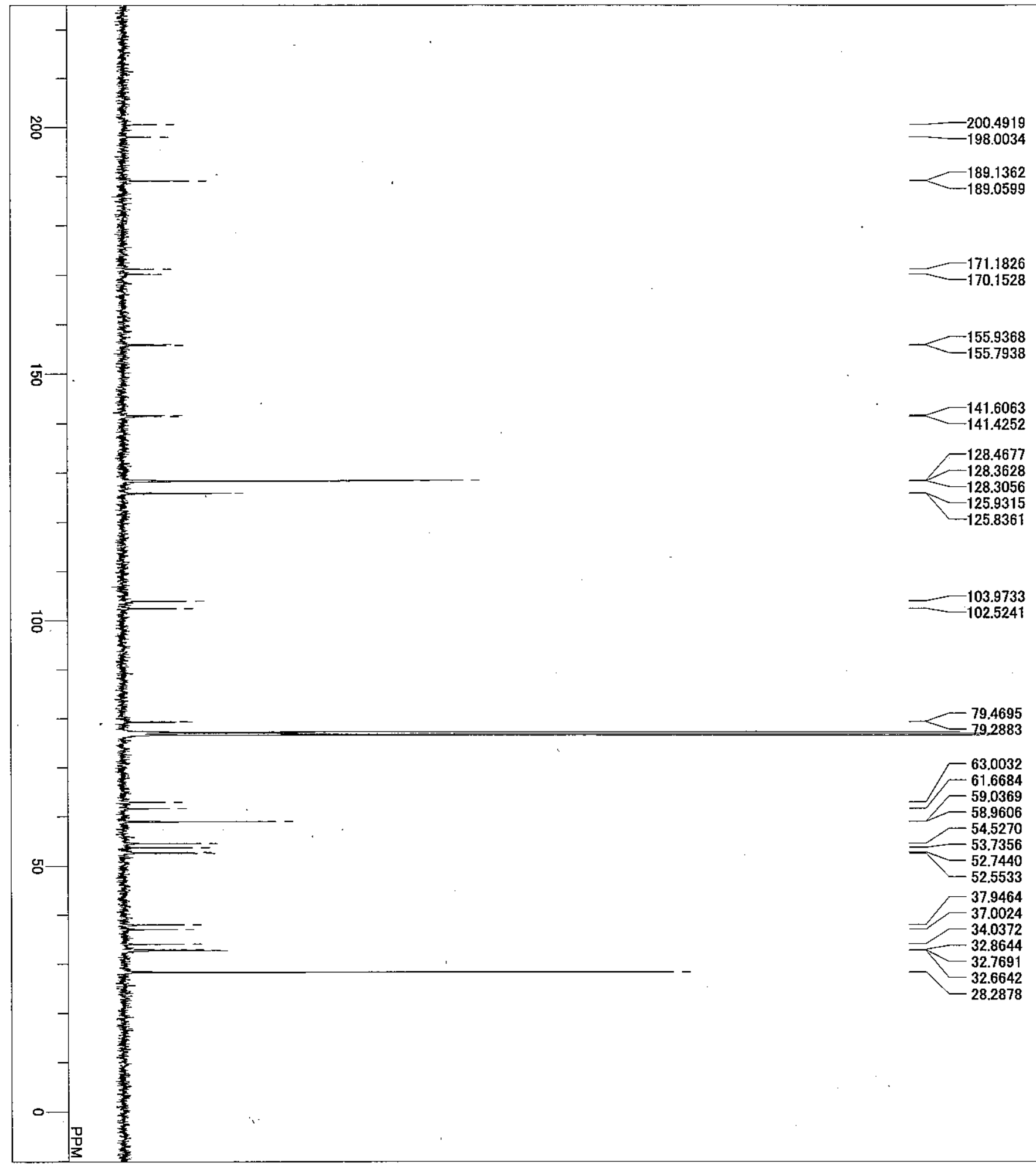

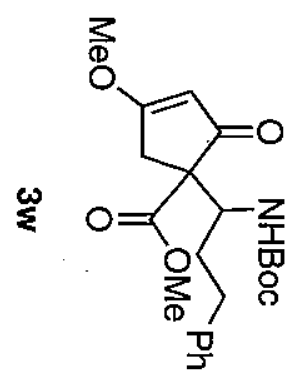




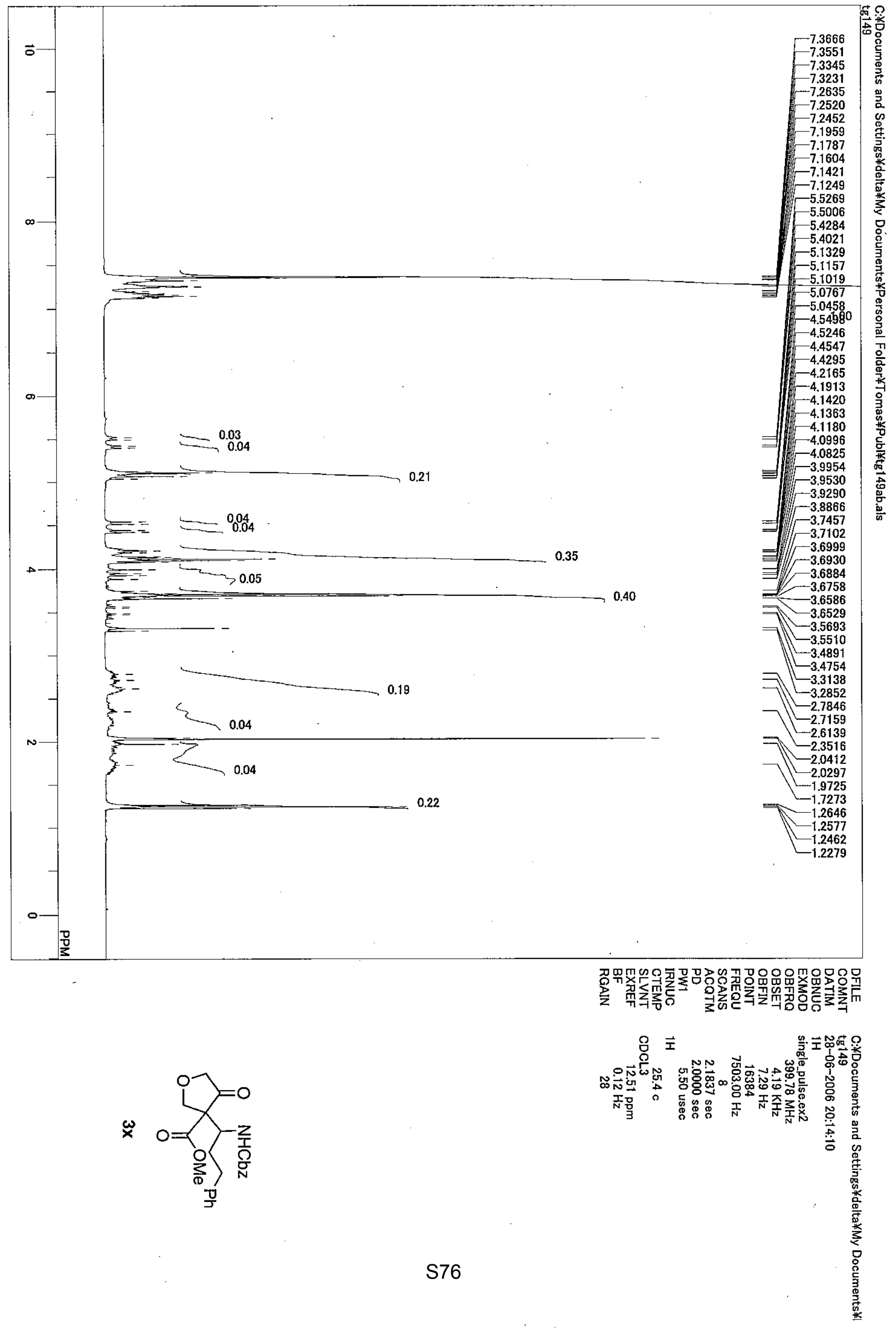




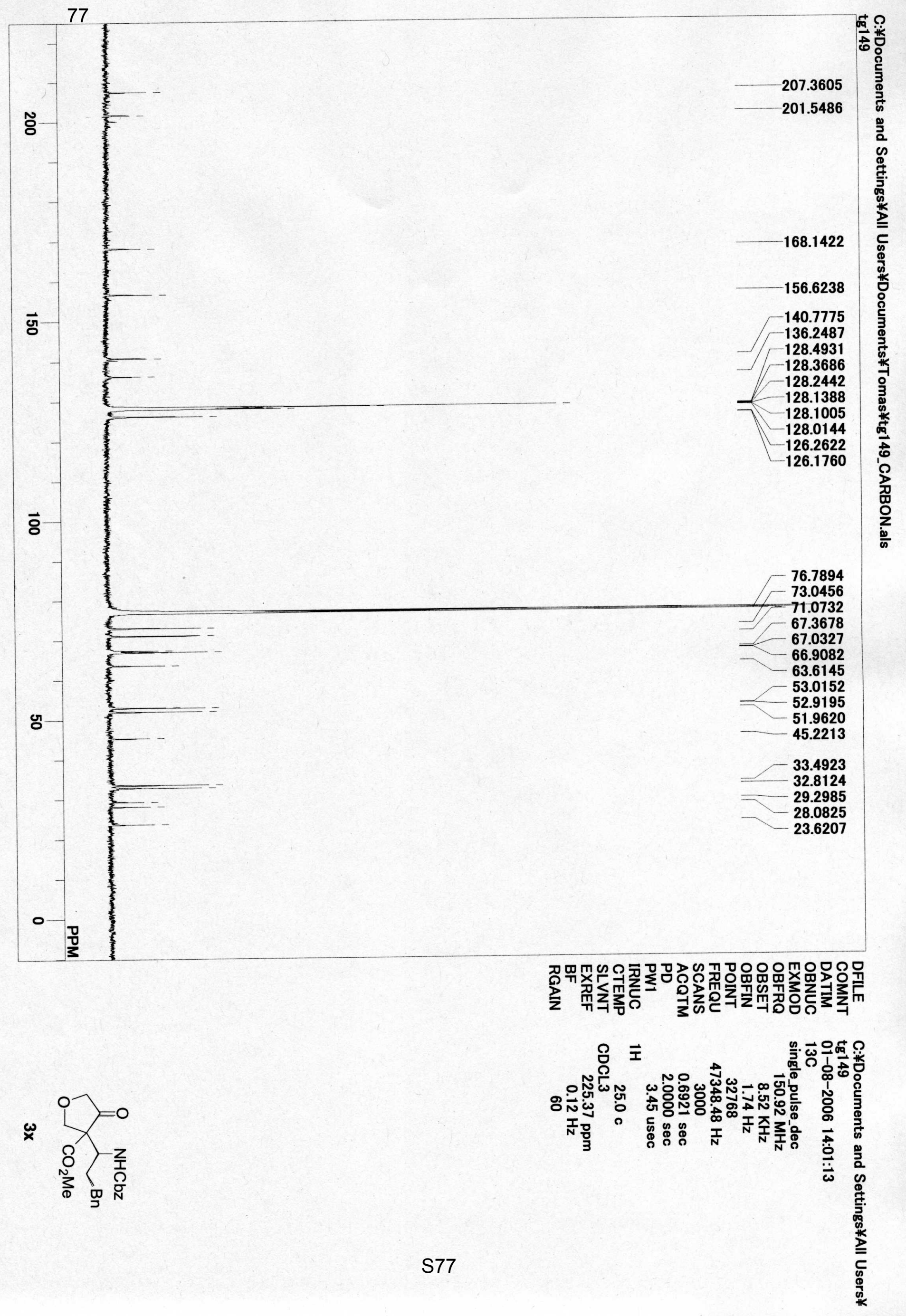




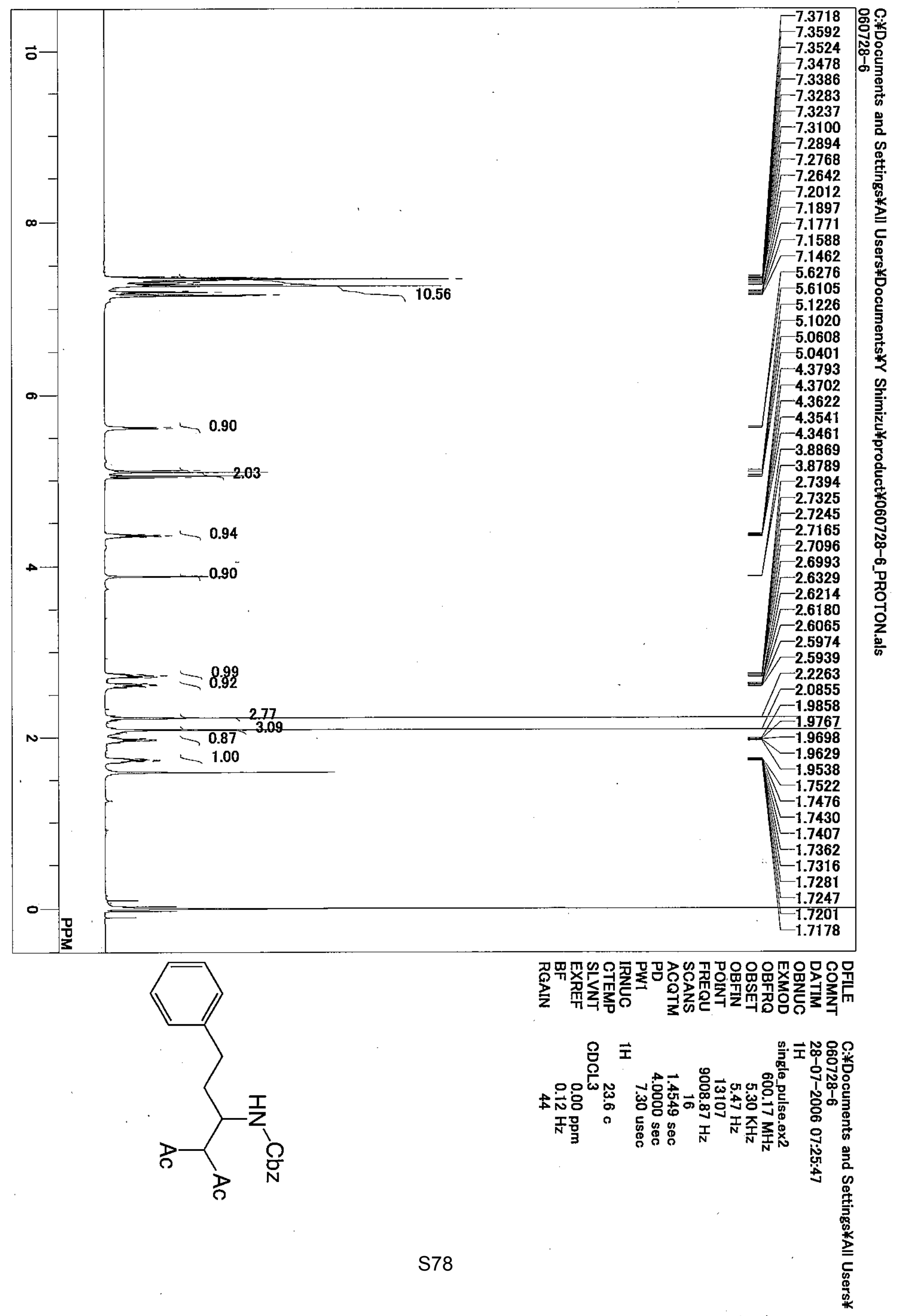




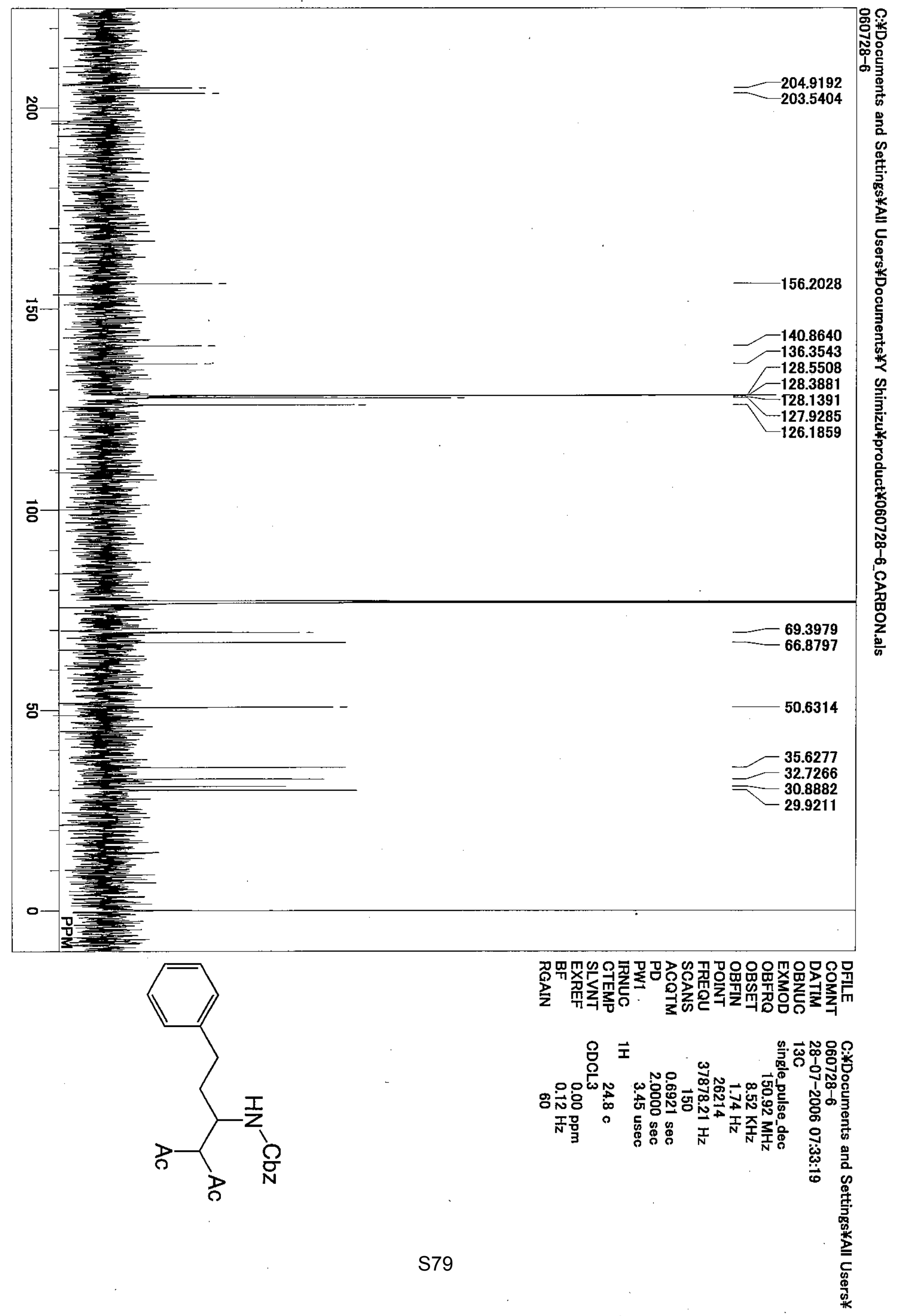




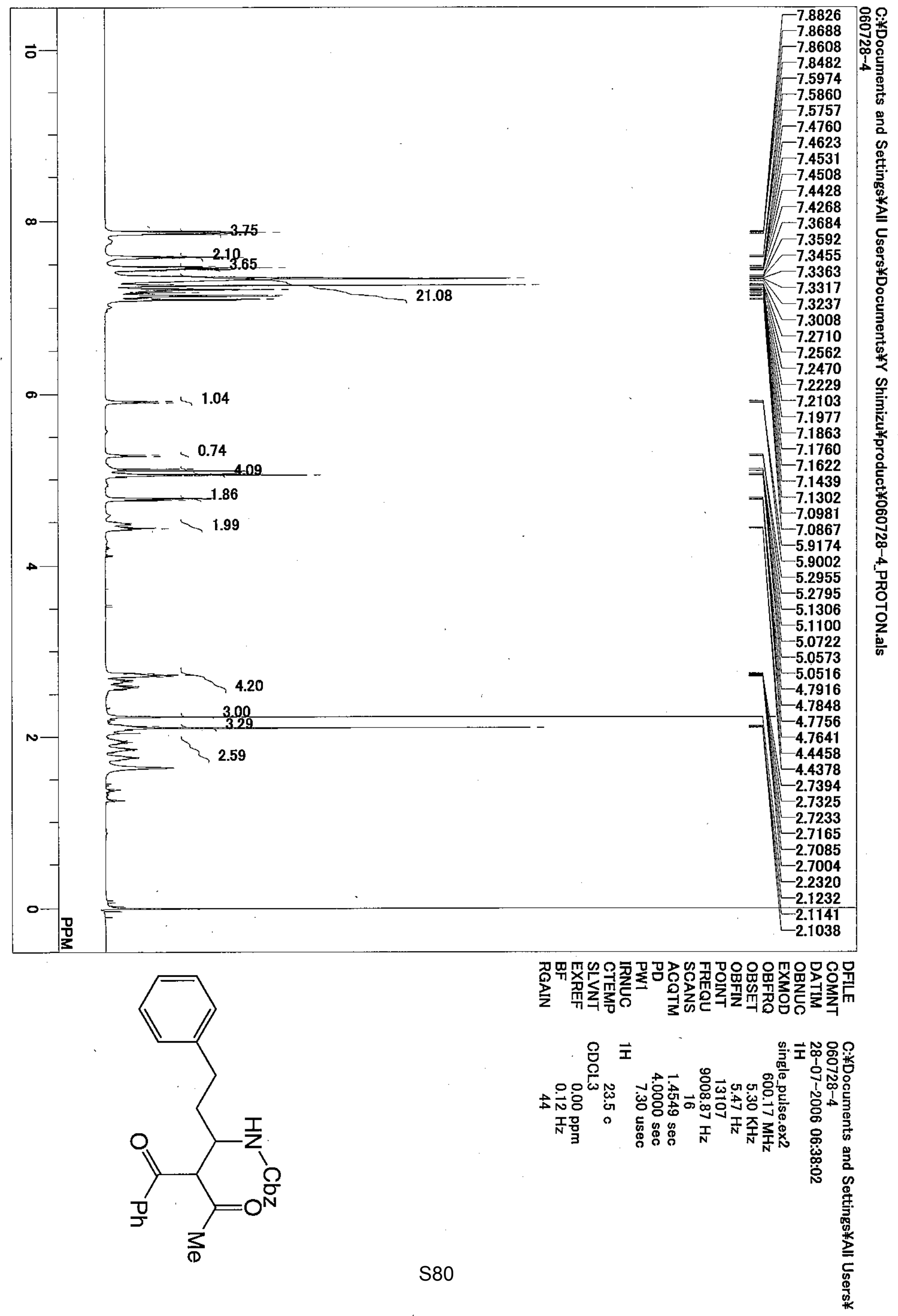




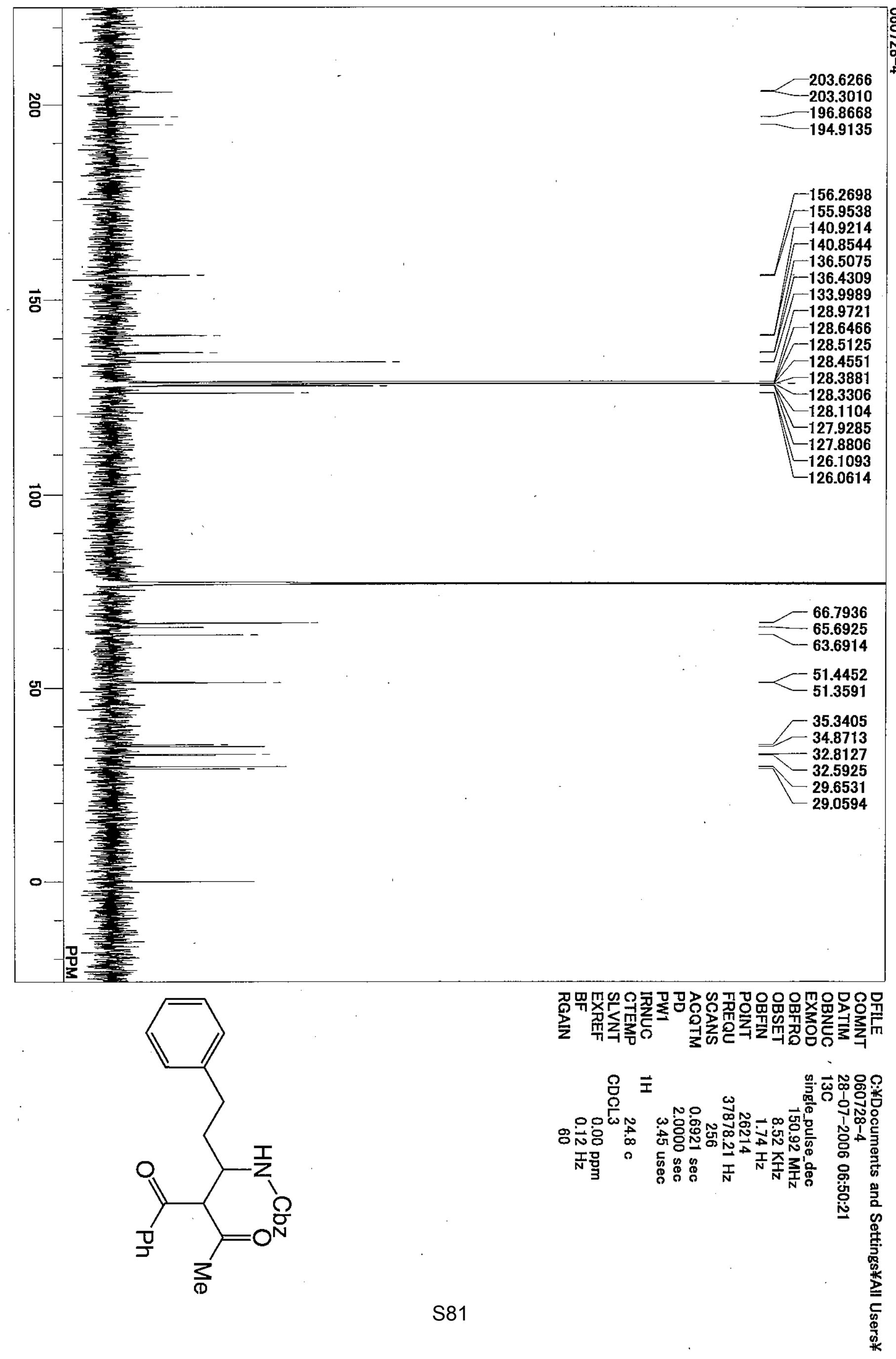

